\title{
BEREZIN QUANTIZATION AND REPRODUCING KERNELS ON COMPLEX DOMAINS
}

\author{
MIROSLAV ENGLIŠ
}

\begin{abstract}
Let $\Omega$ be a non-compact complex manifold of dimension $n, \omega=$ $\partial \bar{\partial} \Psi$ a Kähler form on $\Omega$, and $K_{\alpha}(x, \bar{y})$ the reproducing kernel for the Bergman space $A_{\alpha}^{2}$ of all analytic functions on $\Omega$ square-integrable against the measure $e^{-\alpha \Psi}\left|\omega^{n}\right|$. Under the condition

$$
K_{\alpha}(x, \bar{x})=\lambda_{\alpha} e^{\alpha \Psi(x)}
$$

F. A. Berezin [Math. USSR Izvestiya 8 (1974), 1109-1163] was able to establish a quantization procedure on $(\Omega, \omega)$ which has recently attracted some interest. The only known instances when the above condition is satisfied, however, are just $\Omega=\mathbf{C}^{n}$ and $\Omega$ a bounded symmetric domain (with the euclidean and the Bergman metric, respectively). In this paper, we extend the quantization procedure to the case when the above condition is satisfied only asymptotically, in an appropriate sense, as $\alpha \rightarrow+\infty$. This makes the procedure applicable to a wide class of complex Kähler manifolds, including all planar domains with the Poincare metric (if the domain is of hyperbolic type) or the euclidean metric (in the remaining cases) and some pseudoconvex domains in $\mathbf{C}^{n}$. Along the way, we also fix two gaps in Berezin's original paper, and discuss, for $\Omega$ a domain in $\mathbf{C}^{n}$, a variant of the quantization which uses weighted Bergman spaces with respect to the Lebesgue measure instead of the Kähler-Liouville measure $\left|\omega^{n}\right|$.
\end{abstract}

\section{INTRODUCTION}

The traditional idea of quantization, going back to Weyl and von Neumann, is that of assigning to functions ("observables") on a symplectic manifold (in this case, the euclidean space $\mathbf{R}^{2 n}$ ) operators on a separable Hilbert space in such a way that certain commutation relations (amounting to Heisenberg's uncertainty principle) are satisfied. An alternative approach, proposed much later by Berezin [Ber1], Lichnerowicz, and others, is that of deformation quantization. It consists in deforming the pointwise product of functions on the manifold into a new product $*_{h}$. This deformed product depends on a small positive parameter $h$ (Planck's constant) and is required to become the usual pointwise multiplication in the limit

Received by the editors March 15, 1995.

1991 Mathematics Subject Classification. Primary 46N50, 32A07; Secondary 46E22, 32C17, 32H10, 81S99.

Key words and phrases. Kähler manifolds, quantization, Berezin transform, weighted Bergman spaces, covariant symbols of operators, reproducing (Bergman) kernels, asymptotic behaviour, pseudoconvex domains, complex ellipsoids, Kähler-Einstein metric. 
$h \rightarrow 0$, and to satisfy the correspondence principle

$$
\lim _{h \rightarrow 0+} \frac{1}{h}\left(f *_{h} g-g *_{h} f\right)=\frac{1}{i}\{f, g\}
$$

where $\{\cdot, \cdot\}$ is the Poisson bracket. This approach, as well as various variants thereof, has recently attracted the attention of both mathematicians and physicists ([KL], [BLU], [C1], [C2], [CX]; [Rie1], [Rie2]; [BC1], [BC2], [BCZ]; [Mor]; [Pe1], [Pe2], [CGR]).

The deformed product on a Kähler manifold can be constructed with the aid of covariant symbols of operators. In brief, the basic idea of this construction is as follows. Let $\Omega$ be the manifold in question, $\omega=\sum g_{j \bar{k}} d x_{j} \wedge d \bar{x}_{k}$ the Kähler form, $i^{n} \omega^{n}$ the corresponding volume element on $\Omega$, and $\Psi$ a Kähler potential on $\Omega$, i.e. a real-valued function satisfying

$$
g_{j \bar{k}}=\frac{\partial^{2} \Psi}{\partial z_{j} \partial \bar{z}_{k}} .
$$

For $\alpha=1 / h>0$ ( $h$ is the Planck constant), we denote by $A_{\alpha}^{2}$ the Bergman space of all holomorphic functions on $\Omega$ square-integrable with respect to the measure $e^{-\alpha \Psi}(i / 2 \pi)^{n} \omega^{n} / n$ !. Let $K_{\alpha}(x, \bar{y})$ be the reproducing kernel for $A_{\alpha}^{2}$. For $T$ a bounded linear operator on $A_{\alpha}^{2}$, its covariant symbol $\widetilde{T}$ is the function on $\Omega$ given by

$$
\widetilde{T}(x)=\left\langle T K_{\alpha}(\cdot, \bar{x}), K_{\alpha}(\cdot, \bar{x})\right\rangle_{A_{\alpha}^{2}} / K_{\alpha}(x, \bar{x}) .
$$

Since the correspondence $T \mapsto \widetilde{T}$ is one-to-one, we can define multiplication of covariant symbols by

$$
\widetilde{T}_{1} *_{h} \widetilde{T}_{2}=\left(T_{1} T_{2}\right)^{\sim} \text {. }
$$

This is the required deformed product. To discuss the relation (1.1), we introduce one more definition. The Berezin transform $B_{\alpha}$ is the integral operator on $\Omega$ given by

$$
B_{\alpha} f(y)=\int_{\Omega} f(x) \frac{\left|K_{\alpha}(x, \bar{y})\right|^{2}}{K_{\alpha}(y, \bar{y})} e^{-\alpha \Psi(x)} \frac{i^{n} \omega(x)^{n}}{(2 \pi)^{n} n !} .
$$

The integral is easily seen to exist, for instance, when $f$ is a bounded measurable function on $\Omega$. Now it can be shown that the validity of the correspondence principle (1.1) is a consequence of the following asymptotic formula for the Berezin transform:

$$
B_{\alpha} f=f+\alpha^{-1} \Delta f+o\left(\alpha^{-1}\right) \quad \text { as } \alpha \rightarrow+\infty \text { (i.e. } h \rightarrow 0+\text { ). }
$$

Here $\Delta$ is the Laplace-Beltrami operator corresponding to the metric $\sum g_{j \bar{k}} d x_{j} d \bar{x}_{k}$.

Although the formula (1.2) plays a key role in Berezin's quantization procedure, he was able to prove it only under four technical assumptions. Slightly reformulated, they read as follows. (The assumption (E) was used in [Ber1] without being stated explicitly, so we have added it here.)

(E) The function $\Psi$ extends to a sesquianalytic ${ }^{1}$ function $\Psi(x, \bar{y})$ on a neighbourhood of the diagonal $\{(x, y) \in \Omega \times \Omega: x=y\}$ in $\Omega \times \Omega$ such that $\Psi(x, \bar{x})=\Psi(x)$, and the function

$$
\Psi(x, \bar{y})+\Psi(y, \bar{x})-\Psi(x, \bar{x})-\Psi(y, \bar{y})
$$

\footnotetext{
${ }^{1}$ I.e., analytic in $x$ and conjugate analytic in $y$.
} 
has a (single-valued) real analytic extension to the whole of $\Omega \times \Omega$.

(A) There is a subset $E \subset \mathbf{R}^{+}$which has $+\infty$ in its closure and is such that

$$
K_{\alpha}(z, \bar{z})=\lambda_{\alpha} \cdot e^{\alpha \Psi(z)}
$$

for each $\alpha \in E$ and suitable constants $\lambda_{\alpha}$.

(B) For all sufficiently large $\alpha \in E$ (say, $\alpha \geq \alpha_{0} \in E$ ), the functions in $A_{\alpha}^{2}$ separate the points of $\Omega$.

(C) If $z_{n}$ is a sequence in $\Omega$ and $\lim f\left(z_{n}\right)$ exists for any $f \in A_{\alpha_{0}}^{2}$, then $z_{n}$ converges to some point $z_{0} \in \Omega$.

(D) There exists $y_{0} \in \Omega$ such that $\Psi\left(\cdot, \bar{y}_{0}\right) \equiv$ const.

The assumption (D) is actually only a normalization condition. (B) and (C) are fulfilled, for instance, when the coordinate functions belong to $A_{\alpha}^{2}$ for all sufficiently large $\alpha$, and they seem to fail only in some more or less degenerate situations (cf. Examples 3.29 and 3.30).

On the other hand, the assumption (A) is very restrictive. It is satisfied for $\Omega=\mathbf{C}^{n}$ and for $\Omega$ a bounded symmetric domain, and these were the only examples where Berezin found his theory directly applicable. Although we will encounter other domains where (A) holds (cf. Example 3.30 and Remark 5.7), it seems that the equality (1.3) is indeed a rather rare phenomenon.

For this reason, it would be desirable to have the assumption (A) relaxed somewhat, and to furnish other examples where Berezin's quantization procedure can be carried out. This was first done by Peetre [Pe2] and Peetre and Engliš [EP] for the annulus with the Poincaré metric, and by the present author [E3] for $\Omega$ an arbitrary planar domain of hyperbolic type with the Poincaré metric. The main ingredient in [E3] was that (rephrased in our present terminology)

$$
\frac{\left|K_{\alpha}(x, \bar{y}) \cdot e^{-\alpha \Psi(x, \bar{y})}\right|}{2 \alpha-1}=1+O\left(\gamma^{\alpha}\right) \quad \text { as } \alpha \rightarrow+\infty, \alpha \in \mathbf{N}
$$

with $0<\gamma<1$. Thus, (A) is satisfied "asymptotically" (with $\lambda_{\alpha}=2 \alpha-1$ ); this asymptotic estimate proves sufficient for establishing (1.2).

It turns out that although (A) is rarely fulfilled, this "asymptotic version" of it holds much more often. The aim of this paper is to present a slight improvement upon Berezin's original method which makes it applicable also in this "asymptotic" case, and then to bring forth some examples demonstrating its range of applicability.

The main procedure is described in Section 2, together with minor rectifications of the exposition in [Ber1]. Owing to the latter, it turns out that the assumption (D) is unnecessary, and (B) and (C) can be replaced by a single assumption (regularity of points of $\Omega$ ) which seems better suited for practical applications. In Section 3 we treat the special case of pseudoconvex Reinhardt domains in $\mathbf{C}^{2}$ (which can be equipped with a very natural Kähler metric) in order to present some examples, such as the complex ellipsoids ${ }^{2}$ and the Springer domain; also mentioned (briefly) in a separate Appendix to this section are some examples for which the Kähler metric degenerates at some points or has singularities. Section 4 discusses a slight (though possibly less natural from the point of view of Riemannian geometry) variation of the original quantization procedure, when the Bergman spaces $A_{\alpha}^{2}$ are taken

\footnotetext{
${ }^{2}$ The (somewhat less dignified) term "eggs" has been suggested by S. Krantz [Krn, p. 126].
} 
with respect to the Lebesgue measure instead of the Liouville volume $i^{n} \omega^{n}$. Some final comments (on holomorphic invariance, Kähler-Einstein metrics, curvature, negligible sets, etc.) and open problems are collected in the last Section 5.

We shall often deal with functions of two variables which are analytic in the first variable and conjugate-analytic in the second (sesquianalytic functions; sesquimeromorphic functions are defined similarly). Such functions will be denoted as $f(x, \bar{y})$, and their restriction to the diagonal (which is a real-analytic function of one variable) as $f(x, \bar{x})$.

\section{QuANTIZATION ON KÄHLER MANIFOLDS}

Let $\Omega$ be a Kähler manifold, with the Kähler form $\omega$ and the corresponding Poisson bracket $\{\cdot, \cdot\}$. By a (special) quantization of $(\Omega, \omega)$, we understand an associative algebra $\mathcal{A}$ with involution which satisfies the following:

(1) There is a family $\mathcal{A}_{h}$ of associative algebras with involution such that

(1a) each $\mathcal{A}_{h}$ is an algebra of functions on $\Omega$, with pointwise addition and scalar multiplication, and with complex conjugation as the involution;

(1b) the index $h$ runs through a set $E \subset(0,+\infty)$ which has 0 in its closure;

(1c) $\mathcal{A}$ is a subalgebra of the direct sum $\bigoplus\left\{\mathcal{A}_{h}, h \in E\right\}$.

The products in $\mathcal{A}_{h}$ and $\mathcal{A}$ will be denoted by $*_{h}$ and $*$, respectively, and elements of $\mathcal{A}$ will be written as $f(h \mid x)(h \in E, x \in \Omega)$, so that $f(h \mid \cdot) \in \mathcal{A}_{h}$.

(2) For each $f \in \mathcal{A}$ and $x \in \Omega$, the limit

$$
\lim _{h \rightarrow 0+} f(h \mid x)=\varphi(f)(x)
$$

exists, and the mapping $\varphi$ has the following properties. There exists a linear manifold $\widetilde{\mathcal{A}} \subset \mathcal{A}$ such that

(2a) $\varphi\left(f_{1} * f_{2}\right)=\varphi\left(f_{1}\right) \cdot \varphi\left(f_{2}\right)$ when $f_{1}, f_{2} \in \widetilde{\mathcal{A}}$;

(2b) $\varphi\left(\frac{1}{h}\left(f_{1} * f_{2}-f_{2} * f_{1}\right)\right)=\frac{1}{i}\left\{\varphi\left(f_{1}\right), \varphi\left(f_{2}\right)\right\}$ when $f_{1}, f_{2} \in \widetilde{\mathcal{A}}$;

(2c) for any pair of points $x_{1}, x_{2} \in \Omega$ there exists $f \in \widetilde{\mathcal{A}}$ such that $\varphi(f)\left(x_{1}\right) \neq$ $\varphi(f)\left(x_{2}\right)$.

The parameter $h$ plays the role of Planck's constant. The conditions (2a) and (2b) are termed the (weak) correspondence principle [Ber1]. (The strong correspondence principle is obtained upon requiring that $\widetilde{\mathcal{A}}=\mathcal{A}$.) (2c) assures that there are sufficiently many "quantizable" functions on $\Omega$ (i.e. functions in the range of $\varphi$ ). No topological or continuity assumptions are laid on $\mathcal{A}_{h}$ and $\varphi$.

As was sketched in the Introduction, the algebras $\mathcal{A}_{h}$ will be constructed with the aid of covariant symbols of operators. We recall some basic general facts. Let $\Omega$ be a complex manifold, $\mu$ a measure on $\Omega$, and $\mathcal{H}$ a subspace of $L^{2}(\Omega, \mu)$ whose elements are functions analytic on $\Omega$ and which admits a reproducing kernel $K$. $K=K(x, \bar{y})$ is then a function analytic in the first variable and antianalytic in the second. For a bounded linear operator $A$ on $\mathcal{H}$, we have

$$
\begin{aligned}
A f(x)=\langle A f, K(\cdot, \bar{x})\rangle & =\left\langle f, A^{*} K(\cdot, \bar{x})\right\rangle \\
& =\int_{\Omega} f(y) \overline{\left(A^{*} K(\cdot, \bar{x})\right)(y)} d \mu(y)=\int_{\Omega} f(y) K_{A}(x, \bar{y}) d \mu(y),
\end{aligned}
$$

where

$$
K_{A}(x, \bar{y})=\overline{\left(A^{*} K(\cdot, \bar{x})\right)(y)}=\left\langle K(\cdot, \bar{y}), A^{*} K(\cdot, \bar{x})\right\rangle=\langle A K(\cdot, \bar{y}), K(\cdot, \bar{x})\rangle .
$$


Thus, each bounded linear operator $A$ on $\mathcal{H}$ is an integral operator with kernel $K_{A}$. An easy calculation shows that $K_{A+B}=K_{A}+K_{B}, K_{c A}=c K_{A}$ for $c \in \mathbf{C}$, $K_{A^{*}}(x, \bar{y})=\overline{K_{A}(y, \bar{x})}$,

$$
K_{I}(x, \bar{y})=K(x, \bar{y})
$$

where $I$ is the identity operator on $\mathcal{H}$, and

$$
K_{A B}(x, \bar{y})=\int_{\Omega} K_{A}(x, \bar{z}) K_{B}(z, \bar{y}) d \mu(z) .
$$

Of special interest for us will be the function

$$
\widetilde{A}(x, \bar{y})=\frac{K_{A}(x, \bar{y})}{K(x, \bar{y})} .
$$

This is a meromorphic function of $x$, conjugate-meromorphic of $y$, and satisfies

$$
\begin{aligned}
\widetilde{I}(x, \bar{y})=\mathbf{1}, \quad(A+B)^{\sim} & =\widetilde{A}+\widetilde{B}, \quad \widetilde{c A}=c \widetilde{A} \quad(c \in \mathbf{C}), \quad \widetilde{A^{*}}(x, \bar{y})=\overline{\widetilde{A}(y, \bar{x}),} \\
\widetilde{A B}(x, \bar{y}) & =\int_{\Omega} \widetilde{A}(x, \bar{z}) \widetilde{B}(z, \bar{y}) \frac{K(x, \bar{z}) K(z, \bar{y})}{K(x, \bar{y})} d \mu(z),
\end{aligned}
$$

and

$$
A f(x)=\int_{\Omega} \widetilde{A}(x, \bar{y}) K(x, \bar{y}) f(y) d \mu(y) .
$$

The restriction of $\widetilde{A}(x, \bar{y})$ to the diagonal,

$$
\widetilde{A}(x)=\widetilde{A}(x, \bar{x}), \quad x \in \Omega,
$$

is called the covariant symbol of the operator $A$. In view of the Schwarz inequality,

$$
|\widetilde{A}(x, \bar{x})| \leq\|A\| \quad \forall x \in \Omega,
$$

so $\widetilde{A}(x)$ is a bounded real-analytic function on $\Omega$. Denote

$$
\mathcal{A}=\{\widetilde{A}(x): A \text { a bounded linear operator on } \mathcal{H}\}
$$

Since an analytic function of two variables $x, y$ is uniquely determined by its restriction to the anti-diagonal $x=\bar{y}$ (cf. [BM], Proposition II.4.7, or [E1], Proposition 1, for instance), it follows that $\widetilde{A}(x)$ uniquely determines $\widetilde{A}(x, \bar{y})$ and, hence, also $K_{A}(x, \bar{y})$ and $A$; in other words, the correspondence $A \leftrightarrow \widetilde{A}(x)$ is one-to-one. Thus we can define multiplication in $\mathcal{A}$ by

$$
(\widetilde{A} * \widetilde{B})(x)=\widetilde{A B}(x),
$$

addition and scalar multiplication by $\widetilde{A}+\widetilde{B}=(A+B)^{\sim}, c \widetilde{A}=(c A)^{\sim}$ (these coincide with the usual pointwise operations), and involution by $(\widetilde{A})^{*}=\widetilde{A^{*}}$ (which coincides with the complex conjugation). This makes $\mathcal{A}$ into an (associative, but 
not commutative) *-algebra of bounded real-analytic functions on $\Omega$, with identity element $\widetilde{I}=\mathbf{1}$. $\mathcal{A}$ is called the algebra of covariant symbols for $\mathcal{H}$. It is isomorphic to the algebra of all bounded linear operators on $\mathcal{H}$.

Let us now come back to our Kähler manifold $\Omega$, with the Kähler form

$$
\omega=\sum_{j, k=1}^{n} g_{j \bar{k}} d x_{j} \wedge d \bar{x}_{k} .
$$

According to the definition of a Kähler manifold, we have $d \omega=0$ and $g=$ $\operatorname{det}\left(g_{j \bar{k}}\right)_{j, k=1}^{n} \neq 0$. A Kähler potential is a function $\Psi$ on $\Omega$ such that

$$
\frac{\partial^{2} \Psi}{\partial z_{j} \partial \bar{z}_{k}}=g_{j \bar{k}} .
$$

The Kähler condition $d \omega=0$ is also a necessary and sufficient condition for existence of local solutions to $(2.9)$. If $\Omega$ is simply connected, ${ }^{3}$ these can be glued into a global solution. If $\Omega$ is not simply connected, then a global solution exists at least on an open set $\widetilde{\Omega}$ obtained from $\Omega$ by deleting a (real) submanifold of lower (real) dimension - this is the point of view employed in Berezin's later works [Ber2]. Alternatively, one may pass to the universal cover of $\Omega$ to get a global solution; we shall demonstrate this in Examples 2.15 and 2.16 below.

Since the matrix $g_{j \bar{k}}$ is Hermitian, $\bar{\Psi}$ and $\frac{\Psi+\bar{\Psi}}{2}$ are solutions of (2.9) whenever $\Psi$ is. Thus, a solution of (2.9) can always be chosen to be real-valued, and we will henceforth assume that this is so.

To the form $\omega$ corresponds the Riemannian metric

$$
d s^{2}=\sum_{j, k=1}^{n} g_{j \bar{k}} d x_{j} d \bar{x}_{k} .
$$

Let

$$
\begin{aligned}
d \mu(z)=\frac{i^{n} \omega^{n}}{(2 \pi)^{n} n !} & =g(z) \prod_{j=1}^{n} \frac{i d z_{j} \wedge d \bar{z}_{j}}{2 \pi} \\
& =g(z) \prod_{j=1}^{n} \frac{d x_{j} \wedge d y_{j}}{\pi} \quad\left(z_{j}=x_{j}+i y_{j}\right)
\end{aligned}
$$

be the corresponding volume element on $\Omega$ (normalized by the factor $\pi^{-n}$ ). Here, as above,

$$
g=\operatorname{det}\left(g_{j \bar{k}}\right) \neq 0 .
$$

For $\alpha>0$, denote

$$
\|f\|_{\alpha}^{2}=\int_{\Omega}|f(x)|^{2} e^{-\alpha \Psi(x)} d \mu(x)
$$

\footnotetext{
${ }^{3}$ In the sense that every closed path in $\Omega$ is homotopic to a constant path.
} 
and let $A_{\alpha}^{2}$ be the Bergman space of all analytic functions on $\Omega$ for which $\|f\|_{\alpha}<$ $+\infty$. (It may happen that $A_{\alpha}^{2}$ contains only the constant zero.) Since both $g$ and $\Psi$ are continuous functions on $\Omega$ and $g$ does not vanish, it follows easily from the mean value property of analytic functions that

$$
|f(x)| \leq C(\alpha, x) \cdot\|f\|_{\alpha} \quad \forall f \text { analytic on } \Omega
$$

for some constants $C(\alpha, x)<+\infty$. By a standard argument [Helg, Section VIII.3], this implies that $A_{\alpha}^{2}$ is a closed subspace of $L^{2}\left(\Omega, e^{-\alpha \Psi} d \mu\right)$ (hence, a Hilbert space) and that it admits a reproducing kernel, which we will denote $K_{\alpha}(x, \bar{y})$. Of course, if $A_{\alpha}^{2}$ reduces to the constant zero, then $K_{\alpha}(x, \bar{y}) \equiv 0$ identically.

The basic tool for Berezin's quantization are the algebras of covariant symbols for $A_{\alpha}^{2}$. Note that for $\mathcal{H}=A_{\alpha}^{2}$ and $x=y$, the formulas (2.3) and (2.7) give

$$
\widetilde{A} * \widetilde{B}(x)=\int_{\Omega} \widetilde{A}(x, \bar{z}) \widetilde{B}(z, \bar{x}) \frac{\left|K_{\alpha}(x, \bar{z})\right|^{2}}{K_{\alpha}(x, \bar{x})} e^{-\alpha \Psi(z)} d \mu(z) .
$$

As we have already remarked in the Introduction, the integral operator

$$
B_{\alpha} f(x)=\int_{\Omega} f(z) \frac{\left|K_{\alpha}(x, \bar{z})\right|^{2}}{K_{\alpha}(x, \bar{x})} e^{-\alpha \Psi(z)} d \mu(z)
$$

is called the Berezin transform on $\Omega$ and will play a prominent rôle in this paper.

We will gradually introduce (just as Berezin did) a number of assumptions concerning $\Psi$ and $K_{\alpha}$ which are needed for going further, and supply some comments on them. Their labelling, $\left(\mathrm{A}^{\prime}\right),\left(\mathrm{C}^{\prime}\right)$ and $\left(\mathrm{E}^{\prime}\right)$, corresponds to the labelling of Berezin's original assumptions in the Introduction and (with the exception of (E)) in [Ber1]. For reasons of exposition, they will be revealed in the order $\left(\mathrm{E}^{\prime}\right),\left(\mathrm{A}^{\prime}\right)$ and $\left(\mathrm{C}^{\prime}\right)$, and followed by an explanation of why there is no $\left(\mathrm{D}^{\prime}\right)$ and $\left(\mathrm{B}^{\prime}\right)$.

Assumption $\left(\mathrm{E}^{\prime}\right)$. There exists $\alpha_{0}>0$ such that the function

$$
a(x, \bar{x})=e^{-\alpha_{0} \Psi(x)}
$$

extends to a sesquimeromorphic function $a(x, \bar{y})$ on $\Omega \times \Omega$.

By changing the scale (i.e. replacing $\alpha$ by $\alpha / \alpha_{0}$ ), we can assume, without loss of generality, that $\alpha_{0}=1$, and we will do so from now on. Thus, $a(x, \bar{x})=e^{-\Psi(x)}$.

Recall that $\Psi$ was defined as a (real-valued) solution of (2.9). If the functions $g_{j \bar{k}}$ are restrictions to the diagonal $y=x$ of some sesquianalytic functions $g_{j \bar{k}}(x, \bar{y})$ on $\Omega \times \Omega$, then there locally exist solutions also to the system of equations

$$
\frac{\partial^{2} \Psi(x, \bar{y})}{\partial x_{j} \partial \bar{y}_{k}}=g_{j \bar{k}}(x, \bar{y})
$$

If the local solutions can be glued into a global sesquianalytic function $\Psi(x, \bar{y})$, then $\left(\mathrm{E}^{\prime}\right)$ is satisfied for any $\alpha_{0}>0$, with $a(x, \bar{y})=e^{-\alpha_{0} \Psi(x, \bar{y})}$. In general, the glueing process will produce a multiple-valued function $\Psi$, but it can still happen that all the "periods" of $\Psi$ are integral multiples of a fixed purely imaginary number (say, $c i, c>0$ ); then the choice $\alpha_{0}=2 \pi / c$ does the job. In all other cases, it is necessary 
to modify the whole approach a little by first passing to the universal cover of $\Omega$; see Examples 2.15 and 2.16 below.

The corresponding counterpart of $\left(\mathrm{E}^{\prime}\right)$ in Berezin's assumptions (which is not explicitly labelled in [Ber1], so we have christened it (E) in our Introduction) seems, at a first glance, a little weaker. However, in all situations where Berezin was able to apply it directly, $\Psi(x)$ was the logarithm of the restriction to the diagonal of a sesquianalytic function, i.e. $\Psi(x, \bar{y})$ was a multiple-valued sesquianalytic function with the same "periods" as the logarithm, ${ }^{4}$ that is, integer multiples of $2 \pi i$. But this is exactly the situation described in the preceding paragraph (with $c=2 \pi$ and, consequently, $\alpha_{0}=2 \pi / c=1$ ). Thus, in all cases where Berezin's (E) worked, so will our $\left(\mathrm{E}^{\prime}\right)$.

Since $a(x, \bar{y})$ is a sesquimeromorphic function which is real-valued (even positive) for $x=y$, it follows that

$$
a(x, \bar{y})=\overline{a(y, \bar{x})} .
$$

(To see this, make a substitution $x=u+i v, \bar{y}=u-i v, a(x, \bar{y})=H(u, v)$, $u, v \in \mathbf{C}^{n} . H$ is then a meromorphic function which is real for $u, v \in \mathbf{R}^{n}$; hence, its Taylor coefficients are real, and (2.16) follows. See [E1], Proposition 1, or [BM], Proposition II.4.7, for details.)

The next - and crucial - assumption concerns the behaviour of the reproducing kernels $K_{\alpha}$.

Assumption $\left(\mathrm{A}^{\prime}\right)$. There exists an infinite subset $E$ of the positive integers such that for all $\alpha \in E$ and $x, y \in \Omega$,

$$
K_{\alpha}(x, \bar{y}) a(x, \bar{y})^{\alpha}=c_{n} \alpha^{n}+B(x, \bar{y}) \alpha^{n-1}+C(x, \bar{y}, \alpha) \alpha^{n-2},
$$

where $c_{n} \in \mathbf{C}$, and $B(x, \bar{y})$ and $C(x, \bar{y}, \alpha)$ are sesquianalytic functions in $x$ and $y$ which satisfy

$$
\sup _{x, y \in \Omega}|B(x, \bar{y})|<+\infty, \quad \sup _{\substack{x, y \in \Omega, \alpha \in E}}|C(x, \bar{y}, \alpha)|<+\infty .
$$

Here $n$ is the (complex) dimension of $\Omega$.

Frequently we will be able to manage with a somewhat weaker assumption:

Assumption $\left(\mathrm{A}^{\prime \prime}\right)$. There exists an infinite subset $E$ of the positive integers such that for all $\alpha \in E$ and $x, y \in \Omega$,

$$
K_{\alpha}(x, \bar{y}) a(x, \bar{y})^{\alpha}=c_{n} \alpha^{n}+B(x, \bar{y}) \alpha^{n-1}+C(x, \bar{y}, \alpha) \alpha^{n-2}
$$

where $c_{n} \in \mathbf{C}$ and the functions $B$ and $C$ are sesquianalytic in $x$ and $y$ and satisfy

$$
\sup _{x \in \Omega}|B(x, \bar{y})|<+\infty, \quad \sup _{x \in \Omega, \alpha \in E}|C(x, \bar{y}, \alpha)|<+\infty
$$

\footnotetext{
${ }^{4}$ In fact, the domains considered in [Ber1] were even simply connected, so a single-valued branch of the logarithm exists and no "periods" are needed.
} 
for each $y \in \Omega$. Here $n$ is the (complex) dimension of $\Omega$.

The restriction that $E$ be a subset of the integers is, of course, imposed by the requirement that $a(x, \bar{y})^{\alpha}$ be well-defined. Otherwise, it's quite immaterial; we will be interested mainly in the limit as $\alpha \rightarrow+\infty$, so any unbounded subset of the positive reals would be adequate for our purposes.

The corresponding assumption (A) in [Ber1] is that

$$
K_{\alpha}(x, \bar{x})=\lambda_{\alpha} \cdot e^{\alpha \Psi(x)}
$$

for some constants $\lambda_{\alpha}$, and, somewhat later, $\lambda_{\alpha}$ are shown to behave asymptotically as $\alpha^{n}$ when $\alpha \rightarrow+\infty$. This explains the leading term $c_{n} \alpha^{n}$ (we will soon show that necessarily $\left.c_{n}=1\right)$. If $\lambda_{\alpha} \neq 0,(2.18)$ implies that $e^{\alpha \Psi(x)}$ extends to a (singlevalued) sesquianalytic function $e^{\alpha \Psi(x, \bar{y})}$ on $\Omega \times \Omega$ (although $\Psi(x, \bar{y})$ itself need not!) and

$$
K_{\alpha}(x, \bar{y})=\lambda_{\alpha} \cdot e^{\alpha \Psi(x, \bar{y})} \quad \forall x, y \in \Omega
$$

Thus we see that our $\left(\mathrm{A}^{\prime}\right)$ is, indeed, weaker than Berezin's $(\mathrm{A})$.

Note that the deduction of (2.19) from (2.18) in the last paragraph can also be done when (2.18) is replaced by the equality (2.17) for $x=y$. Thus, it would be sufficient to require only that the equality in $\left(\mathrm{A}^{\prime}\right)$ be valid for $y=x$, and similarly for $\left(\mathrm{A}^{\prime \prime}\right)$.

The behaviour of the reproducing kernels can be pretty wild. We shall give an example of a Kähler manifold $(\Omega, \omega)$ with a closed submanifold $\Omega_{0} \subsetneq \Omega$ such that

$$
K_{\alpha}(x, \bar{x}) e^{-\alpha \Psi(x)} \sim \alpha^{2} \quad \text { for all } x \in \Omega \backslash \Omega_{0}
$$

while

$$
K_{\alpha}(x, \bar{x}) e^{-\alpha \Psi(x)} \sim 2 \alpha^{2} \quad \text { for } x \in \Omega_{0}
$$

as $\alpha \rightarrow+\infty$. In fact, $\Omega$ will be a certain pseudoconvex domain in $\mathbf{C}^{2}$, and $\Omega_{0}$ its intersection with the hyperplane $x_{1}=0$. See Example 3.31.

We finally remark that even the assumption $\left(\mathrm{A}^{\prime}\right)$ is still fairly strong, and it's possible to carry out the Berezin quantization procedure even sometimes when it fails; see again Examples 2.15 and 2.16.

Note that, in view of $(2.16)$,

$$
\frac{a(x, \bar{x}) a(y, \bar{y})}{a(x, \bar{y}) a(y, \bar{x})} \geq 0 \quad \forall x, y \in \Omega
$$

Thus we may define a real-valued function $\psi$ on $\Omega \times \Omega$ by

$$
\psi(x \mid y) \equiv \psi(x, \bar{x} \mid y, \bar{y})=\log \frac{a(x, \bar{x}) a(y, \bar{y})}{a(x, \bar{y}) a(y, \bar{x})}
$$

$(\psi$ may assume the value $-\infty)$. Formally, we have, by $\left(\mathrm{E}^{\prime}\right)$ (remember that $\alpha_{0}=1$ !)

$$
\psi(x, \bar{x} \mid y, \bar{y})=\Psi(x, \bar{y})+\Psi(y, \bar{x})-\Psi(x, \bar{x})-\Psi(y, \bar{y}) .
$$


A point $y \in \Omega$ will be called regular if $\psi(\cdot \mid y) \leq 0$ and for any sequence $x_{n}$ of points in $\Omega$,

$$
\psi\left(x_{n} \mid y\right) \rightarrow 0 \quad \text { implies } \quad x_{n} \rightarrow y .
$$

This can be reformulated as follows: for each neighbourhood $U$ of $y$, there exists $\delta>0$ such that $\psi(x \mid y) \leq-\delta$ for all $x \notin U$. In other words, the function $\psi(\cdot \mid y)$ has a local maximum at $y$, which is also a global maximum and even dominates all cluster values of $\psi(\cdot \mid y)$ on the boundary of $\Omega$.

It turns out that when $\left(\mathrm{A}^{\prime \prime}\right)$ holds, the first condition in the definition of regularity is superfluous.

Proposition 2.1. Assume that $\left(\mathrm{A}^{\prime \prime}\right)$ holds. Then $\psi \leq 0$.

Proof. Fix $x, y \in \Omega$. By the Schwarz inequality,

$$
\frac{\left|K_{\alpha}(x, \bar{y})\right|^{2}}{K_{\alpha}(x, \bar{x}) K_{\alpha}(y, \bar{y})} \leq 1
$$

If $\left(\mathrm{A}^{\prime \prime}\right)$ holds, then

$$
\begin{aligned}
\frac{\left|K_{\alpha}(x, \bar{y})\right|^{2}}{K_{\alpha}(x, \bar{x}) K_{\alpha}(y, \bar{y})} & =\left[\frac{a(x, \bar{x}) a(y, \bar{y})}{|a(x, \bar{y})|^{2}}\right]^{\alpha} \cdot\left(1+O\left(\alpha^{-1}\right)\right) \\
& =e^{\alpha \psi(x \mid y)} \cdot\left(1+O\left(\alpha^{-1}\right)\right)
\end{aligned}
$$

as $\alpha \rightarrow+\infty$, and the result follows.

The significance of regularity is elucidated by the following fundamental lemma, taken from [Ber1].

Lemma 2.2. Let $z_{0}$ be a regular point of $\Omega, f$ a $C^{3}$ function on $\Omega$, and suppose that the integral

$$
\mathcal{I}(\alpha)=\alpha^{n} \int_{\Omega} f(x) e^{\alpha \psi\left(x \mid z_{0}\right)} d \mu(x)
$$

converges absolutely for $\alpha=\alpha_{1}$. Then it converges for all $\alpha>\alpha_{1}$, and as $\alpha \rightarrow+\infty$,

$$
\mathcal{I}(\alpha)=f\left(z_{0}\right)+\left[\Delta f\left(z_{0}\right)+\sigma\left(z_{0}\right) f\left(z_{0}\right)\right] \alpha^{-1}+o\left(\alpha^{-1}\right),
$$

where

$$
\sigma(z)=\frac{3}{2} \Delta \log g(z), \quad g(z)=\operatorname{det}\left(g_{j \bar{k}}(z)\right)_{j, k=1}^{n},
$$

and $\Delta$ is the Laplace-Beltrami operator on $\Omega$ (corresponding to the Riemannian metric $\left.\sum g_{j \bar{k}} d x_{j} d \bar{x}_{k}\right)$.

The action of $\Delta$ on functions on $\Omega$ is given by

$$
\Delta f(z)=\sum_{j, k=1}^{n} g^{\bar{j} k} \frac{\partial^{2} f}{\partial \bar{z}_{j} \partial z_{k}},
$$

where $\left(g^{\bar{j} k}\right)_{j, k=1}^{n}$ is the inverse of the matrix $\left(g_{j \bar{k}}\right)_{j, k=1}^{n}$; see e.g. [Ber1], Lemma 3 in the Appendix.

The proof of Lemma 2.2 can be found in [Ber1], Lemma 2.1. Before proceeding, let us put down some consequences of the assumptions we have made so far. 
Proposition 2.3. Suppose that $\left(\mathrm{A}^{\prime}\right)$ and $\left(\mathrm{E}^{\prime}\right)$ hold. Than for all $\alpha$ sufficiently large, the integral $\int_{\Omega} e^{\alpha \psi(x \mid y)} d \mu(x)$ exists for all $y \in \Omega$ and

$$
c_{n} \alpha^{n} \int_{\Omega} e^{\alpha \psi(x \mid y)} d \mu(x) \rightarrow 1
$$

as $\alpha \rightarrow+\infty$ through the set $E$.

If only $\left(\mathrm{A}^{\prime \prime}\right)$ and $\left(\mathrm{E}^{\prime}\right)$ hold, then for each $y \in \Omega$ the integral exists for all sufficiently large $\alpha \in E$, and (2.24) holds.

Proof. Let $0<\delta<1$. By $\left(\mathrm{A}^{\prime}\right)$, there exists $\alpha_{1}=\alpha_{1}(\delta)>0$ such that for all $\alpha \geq \alpha_{1}$ $(\alpha \in E)$ and $x, y \in \Omega$,

$$
\delta \leq\left|K_{\alpha}(x, \bar{y}) a(x, \bar{y})^{\alpha}\right| \cdot c_{n}^{-1} \alpha^{-n} \leq 1 / \delta
$$

Consequently,

$$
\delta^{3} \leq \frac{\left|K_{\alpha}(x, \bar{y})\right|^{2}}{K_{\alpha}(y, \bar{y})}\left[\frac{|a(x, \bar{y})|^{2}}{a(y, \bar{y})}\right]^{\alpha} \cdot c_{n}^{-1} \alpha^{-n} \leq \delta^{-3}
$$

and

$$
\delta^{3} \cdot c_{n} \alpha^{n} e^{\alpha \psi(x \mid y)} \leq \frac{\left|K_{\alpha}(x, \bar{y})\right|^{2}}{K_{\alpha}(y, \bar{y})} a(x, \bar{x})^{\alpha} \leq \delta^{-3} \cdot c_{n} \alpha^{n} e^{\alpha \psi(x \mid y)}
$$

Now the integral

$$
B_{\alpha} \mathbf{1}(y)=\int_{\Omega} \frac{\left|K_{\alpha}(x, \bar{y})\right|^{2}}{K_{\alpha}(y, \bar{y})} a(x, \bar{x})^{\alpha} d \mu(x)=\frac{\left\langle K_{\alpha}(\cdot, \bar{y}), K_{\alpha}(\cdot, \bar{y})\right\rangle}{K_{\alpha}(y, \bar{y})}=1
$$

exists and equals 1 whenever $K_{\alpha}(y, \bar{y}) \neq 0$ - in particular, for all $y \in \Omega$ if $\alpha \in E$. It follows that the integral in (2.24) exists for all $y$ when $\alpha \geq \alpha_{1}, \alpha \in E$; and since $\delta \in(0,1)$ is arbitrary, $(2.24)$ must hold. The proof for $\left(\mathrm{A}^{\prime \prime}\right)$ is similar.

Corollary 2.4. Suppose that $\left(\mathrm{A}^{\prime}\right)$ and $\left(\mathrm{E}^{\prime}\right)$ hold and let $f$ be a bounded measurable function on $\Omega$. Then for all sufficiently large $\alpha \in E$, the integral

$$
\int_{\Omega} f(x) e^{\alpha \psi(x \mid y)} d \mu(x)
$$

exists (i.e. converges absolutely) for all $y \in \Omega$.

If only $\left(\mathrm{A}^{\prime \prime}\right)$ and $\left(\mathrm{E}^{\prime}\right)$ hold, then for each $y \in \Omega$, the integral exists for all $\alpha \in E$ sufficiently large.

Proof. Since $0 \leq e^{\alpha \psi} \leq 1$ by Proposition 2.1, this is immediate from the preceding proposition. 
Theorem 2.5. Suppose that $\left(\mathrm{A}^{\prime \prime}\right)$ and $\left(\mathrm{E}^{\prime}\right)$ are fulfilled and $y \in \Omega$ is regular. Then for any function $f$ on $\Omega$ which is $C^{3}$ in a neighbourhood of $y$ and for which the integral defining the Berezin transform

$$
B_{\alpha} f(y)=\int_{\Omega} f(x) \frac{\left|K_{\alpha}(x, \bar{y})\right|^{2}}{K_{\alpha}(y, \bar{y})} a(x, \bar{x})^{\alpha} d \mu(x)
$$

exists in the absolute sense for some $\alpha=\alpha_{1}$ (for instance, this is the case when $f$ $i s$ bounded and measurable) this integral exists for all $\alpha \geq \alpha_{1}$ and satisfies

$$
B_{\alpha} f(y)=f(y)+\alpha^{-1} \Delta f(y)+o\left(\alpha^{-1}\right) \quad \text { as } \alpha \rightarrow+\infty .
$$

Proof. By $\left(\mathrm{A}^{\prime \prime}\right)$,

$$
\left|K_{\alpha}(x, \bar{y})\right|=|a(x, \bar{y})|^{-\alpha} \cdot c_{n} \alpha^{n}\left[1+\frac{B(x, \bar{y})}{c_{n} \alpha}+\frac{C(x, \bar{y}, \alpha)}{c_{n} \alpha^{2}}\right],
$$

hence

$$
\frac{\left|K_{\alpha}(x, \bar{y})\right|^{2}}{K_{\alpha}(y, \bar{y})}=\left[\frac{a(y, \bar{y})}{|a(x, \bar{y})|^{2}}\right]^{\alpha} \cdot c_{n} \alpha^{n}\left[1+\frac{\widetilde{B}(x, y)}{\alpha}+\frac{\widetilde{C}(x, y, \alpha)}{\alpha^{2}}\right],
$$

where $\widetilde{B}(x, y)=c_{n}^{-1}[B(x, \bar{y})+B(y, \bar{x})-B(y, \bar{y})]$ and $\widetilde{C}(x, y, \alpha)$ are bounded functions on $\Omega \times\{y\}$ and $\Omega \times\{y\} \times E$, respectively. Substituting (2.29) into (2.28), we obtain

$$
\begin{aligned}
B_{\alpha} f(y)=c_{n} & \alpha^{n} \int_{\Omega} f(x) e^{\alpha \psi(x \mid y)} d \mu(x) \\
& +c_{n} \alpha^{n-1} \int_{\Omega} f(x) \widetilde{B}(x, y) e^{\alpha \psi(x \mid y)} d \mu(x) \\
& +c_{n} \alpha^{n-2} \int_{\Omega} f(x) \widetilde{C}(x, y, \alpha) e^{\alpha \psi(x \mid y)} d \mu(x) .
\end{aligned}
$$

The absolute value of the last summand does not exceed

$$
\sup |\widetilde{C}(x, y, \alpha)| \cdot c_{n} \alpha^{n-2} \int_{\Omega}|f(x)| e^{\alpha \psi(x \mid y)} d \mu(x) .
$$

Owing to the hypothesis on $f$ and since $\widetilde{B}(\cdot, y)$ is bounded and $C^{3}$ on $\Omega$, Lemma 2.2 can be applied to the first two integrals in (2.30) and to the integral in (2.31).

This gives

$$
\begin{aligned}
c_{n}^{-1} B_{\alpha} f(y) & =f(y)+\alpha^{-1}[\Delta f(y)+\sigma(y) f(y)]+\alpha^{-1}[f(y) \widetilde{B}(y, y)]+o\left(\alpha^{-1}\right) \\
& =f(y)+\alpha^{-1}\left[\Delta f(y)+\sigma(y) f(y)+c_{n}^{-1} B(y, \bar{y}) f(y)\right]+o\left(\alpha^{-1}\right)
\end{aligned}
$$

as $\alpha \rightarrow+\infty$. Taking $f \equiv \mathbf{1}$ we have, in particular,

$$
c_{n}^{-1}=1+\alpha^{-1}\left[\sigma(y)+c_{n}^{-1} B(y, \bar{y})\right]+o\left(\alpha^{-1}\right) \quad \text { as } \alpha \rightarrow+\infty .
$$

Thus necessarily

$$
c_{n}=1
$$

and

$$
B(y, \bar{y})=-\sigma(y)
$$

Therefore

$$
B_{\alpha} f(y)=f(y)+\alpha^{-1} \Delta f(y)+o\left(\alpha^{-1}\right)
$$

as $\alpha \rightarrow+\infty$, as asserted.

En route, we have proved the following interesting corollary. 
Corollary 2.6. Suppose that $\left(\mathrm{A}^{\prime \prime}\right)$ and $\left(\mathrm{E}^{\prime}\right)$ are fulfilled and that there exists at least one regular point on $\Omega$. Then $c_{n}=1$ and

$$
B(x, \bar{x})=-\frac{3}{2} \Delta \log g(x)
$$

at each regular point $x \in \Omega$.

We are now ready to state our next (and last) assumption.

Assumption $\left(\mathrm{C}^{\prime}\right)$. All points of $\Omega$ are regular.

This is quite different from Berezin's (C). However, an examination of [Ber1] reveals that the only time $(\mathrm{C})$ was used was when establishing that all points are regular. In practice, it is usually easier and more straightforward to verify $\left(\mathrm{C}^{\prime}\right)$ than $(\mathrm{C})$. Besides, $\left(\mathrm{C}^{\prime}\right)$ somehow seems to be more natural.

A point $y \in \Omega$ will be called distinguished if

$$
a(\cdot, \bar{y}) \equiv 1
$$

or, equivalently, $\Psi(\cdot, \bar{y}) \equiv 0$. Berezin's assumption (D) was that there exists a distinguished point on $\Omega$. We will see that we can do without this assumption; but, for the sake of completeness, we will briefly discuss it below and indicate some of its consequences.

As was noted in the Introduction, (D) is just a normalization condition. To see this, observe that the Kähler potential $\Psi$ is not determined uniquely: if $\Psi(x)$ is a real-valued solution of (2.9), then so is

$$
\Psi^{1}(x)=\Psi(x)+\log |G(x)|^{2}
$$

for any zero-free analytic function $G(x)$ on $\Omega$. The effects of passing from $\Psi$ to $\Psi^{1}$ are summarized by the next proposition.

\section{Proposition 2.7.}

(i) If $a(x, \bar{y})$ satisfies $\left(\mathrm{E}^{\prime}\right)$ for $\Psi$ (with $\left.\alpha_{0}=1\right)$, then $a^{1}(x, \bar{y})=G(x) \overline{G(y)} a(x, \bar{y})$ satisfies $\left(\mathrm{E}^{\prime}\right)$ for $\Psi^{1}$ (with $\left.\alpha_{0}=1\right)$.

(ii) The operator $U: f \mapsto f_{1}, f_{1}(x)=G(x)^{-\alpha} f(x)$, is a Hilbert space isomorphism of $A_{\alpha}^{2}(\Psi)$ onto $A_{\alpha}^{2}\left(\Psi^{1}\right)$, for any positive integer $\alpha$.

(iii) The reproducing kernels for $\Psi$ and $\Psi^{1}$ are related by

$$
K^{1}(x, \bar{y})=G(x)^{-\alpha} K(x, \bar{y}) \overline{G(y)^{-\alpha}}
$$

(iv) $\left(\mathrm{A}^{\prime}\right)$ is fulfilled for $\Psi$ iff it is fulfilled for $\Psi^{1}$. Similarly for $\left(\mathrm{A}^{\prime \prime}\right)$.

(v) $\left(\mathrm{C}^{\prime}\right)$ is fulfilled for $\Psi$ iff it is fulfilled for $\Psi^{1}$; in fact, $\psi=\psi^{1}$.

(vi) The Berezin transforms for $\Psi$ and $\Psi^{1}$ coincide: $B_{\alpha}=B_{\alpha}^{1}$.

(vii) If $A$ is a bounded linear operator on $A_{\alpha}^{2}(\Psi)$, then the covariant symbol of $A$ (with respect to $\Psi$ ) coincides with the covariant symbol of $U A U^{*}$ (with respect to $\left.\Psi^{1}\right)$. Consequently, the algebra of covariant symbols with respect to $\Psi$ coincides with the one for $\Psi^{1}$. 
Proof. Straightforward verification.

Proposition 2.7 is very important, since it makes the whole theory meaningful: it shows that the Berezin transform, the quantization algebras, and other related objects depend only on the Kähler structure $\omega$ on $\Omega$, and not on the potential by means of which they were defined. (From the viewpoint of physics, passage from $\Phi$ to $\Phi^{1}$ amounts to a "gauge transformation"). If $\Psi$ is a choice for the Kähler potential, then taking

$$
G(x)=\sqrt{a\left(y_{0}, \bar{y}_{0}\right)} / a\left(x, \bar{y}_{0}\right)
$$

gives a potential which has $y_{0}$ as a distinguished point.

Proposition 2.8. Assume that $\left(\mathrm{A}^{\prime \prime}\right),\left(\mathrm{E}^{\prime}\right)$ and $(\mathrm{D})$ are fulfilled. Then the constant function $\mathbf{1}$ belongs to $A_{\alpha}^{2}$ for $\alpha \in E$ sufficiently large, and $\|\mathbf{1}\|_{\alpha}^{2} \sim c_{n}^{-1} \alpha^{-n}$ as $\alpha \rightarrow+\infty$.

Proof. Let $y_{0}$ be the distinguished point. By Proposition 2.3,

$$
\int_{\Omega} e^{\alpha\left(x \mid y_{0}\right)} d \mu(x) \sim c_{n}^{-1} \alpha^{-n} \quad \text { as } \alpha \rightarrow+\infty,
$$

and the integral exists for all $\alpha$ sufficiently large. On the other hand, as $e^{\psi\left(x \mid y_{0}\right)}=$ $e^{-\Psi(x)}=a(x)$, we have

$$
\|\mathbf{1}\|_{\alpha}^{2}=\int_{\Omega} a(x)^{\alpha} d \mu(x)=\int_{\Omega} e^{\alpha \psi\left(x \mid y_{0}\right)} d \mu(x)
$$

and the assertion follows.

Corollary 2.9. Assume that $\left(\mathrm{A}^{\prime \prime}\right),\left(\mathrm{E}^{\prime}\right)$ and $(\mathrm{D})$ hold. Then there exists an $\alpha_{\Omega}>0$ such that $H^{\infty}(\Omega) \subset A_{\alpha}^{2}$ for $\alpha \geq \alpha_{\Omega}, \alpha \in E$.

The careful reader has certainly noticed that we have no counterpart for Berezin's assumption (B). Here is an explanation. In [Ber1], the condition (B) was used twice: first, for establishing that all points of $\Omega$ are regular - which we have taken care of by our requirement $\left(\mathrm{C}^{\prime}\right)$ - and, second, for showing that the condition (2c) from (our) definition of quantization was fulfilled, i.e. that there were enough "quantizable" functions to separate points of $\Omega$. However, Berezin's proof of the latter assertion seems to contain a gap, which the present author is unable to fix. ${ }^{5}$ This would leave Theorem 2.3 of [Ber1] unproved, and the whole theory would break down.

\footnotetext{
${ }^{5}$ Towards the end of the proof of Theorem 2.3 in [Ber1], Berezin applies Lemma 2.1 (= our Lemma 2.2) to the integral (in our notation)

$$
\alpha^{n} \int_{\Omega} \chi(z) e^{\alpha \psi\left(z \mid z_{0}\right)} d \mu(z)
$$

where $\chi(z)=|g(z)|^{2} \cdot|a(z, \bar{v})|^{2 \beta}, g \in A_{\beta}^{2}, \beta>0$, and $z_{0}$ is a distinguished point. However, the application is legitimate only if the integral is known to exist for some $\alpha$ ! It's far from clear why this should be so, and, in fact, we will exhibit a situation (cf. Remark 3.22) when the integral diverges for arbitrarily large $\alpha$.
} 
Fortunately (?), there seems to be one more mistake in [Ber1], this time in the definition of the set $\mathfrak{B}$ right before Theorem 2.2 of [Ber1]. ${ }^{6}$ Miraculously, by modifying slightly Berezin's argument, these two mistakes can be made to "cancel out" each other (so the validity of the results in [Ber1] remains unaffected). Moreover, it turns out that Berezin's assumption (B) is no longer necessary, since all the things it was needed for can be established with the aid of just $\left(\mathrm{A}^{\prime}\right),\left(\mathrm{C}^{\prime}\right)$ and $\left(\mathrm{E}^{\prime}\right)$. (Compare the proof of Theorem 2.3 in [Ber1] with that of our Theorem 2.11 below.)

Now consider a number $h>0$ such that $\alpha=1 / h \in E ; h$ has the interpretation of "Planck's constant". Following the construction described at the beginning of this section, we let $\mathcal{A}_{h}$ be the algebra of covariant symbols for the Hilbert space $A_{\alpha}^{2}$. Let $\mathcal{A}$ be the set of all functions $f(h \mid x), h \in E^{*}=\{1 / \alpha, \alpha \in E\}, x \in \Omega$, for which $f(h \mid \cdot) \in \mathcal{A}_{h}$ for all $h$ and the limit

$$
\lim _{h \rightarrow 0+} f(h \mid x) \stackrel{\text { def }}{=} f(0 \mid x)
$$

exists for all $x \in \Omega$. Equipped with the "fiberwise" operations

$$
\begin{gathered}
(f+g)(h \mid x)=f(h \mid x)+g(h \mid x), \\
(c f)(h \mid x)=c f(h \mid x), \\
(f * g)(h \mid x)=\left(f(h \mid \cdot) *_{h} g(h \mid \cdot)\right)(x)
\end{gathered}
$$

$\left(*_{h}\right.$ is the product in $\left.\mathcal{A}_{h}\right), \mathcal{A}$ becomes an associative algebra. It's clear that $\mathcal{A}$ satisfies the conditions (1), (1a), (1b), (1c) from our definition of quantization. Denote further by $\widetilde{\mathcal{A}}$ the linear subset of $\mathcal{A}$ consisting of all functions $f(h \mid x)$ for which the following two conditions are fulfilled:

$(\widetilde{\mathcal{A}} 1) f$ can be written in the form

$$
f(h \mid x)=f(0 \mid x, \bar{x})+h f_{1}(x, \bar{x})+h^{2} f_{2}(h \mid x, \bar{x}),
$$

where $f(0 \mid x, \bar{y}), f_{1}(x, \bar{y})$, and $f_{2}(h \mid x, \bar{y})$ (the last, for each fixed $h$ ) are (singlevalued) sesquianalytic functions on $\Omega \times \Omega$;

$(\widetilde{\mathcal{A}} 2)$ there exists $h_{0}>0$ such that these sesquianalytic functions satisfy

$$
h^{-n} \int_{\Omega}\left[|f(0 \mid x, \bar{y})|^{2}+\left|f_{1}(x, \bar{y})\right|^{2}+\left|f_{2}(h \mid x, \bar{y})\right|^{2}\right] e^{\frac{1}{h} \psi(x \mid y)} d \mu(x) \leq r(y)
$$

and

$$
h^{-n} \int_{\Omega}\left[|f(0 \mid y, \bar{x})|^{2}+\left|f_{1}(y, \bar{x})\right|^{2}+\left|f_{2}(h \mid y, \bar{x})\right|^{2}\right] e^{\frac{1}{h} \psi(x \mid y)} d \mu(x) \leq r(y)
$$

for all $h \in E^{*} \cap\left(0, h_{0}\right)$, with $r(y)<+\infty$ independent of $h$.

(Note that our condition $(\widetilde{\mathcal{A}} 2)$ is different from that of Berezin [Ber1]; see footnote ${ }^{6}$ above.) We are going to show that, granted the assumptions $\left(\mathrm{A}^{\prime}\right),\left(\mathrm{C}^{\prime}\right)$ and $\left(\mathrm{E}^{\prime}\right)$, $\mathcal{A}$ is a quantization satisfying the weak form of the correspondence principle.

\footnotetext{
${ }^{6}$ In the last displayed formula before the statement of Theorem 2.2 in [Ber1], the $e^{-\frac{1}{h} \Phi(z, \bar{z})}$ in the integrand should be changed to $e^{\frac{1}{h} \phi(z, \bar{z} \mid v, \bar{v})}$.
} 
Theorem 2.10. Assume that $\left(\mathrm{A}^{\prime \prime}\right),\left(\mathrm{E}^{\prime}\right)$ and $\left(\mathrm{C}^{\prime}\right)$ are fulfilled and $f, g \in \widetilde{\mathcal{A}}$. Then as $E^{*} \ni h \rightarrow 0$,

$$
\begin{gathered}
(f * g)(h \mid z)=f(0 \mid z) g(0 \mid z)+o(1), \\
\frac{1}{h}(f * g-g * f)(h \mid z)=\frac{1}{i}\{f(0 \mid \cdot), g(0 \mid \cdot)\}(z)+o(1),
\end{gathered}
$$

where $\{\cdot, \cdot\}$ is the Poisson bracket on $\Omega$.

Proof. Fix $z \in \Omega$. First note that ( $\widetilde{\mathcal{A}} 1)$ furnishes an explicit sesquianalytic extension $f(h \mid x, \bar{y})$ of $f(h \mid x)$ for each $h \in E^{*}$; the operator of which $f(h \mid \cdot)$ is the covariant symbol is then the integral operator on $\Omega$ with the kernel $f(h \mid x, \bar{y}) K_{\alpha}(x, \bar{y}), \alpha=1 / h$ — see (2.4). Similarly for $g$. Hence by (2.14),

$$
(f * g)(h \mid z)=\int_{\Omega} f(h \mid z, \bar{x}) g(h \mid x, \bar{z}) \frac{\left|K_{\alpha}(x, \bar{z})\right|^{2}}{K_{\alpha}(z, \bar{z})} a(x, \bar{x})^{\alpha} d \mu(x), \quad \alpha \equiv 1 / h .
$$

Denote $u(h \mid x, \bar{y})=f(h \mid z, \bar{y}) g(h \mid x, \bar{z}), x, y \in \Omega, h \in E^{*}$. In view of $(\widetilde{\mathcal{A}} 1), u$ can be written in the form

$$
u(h \mid x, \bar{y})=u(0 \mid x, \bar{y})+h u_{1}(x, \bar{y})+h^{2} u_{2}(h \mid x, \bar{y}),
$$

with

$$
\begin{gathered}
u(0 \mid x, \bar{y})=f(0 \mid z, \bar{y}) g(0 \mid x, \bar{z}), \\
u_{1}(x, \bar{y})=f(0 \mid z, \bar{y}) g_{1}(x, \bar{z})+g(0 \mid x, \bar{z}) f_{1}(z, \bar{y}), \\
u_{2}(h \mid x, \bar{y})=f(0 \mid z, \bar{y}) g_{2}(h \mid x, \bar{z})+f_{2}(h \mid z, \bar{y}) g(0 \mid x, \bar{z})+f_{1}(z, \bar{y}) g_{1}(x, \bar{z}) .
\end{gathered}
$$

The formula (2.36) can then be written in the form

$$
\begin{aligned}
(f * g)(h \mid z) & =\int_{\Omega} u(h \mid x, \bar{x}) \frac{\left|K_{\alpha}(x, \bar{z})\right|^{2}}{K_{\alpha}(z, \bar{z})} a(x, \bar{x})^{\alpha} d \mu(x) \\
& =\int u(0 \mid x, \bar{x}) \ldots+h \int u_{1}(x, \bar{x}) \ldots+h^{2} \int u_{2}(h \mid x, \bar{x}) \ldots
\end{aligned}
$$

Owing to $(\widetilde{\mathcal{A}} 2)$, the function $u_{2}$ satisfies, for all $h \in\left(0, h_{0}\right) \cap E^{*}$,

$$
h^{-n} \int_{\Omega}\left|u_{2}(h \mid x, \bar{x})\right| e^{\alpha \psi(x \mid z)} d \mu(x) \leq r(z)<+\infty,
$$

with $r(z)$ independent of $h$. In view of the formula (2.25) in the proof of Proposition 2.3 , this is equivalent to

$$
\int_{\Omega}\left|u_{2}(h \mid x, \bar{x})\right| \frac{\left|K_{\alpha}(x, \bar{z})\right|^{2}}{K_{\alpha}(z, \bar{z})} a(x, \bar{x})^{\alpha} d \mu(x) \leq r(z)<+\infty
$$

for all $h=1 / \alpha \in\left(0, h_{0}\right) \cap E^{*}$, with $r(z)$ independent of $h$. It follows that the last summand in $(2.37)$ is of order $O\left(h^{2}\right)$. One more exploitation of $(\widetilde{\mathcal{A}} 2)$ shows that an application of Theorem 2.5 to the first two summands is legitimate. Therefore

$$
(f * g)(h \mid z)=u(0 \mid z, \bar{z})+\left.h \cdot \Delta u(0 \mid x, \bar{x})\right|_{x=z}+h \cdot u_{1}(z, \bar{z})+o(h) .
$$


In view of (2.23),

$$
\Delta u(0 \mid x, \bar{x})=\Delta_{x \bar{x}}[f(0 \mid z, \bar{x}) g(0 \mid x, \bar{z})]=\sum_{j, k} g^{\bar{j} k} \frac{\partial f(0 \mid z, \bar{x})}{\partial \bar{x}_{j}} \frac{\partial g(0 \mid x, \bar{z})}{\partial x_{k}},
$$

so we finally arrive at

$$
\begin{aligned}
&(f * g)(h \mid z)=f(0 \mid z, \bar{z}) g(0 \mid z, \bar{z})+h \cdot\left[f(0 \mid z, \bar{z}) g_{1}(z, \bar{z})+g(0 \mid z, \bar{z}) f_{1}(z, \bar{z})\right] \\
&+h \cdot\left[\sum_{j, k} g^{j} k \frac{\partial f(0 \mid z, \bar{z})}{\partial \bar{z}_{j}} \frac{\partial g(0 \mid z, \bar{z})}{\partial z_{k}}\right]+o(h),
\end{aligned}
$$

and (2.34) immediately follows. Interchanging $f$ and $g$ and subtracting, we obtain $\frac{1}{h}(f * g-g * f)(h \mid z)=\sum_{j, k} g^{\bar{j} k}\left(\frac{\partial f(0 \mid z, \bar{z})}{\partial \bar{z}_{j}} \frac{\partial g(0 \mid z, \bar{z})}{\partial z_{k}}-\frac{\partial g(0 \mid z, \bar{z})}{\partial \bar{z}_{j}} \frac{\partial f(0 \mid z, \bar{z})}{\partial z_{k}}\right)+o(1)$.

Since the Poisson bracket is defined by

$$
\{F, G\}=i \sum_{j, k} g^{\bar{j} k}\left(\frac{\partial F}{\partial \bar{z}_{j}} \frac{\partial G}{\partial z_{k}}-\frac{\partial G}{\partial \bar{z}_{j}} \frac{\partial F}{\partial z_{k}}\right)
$$

the assertion (2.35) follows, and the proof is complete.

Theorem 2.11. Assume that $\left(\mathrm{A}^{\prime}\right),\left(\mathrm{C}^{\prime}\right)$ and $\left(\mathrm{E}^{\prime}\right)$ are fulfilled. Then for any two distinct points $z_{0}, z_{1} \in \Omega$ there exists a function $f \in \widetilde{\mathcal{A}}$ such that $f\left(0 \mid z_{0}\right) \neq f\left(0 \mid z_{1}\right)$.

Proof. Consider the function

$$
g(z)=\left[\sqrt{a\left(z_{0}, \bar{z}_{0}\right)} / a\left(z, \bar{z}_{0}\right)\right]^{\alpha_{1}}, \quad \alpha_{1}>0 .
$$

We have

$$
\begin{aligned}
\|g\|_{\alpha_{1}}^{2} & =\int_{\Omega} a\left(z_{0}, \bar{z}_{0}\right)^{\alpha_{1}} a\left(z, \bar{z}_{0}\right)^{-\alpha_{1}} a\left(z_{0}, \bar{z}\right)^{-\alpha_{1}} a(z, \bar{z})^{\alpha_{1}} d \mu(z) \\
& =\int_{\Omega} e^{\alpha_{1} \psi\left(z \mid z_{0}\right)} d \mu(z)
\end{aligned}
$$

which is finite for sufficiently large $\alpha_{1}$, by Proposition 2.3. Fix such an $\alpha_{1}$ from now on. Choose a $C^{\infty}$ function $\epsilon(h)$ on $(0,+\infty)$ such that $\epsilon(h)=1$ on some interval $0<h<h_{0}$, and $\epsilon(h)=0$ for $h \geq 1 / \alpha_{1}$. Let

$$
\begin{aligned}
f(h \mid x) & =\epsilon(h) \cdot g(x) \overline{g(x)} a(x, \bar{x})^{\alpha_{1}} \\
& =\epsilon(h) \cdot e^{\alpha_{1} \psi\left(x \mid z_{0}\right)} .
\end{aligned}
$$

We claim that $f$ has the required properties. First of all, it's clear from the regularity of $z_{0}$ that $f(0 \mid x)=e^{\alpha_{1} \psi\left(x \mid z_{0}\right)}$ separates $z_{0}$ from all other points of $\Omega: f\left(0 \mid z_{0}\right)=1$, while $f(0 \mid z)<1$ for all $z \neq z_{0}$. 
Since $\epsilon(h) \equiv 1$ on $\left(0, h_{0}\right),(\widetilde{\mathcal{A}} 1)$ is trivially satisfied, with $f_{1}=f_{2}=0$ and

$$
f(0 \mid x, \bar{y})=g(x) \overline{g(y)} a(x, \bar{y})^{\alpha_{1}} .
$$

To check $(\widetilde{\mathcal{A}} 2)$, observe that

$$
0 \leq e^{\left(\alpha-\alpha_{1}\right) \psi(x \mid y)} \leq 1 \quad \text { for all } \alpha>\alpha_{1} \text { and } x, y \in \Omega .
$$

Hence

$$
e^{\alpha \psi(x \mid y)} \leq e^{\alpha_{1} \psi(x \mid y)}=a(x, \bar{x})^{\alpha_{1}} a(y, \bar{y})^{\alpha_{1}} \cdot\left|a(x, \bar{y})^{-\alpha_{1}}\right|^{2},
$$

or

$$
\left|a(x, \bar{y})^{\alpha_{1}}\right|^{2} e^{\alpha \psi(x \mid y)} \leq a(x, \bar{x})^{\alpha_{1}} a(y, \bar{y})^{\alpha_{1}} .
$$

It follows that

$$
\begin{aligned}
\int_{\Omega}\left|g(x) a(x, \bar{y})^{\alpha_{1}}\right|^{2} e^{\alpha \psi(x \mid y)} d \mu(x) & \leq a(y, \bar{y})^{\alpha_{1}} \int_{\Omega}|g(x)|^{2} a(x, \bar{x})^{\alpha_{1}} d \mu(x) \\
& =a(y, \bar{y})^{\alpha_{1}} \cdot\|g\|_{\alpha_{1}}^{2}<+\infty
\end{aligned}
$$

for all $\alpha>\alpha_{1}$. Hence, an application of Lemma 2.2 to the integral

$$
\int_{\Omega} \alpha^{n}|f(0 \mid x, \bar{y})|^{2} e^{\alpha \psi(x \mid y)} d \mu(x)=\alpha^{n}|g(y)|^{2} \int_{\Omega}\left|g(x) a(x, \bar{y})^{\alpha_{1}}\right|^{2} e^{\alpha \psi(x \mid y)} d \mu(x)
$$

is legitimate, and shows that this integral has a finite limit $|g(y)|^{4} a(y, \bar{y})^{2 \alpha_{1}}$ as $\alpha \rightarrow+\infty$. It must therefore be uniformly bounded for all large $\alpha$, which settles $(\widetilde{\mathcal{A}} 2)$.

It remains to show that $f(h \mid \cdot)$ is in $\mathcal{A}_{h}$ for each $h$, i.e. is the covariant symbol of a bounded linear operator on $A_{\alpha}^{2}$ (as usual, $\alpha=1 / h$ ). By (2.4), this linear operator $A_{h}$ is the integral operator on $\Omega$ with the kernel $f(h \mid x, \bar{y}) K_{\alpha}(x, \bar{y})$ :

$$
A_{h} F(x)=\int_{\Omega} f(h \mid x, \bar{y}) K_{\alpha}(x, \bar{y}) F(y) a(y, \bar{y})^{\alpha} d \mu(y), \quad \text { for } F \in A_{\alpha}^{2}
$$

That is,

$$
A_{h} F(x)=\epsilon(h) g(x) \int_{\Omega} \overline{g(y)} a(x, \bar{y})^{\alpha_{1}} K_{\alpha}(x, \bar{y}) F(y) a(y, \bar{y})^{\alpha} d \mu(y) .
$$

Obviously, it suffices to treat the case when $\epsilon(h) \neq 0$, i.e. $\alpha>\alpha_{1}$. Denote the integral on the right-hand side by $\mathcal{I}(x)$. By the Schwarz inequality,

$$
|\mathcal{I}(x)|^{2} \leq\|F\|_{\alpha}^{2} \int_{\Omega}|g(y)|^{2} \cdot\left|K_{\alpha}(x, \bar{y}) a(x, \bar{y})^{\alpha_{1}}\right|^{2} \cdot a(y, \bar{y})^{\alpha} d \mu(y) .
$$

Owing to $\left(\mathrm{A}^{\prime}\right)$, there exists $\alpha_{\Omega}>0$ such that, for all $\alpha>\alpha_{\Omega}, \alpha \in E$, and $x, y \in \Omega$,

$$
\frac{1}{2} \leq\left|\frac{K_{\alpha}(x, \bar{y})}{\alpha^{n} a(x, \bar{y})^{-\alpha}}\right| \leq 2
$$


Without loss of generality, we may assume that $\alpha_{1}$ was chosen to be bigger than $\alpha_{\Omega}$. Then for all $\alpha>\alpha_{1}$,

$$
\begin{aligned}
\left|K_{\alpha}(x, \bar{y}) a(x, \bar{y})^{\alpha_{1}}\right|^{2} & \leq 4 \alpha^{2 n}|a(x, \bar{y})|^{-2\left(\alpha-\alpha_{1}\right)} \\
& \leq 4 \alpha^{2 n} a(x, \bar{x})^{-\left(\alpha-\alpha_{1}\right)} a(y, \bar{y})^{-\left(\alpha-\alpha_{1}\right)},
\end{aligned}
$$

since, according to Proposition 2.1,

$$
a(x, \bar{x}) a(y, \bar{y})|a(x, \bar{y})|^{-2}=e^{\psi(x \mid y)} \leq 1 .
$$

Consequently, the integral in (2.39) is dominated by

$$
4 \alpha^{2 n} a(x, \bar{x})^{\alpha_{1}-\alpha} \int_{\Omega}|g(y)|^{2} a(y, \bar{y})^{\alpha_{1}} d \mu(y)=4 \alpha^{2 n} a(x, \bar{x})^{\alpha_{1}-\alpha}\|g\|_{\alpha_{1}}^{2},
$$

and

$$
\left|A_{h} F(x)\right|^{2} \leq \epsilon(h)^{2}|g(x)|^{2}\|F\|_{\alpha}^{2} \cdot 4 \alpha^{2 n} a(x, \bar{x})^{\alpha_{1}-\alpha}\|g\|_{\alpha_{1}}^{2}
$$

Therefore

$$
\begin{aligned}
\left\|A_{h} F\right\|^{2} & \leq 4 \alpha^{2 n}\|g\|_{\alpha_{1}}^{2} \epsilon(h)^{2}\|F\|_{\alpha}^{2} \int_{\Omega}|g(x)|^{2} a(x, \bar{x})^{\alpha_{1}-\alpha} \cdot a(x, \bar{x})^{\alpha} d \mu(x) \\
& =4 \alpha^{2 n}\|g\|_{\alpha_{1}}^{2} \epsilon(h)^{2}\|F\|_{\alpha}^{2} \cdot\|g\|_{\alpha_{1}}^{2} .
\end{aligned}
$$

Thus

$$
\left\|A_{h}\right\| \leq 2 \alpha^{n} \epsilon(h)\|g\|_{\alpha_{1}}^{2}<+\infty \quad \text { for all } \alpha>\alpha_{1}, \alpha \in E, h=1 / \alpha,
$$

i.e. $A_{h}$ is a bounded linear operator on $A_{1 / h}^{2}$ for all $h>0, h \in E^{*}$. The proof is finished.

The proofs of the last two theorems were modelled on the proofs of Theorems 2.2 and 2.3 in [Ber1].

Observe that $(2.40)$ is the only place where $\left(\mathrm{A}^{\prime}\right)$ was used in full; in the rest $(=$ Theorem 2.10 and before), what was needed was only its "non-uniform" analogue $\left(\mathrm{A}^{\prime \prime}\right)$. It would certainly be pleasing to have a proof of Theorem 2.11 which would use only $\left(\mathrm{A}^{\prime \prime}\right)$ instead of $\left(\mathrm{A}^{\prime}\right)$. In the particular case when $\Omega$ is a bounded domain in $\mathbf{C}^{n}$, this can be done.

Theorem 2.11A. Assume that $\left(\mathrm{A}^{\prime \prime}\right)$ and $\left(\mathrm{E}^{\prime}\right)$ are fulfilled and that $\Omega$ is a bounded domain in $\mathbf{C}^{n}$. Then for any two distinct points $z_{0}, z_{1} \in \Omega$ there exists a function $f \in \widetilde{\mathcal{A}}$ such that $f\left(0 \mid z_{0}\right) \neq f\left(0 \mid z_{1}\right)$.

Proof. Let $g \in H^{\infty}(\Omega)$ be a bounded analytic function on $\Omega$. The multiplication operator

$$
M_{g}: f \longmapsto g f, \quad f \in A_{\alpha}^{2},
$$

is then a bounded $\left(\left\|M_{g}\right\| \leq\|g\|_{\infty}\right)$ linear operator on $A_{\alpha}^{2}$, for any $\alpha>0$. Let us find the covariant symbol of $M_{g}$ as an operator on $A_{\alpha}^{2}$. Since

$$
\widetilde{M}_{g}(x, \bar{y})=\frac{\left\langle g(\cdot) K_{\alpha}(\cdot, \bar{y}), K_{\alpha}(\cdot, \bar{x})\right\rangle}{K_{\alpha}(x, \bar{y})}=\frac{g(x) K_{\alpha}(x, \bar{y})}{K_{\alpha}(x, \bar{y})}=g(x),
$$


we have $\widetilde{M}_{g}(x)=g(x)$, for all $\alpha>0$. Thus, the function

$$
f(h \mid x, \bar{y}) \stackrel{\text { def }}{=} g(x), \quad x, y \in \Omega, h \in E^{*},
$$

belongs to $\mathcal{A}$, and $f(0 \mid x)=g(x)$.

We claim that $f \in \widetilde{\mathcal{A}}$. Indeed, $(\widetilde{\mathcal{A}} 1)$ is obviously fulfilled, with $f_{1}=f_{2}=0$, and $(\widetilde{\mathcal{A}} 2)$ reduces to showing that there is an $\alpha_{1}>0$ such that

$$
\alpha^{n} \int_{\Omega}|g(x)|^{2} e^{\alpha \psi(x \mid y)} d \mu(x) \leq r(y)<+\infty \quad \forall \alpha>\alpha_{1},
$$

where $r(y)$ is independent of $\alpha$. However, as $g$ is bounded, this follows immediately from Corollary 2.4. Hence, $f \in \widetilde{\mathcal{A}}$.

Finally, let $z_{0}, z_{1}$ be two distinct points and assume that $\Omega$ is bounded. Then the coordinate functions belong to $H^{\infty}(\Omega)$, so there exists $g \in H^{\infty}(\Omega)$ such that $g\left(z_{0}\right) \neq g\left(z_{1}\right)$. The above construction then gives $f \in \widetilde{\mathcal{A}}$ such that $f\left(0 \mid z_{0}\right) \neq$ $f\left(0 \mid z_{1}\right)$, and the proof is finished.

Summarizing our results so far, we have proved

Theorem 2.12. If $\left(\mathrm{A}^{\prime}\right),\left(\mathrm{C}^{\prime}\right)$ and $\left(\mathrm{E}^{\prime}\right)$ hold, the algebra $\mathcal{A}$ is a quantization of $(\Omega, \omega)$ satisfying the weak form of the correspondence principle.

Proof. The validity of the conditions (1), (1a), (1b), and (1c) in the definition of quantization is apparent from the way in which $\mathcal{A}$ was constructed; $(2 \mathrm{a})$ and $(2 \mathrm{~b})$ are the contents of Theorem 2.10, and (2c) is Theorem 2.11 .

Theorem 2.12A. Assume that $\Omega$ is a bounded domain in $\mathbf{C}^{n}$ and $\left(\mathrm{A}^{\prime \prime}\right),\left(\mathrm{C}^{\prime}\right)$ and $\left(\mathrm{E}^{\prime}\right)$ are fulfilled. Then the algebra $\mathcal{A}$ is a quantization of $(\Omega, \omega)$ satisfying the weak form of the correspondence principle.

We conclude this section by four examples. The first two $\left(\Omega=\mathbf{C}^{n}\right.$ and $\Omega$ a bounded symmetric domain) are the ones treated by Berezin in [Ber1]. The third one (the cylinder; or, punctured plane) shows how to pass to the universal cover of $\Omega$ in case when the Kähler potential $\Psi$ is not globally defined. The fourth $(\Omega$ a planar domain with the Poincaré metric), treated in detail in [E3], uses a similar machinery; moreover, both of them also show that it's sometimes possible to make things work even when the crucial assumption, $\left(\mathrm{A}^{\prime}\right)$, is not fulfilled. Examples illustrating a direct application of the method developed in this section will be given in Section 3.

Example 2.13. $\Omega=\mathbf{C}^{n}$, with the usual (euclidean) Kähler form $\omega=\sum_{k} d z_{k} \wedge d \bar{z}_{k}$. Thus $g_{j \bar{k}}=\delta_{j k}$ for all $j, k$, and we can take $\Psi(z)=\sum_{k} z_{k} \bar{z}_{k} \equiv\|z\|^{2}$ as a potential. The condition $\left(\mathrm{E}^{\prime}\right)$ is then satisfied (with $\alpha_{0}=1$ ) with

$$
a(x, \bar{y})=e^{-x \cdot \bar{y}}, \quad x \cdot \bar{y}=\sum_{k} x_{k} \bar{y}_{k} .
$$

$A_{\alpha}^{2}$ is the Segal-Bargmann (or Fock) space of all entire functions on $\mathbf{C}^{n}$ which are square-integrable with respect to the Gaussian measure $e^{-\alpha\|x\|^{2}} d x / \pi^{n}$, where $d x$ denotes the $2 n$-dimensional Lebesgue measure. It is easily seen that the functions

$$
e_{j}(z)=z_{1}^{j_{1}} z_{2}^{j_{2}} \ldots z_{n}^{j_{n}} \equiv z^{j}, \quad z=\left(z_{1}, z_{2}, \ldots, z_{n}\right) \in \mathbf{C}^{n},
$$


where $j=\left(j_{1}, j_{2}, \ldots, j_{n}\right)$ is a multiindex, form a complete orthogonal basis in $A_{\alpha}^{2}$. By the familiar formula for the reproducing kernel [Berg, p. 8] we therefore have

$$
K_{\alpha}(x, \bar{y})=\sum_{j} \frac{e_{j}(x) \overline{e_{j}(y)}}{\left\|e_{j}\right\|_{\alpha}^{2}} .
$$

Since

$$
\left\|e_{j}\right\|_{\alpha}^{2}=\prod_{k=1}^{n} \int_{\mathbf{C}}\left|z_{k}\right|^{2 j_{k}} e^{-\alpha\left|z_{k}\right|^{2}} \frac{d z_{k}}{\pi}=\prod_{k=1}^{n} \int_{0}^{+\infty} t^{j_{k}} e^{-\alpha t} d t=\prod_{k=1}^{n} \frac{j_{k} !}{\alpha_{k}+1},
$$

we obtain

$$
K_{\alpha}(x, \bar{y})=\alpha^{n} \sum_{j} x^{j} \bar{y}^{j} \frac{\alpha^{|j|}}{j !}=\alpha^{n} e^{\alpha x \cdot \bar{y}} .
$$

Thus $K_{\alpha}(x, \bar{y}) a(x, \bar{y})^{\alpha}=\alpha^{n}$ and $\left(\mathrm{A}^{\prime}\right)$ is satisfied, with $c_{n}=1$ and $B(x, \bar{y})=$ $C(x, \bar{y}, \alpha)=0$. Finally,

$$
\frac{a(x, \bar{x}) a(y, \bar{y})}{|a(x, \bar{y})|^{2}}=e^{-\|x-y\|^{2}}
$$

so

$$
\psi(x \mid y)=-\|x-y\|^{2} \leq 0
$$

with equality iff $\|x-y\|=0$, i.e. iff $x=y$. It follows that all points of $\Omega$ are regular and so $\left(\mathrm{C}^{\prime}\right)$ holds. Theorem 2.12 therefore applies and yields a quantization procedure on $\mathbf{C}^{n}$.

It can be shown that this quantization, in fact, coincides with the familiar Weyl quantization on $\mathbf{C}^{n} \simeq \mathbf{R}^{2 n}$. See [Ber1], Section 4 , for details.

Example 2.14. Let $\Omega$ be a bounded symmetric domain in $\mathbf{C}^{n}$ in its HarishChandra realization, i.e. as a circular domain centered at the origin. It is well known that there exists a natural Kähler metric - the Bergman metric - on $\Omega$. Namely, let $A^{2}(\Omega)$ be the Hilbert space of all square-integrable (with respect to the Lebesgue measure) analytic functions on $\Omega$; its reproducing kernel $K(x, \bar{y})$ is the Bergman kernel function of $\Omega$. The form

$$
\omega=\sum_{j, k} \frac{\partial^{2} \log K(z, \bar{z})}{\partial z_{j} \partial \bar{z}_{k}} d z_{j} \wedge d \bar{z}_{k}
$$

is then a Kähler form on $\Omega$. It's clear that $\omega$ admits $\Psi(x)=\log K(x, \bar{x})$ as a potential, and thus $\left(\mathrm{E}^{\prime}\right)$ is satisfied with

$$
a(x, \bar{y})=1 / K(x, \bar{y}) \quad\left(\alpha_{0}=1\right) .
$$

It can be shown that, as in the preceding paragraph, $\left(\mathrm{A}^{\prime}\right)$ holds with $B(x, \bar{y})$ and $C(x, \bar{y}, \alpha)$ independent of $x$ and $y$ :

$$
K_{\alpha}(x, \bar{y}) a(x, \bar{y})^{\alpha}=\text { a polynomial (with constant coefficients) in } \alpha \text { of degree } n \text {. }
$$


(For a proof, see e.g. [FK1, p. 77], or Section 5 of [Ber1] or Chapter XIII.1 in the book [FK2].) It remains to check regularity. Observe that if $\Phi$ is a holomorphic automorphism of $\Omega$, then the well-known identity for the Bergman kernel function

$$
K(x, \bar{y})=K(\Phi(x), \overline{\Phi(y)}) \cdot J_{\Phi}(x) \overline{J_{\Phi}(y)}
$$

$\left(J_{\Phi}(x)=\right.$ the Jacobian of $\Phi$ at $\left.x\right)$ implies that

$$
\psi(\Phi(x) \mid \Phi(y))=\psi(x \mid y) \quad \forall \Phi \in \operatorname{Aut}(\Omega) .
$$

It follows that $y \in \Omega$ is regular if and only if $\Phi(y)$ is regular. As Aut $(\Omega)$ acts transitively on $\Omega$, it therefore suffices to show that the point $0 \in \Omega$ is regular. Since $\Omega$ is a circular domain, it follows from the mean value property of analytic functions and from the uniqueness of the reproducing kernel that

$$
K(\cdot, 0) \equiv \text { const. }(\neq 0)
$$

Thus

$$
\psi(x \mid 0)=\log \frac{K(0,0)}{K(x, \bar{x})} .
$$

Because the Bergman kernel is known to blow up at the boundary (i.e. $K(x, \bar{x}) \rightarrow$ $+\infty$ as $x \rightarrow \partial \Omega$ ), we conclude that $\psi(x \mid 0) \rightarrow-\infty$ as $x \rightarrow \partial \Omega$. Thus, to check regularity, it suffices to prove that $K(x, \bar{x})=K(0,0)$ implies $x=0$.

Suppose that $K(x, \bar{x})=K(0,0)$. In view of $(2.41)$,

$\|K(\cdot, \bar{x})-K(\cdot, 0)\|^{2}=K(x, \bar{x})-K(x, 0)-K(0, \bar{x})+K(0,0)=K(x, \bar{x})-K(0,0)=0$,

or $K(\cdot, \bar{x})=K(\cdot, 0)$. Thus $f(x)=f(0)$ for all $f \in A^{2}(\Omega)$. Since $\Omega$ is bounded, this holds, in particular, for all coordinate functions $f(z)=z_{j}(j=1,2, \ldots, n)$. Thus $x=0$ and we are done.

With $\left(\mathrm{A}^{\prime}\right),\left(\mathrm{C}^{\prime}\right)$ and $\left(\mathrm{E}^{\prime}\right)$ granted, Theorem 2.12 applies, and we obtain a quantization on bounded symmetric domains $\Omega$ with the Bergman metric.

Example 2.15. Let $\Omega$ be a planar domain of hyperbolic type, $\mathbf{D}$ the unit disc in $\mathbf{C}$ and $\phi: \mathbf{D} \rightarrow \Omega$ the uniformization map. Define the function $\lambda$ on $\Omega$ by

$$
\lambda(\phi(\xi))=\left(1-|\xi|^{2}\right) \cdot\left|\phi^{\prime}(\xi)\right|, \quad \xi \in \mathbf{D} .
$$

It's easy to see that the definition is correct. The Poincaré metric

$$
d s^{2}=\frac{2 d z d \bar{z}}{\lambda(z)^{2}}
$$

is then a Kähler metric on $\Omega$ of constant negative curvature. Hence the function

$$
\Psi(z)=-2 \log \lambda(z)
$$

is a choice for the Kähler potential on $\Omega$. The Bergman space $A_{\alpha}^{2}$ consists then of all analytic functions on $\Omega$ which satisfy

$$
\|f\|_{\alpha}^{2}=\frac{2}{\pi} \int_{\Omega}|f(x)|^{2} \lambda(x)^{2 \alpha-2} d x<+\infty,
$$


$d x$ being the (two-dimensional) Lebesgue measure on $\Omega$. These spaces are well known from the theory of automorphic functions [Kra].

Let us now consider our conditions $\left(\mathrm{E}^{\prime}\right),\left(\mathrm{A}^{\prime}\right)$ and $\left(\mathrm{C}^{\prime}\right)$. (2.42) suggests taking

$$
a(x, \bar{y})=(1-\xi \bar{\eta})^{2} \phi^{\prime}(\xi) \overline{\phi^{\prime}(\eta)}, \quad x=\phi(\xi), y=\phi(\eta) .
$$

This function, however, is not well-defined on $\Omega$ - the right-hand side makes sense only as a function on $\mathbf{D}$. We shall therefore "lift everything" to the universal cover, $\mathbf{D}$, of $\Omega$. Let

$$
G=\{\omega \in \operatorname{Aut}(\mathbf{D}): \phi \circ \omega=\omega\}
$$

be the covering group of $\phi$. Using the theory of automorphic functions, it can be shown that (cf. [E3], Section 2, where however the normalization was a different one, so $2 \alpha-1$ appears there instead of $\alpha-\frac{1}{2}$ here)

$$
K_{\alpha}(\phi(\xi), \overline{\phi(\eta)}) \cdot \phi^{\prime}(\xi)^{\alpha} \overline{\phi^{\prime}(\eta)^{\alpha}}=\left(\alpha-\frac{1}{2}\right) \sum_{\omega \in G}(1-\bar{\eta} \omega(\xi))^{-2 \alpha} \omega^{\prime}(\xi)^{\alpha} .
$$

Denote the RHS of $(2.43)$ by $a(\xi, \bar{\eta})$. Then

$$
\begin{aligned}
\frac{K_{\alpha}(\phi(\xi), \overline{\phi(\eta)}) a(\xi, \bar{\eta})^{\alpha}}{\alpha-\frac{1}{2}} & =(1-\xi \bar{\eta})^{2 \alpha} \sum_{\omega \in G}(1-\bar{\eta} \omega(\xi))^{-2 \alpha} \omega^{\prime}(\xi)^{\alpha} \\
& =\frac{(1-\xi \bar{\eta})^{2 \alpha}}{\left(1-|\xi|^{2}\right)^{\alpha}\left(1-|\eta|^{2}\right)^{\alpha}} \sum_{\omega \in G}\left(\omega_{\eta} \omega \omega_{-\xi}\right)^{\prime}(0)^{\alpha}
\end{aligned}
$$

where, for $a \in \mathbf{D}, \omega_{a} \in \operatorname{Aut}(\mathbf{D})$ is given by

$$
\omega_{a}(\xi)=\frac{\xi-a}{1-\bar{a} \xi}
$$

Let now $\mathcal{O}_{\eta} \subset \mathbf{D}$ be the fundamental domain for $G$ with center at $\eta$; that is,

$$
\mathcal{O}_{\eta}=\{\xi \in \mathbf{D}: d(\xi, \eta)<d(\xi, \omega(\eta)) \quad \forall \omega \in G, \omega \neq \text { id. }\}
$$

$d(\xi, \eta)=\left|\omega_{\xi}(\eta)\right|$ being the usual pseudohyperbolic distance on D. Since

$$
\begin{aligned}
\left|\left(\omega_{\eta} \omega \omega_{-\xi}\right)^{\prime}(0)\right| & =1-\left|\omega_{\eta} \omega \omega_{-\xi}(0)\right|^{2} \\
& =1-d(\eta, \omega(\xi))^{2}=1-d\left(\xi, \omega^{-1}(\eta)\right)^{2},
\end{aligned}
$$

it follows from the definition of $\mathcal{O}_{\eta}$ that

$$
\sum_{\omega \in G}\left(\omega_{\eta} \omega \omega_{-\xi}\right)^{\prime}(0)^{\alpha}=\left(\omega_{\eta} \omega_{-\xi}\right)^{\prime}(0)^{\alpha} \cdot\left[1+O\left(\gamma^{\alpha}\right)\right], \quad \forall \xi \in \mathcal{O}_{\eta},
$$

where $0 \leq \gamma=\gamma(\eta, \xi)<1$. Thus we finally arrive at

$$
\frac{K_{\alpha}(\phi(\xi), \overline{\phi(\eta)}) a(\xi, \bar{\eta})^{\alpha}}{\alpha-\frac{1}{2}}=1+O\left(\gamma^{\alpha}\right), \quad \forall \xi \in \mathcal{O}_{\eta} .
$$


Now a change of variable in the formula defining the Berezin transform

$$
B_{\alpha} f(y)=\int_{\Omega} f(x) \frac{\left|K_{\alpha}(x, \bar{y})\right|^{2}}{K_{\alpha}(y, \bar{y})} \lambda(x)^{2 \alpha-2} d x
$$

leads to

$$
B_{\alpha}(\phi(\eta))=\int_{\mathcal{O}_{\eta}} f(\phi(\xi)) \frac{\left|K_{\alpha}(\phi(\xi), \overline{\phi(\eta)})\right|^{2}}{K_{\alpha}(\phi(\eta), \overline{\phi(\eta)})} a(\xi, \bar{\xi})^{\alpha} \frac{d \xi}{\left(1-|\xi|^{2}\right)^{2}}
$$

But now we can forget completely about $\Omega$ and work only in the (simply connected!) fundamental domain $\mathcal{O}_{\eta}$. The function $a(\xi, \bar{\eta})$ can be used to settle $\left(\mathrm{E}^{\prime}\right),(2.44)$ is a substitute for $\left(\mathrm{A}^{\prime}\right)$, and the regularity $\left(\mathrm{C}^{\prime}\right)$ is a simple consequence of the fact that

$$
\frac{a(\xi, \bar{\xi}) a(\eta, \bar{\eta})}{|a(\xi, \bar{\eta})|^{2}}=\frac{\left(1-|\xi|^{2}\right)^{2}\left(1-|\eta|^{2}\right)^{2}}{|1-\xi \bar{\eta}|^{4}}=\left[1-d(\xi, \eta)^{2}\right]^{2} \leq 1 \quad \forall \xi, \eta \in \mathbf{D}
$$

with equality occurring if and only if $d(\xi, \eta)=0$, i.e. $\xi=\eta$.

True, the estimate $(2.44)$ is somewhat weaker than $\left(\mathrm{A}^{\prime}\right)$ or even $\left(\mathrm{A}^{\prime \prime}\right)$, but it was shown in [E3] that things can still be made to work even in such a case, and Theorem 2.10 can be obtained. Likewise, though (2.44) is too weak for a proof of Theorem 2.11 ("separation of points"), it can be used to give a proof of Theorem 2.11A when $\Omega$ is bounded. Thus, in the end, we see that the Berezin quantization can be carried out on all bounded planar domains of hyperbolic type with the Poincaré metric.

Example 2.16. Here we take as $\Omega$ the 1-dimensional cylinder, or, equivalently, the punctured complex plane $\mathbf{C} \backslash\{0\}$. The Kähler form on $\Omega$ is given by

$$
\omega=\frac{d z \wedge d \bar{z}}{z \bar{z}}
$$

This admits $\Psi(z)=\frac{1}{2} \log ^{2}(z \bar{z})$ as a Kähler potential. Polarization thus gives

$$
a(x, \bar{y})=\exp \left(-\frac{1}{2} \log ^{2} x \bar{y}\right)
$$

which, however, is not a well defined function on $\Omega$. As in the preceding example, we shall therefore pass to the universal covering surface (in this case, $\mathbf{C}$ ) of $\Omega$.

Prior to that, let us find the reproducing kernels $K_{\alpha}$. The Bergman space $A_{\alpha}^{2}$ consists of holomorphic functions in $\mathbf{C} \backslash\{0\}$ such that

$$
\|f\|_{\alpha}^{2}=\frac{1}{\pi} \int_{\mathbf{C} \backslash\{0\}}|f(x)|^{2} e^{-\frac{\alpha}{2} \log ^{2}|x|^{2}} \frac{d x}{x^{2}}<+\infty
$$

( $d x$ is the Lebesgue area measure). It's clear that the functions

$$
e_{j}(z)=z^{j}, \quad j \in \mathbf{Z}, z \in \mathbf{C} \backslash\{0\}
$$


are pairwise orthogonal elements of $A_{\alpha}^{2}$ (for each fixed $\alpha>0$ ). Since any function in $A_{\alpha}^{2}$ can be expanded into a Laurent series around 0 , it follows that the $e_{j}$ form, in fact, an orthogonal basis in $A_{\alpha}^{2}$. Thus

$$
K_{\alpha}(x, \bar{y})=\sum_{j \in \mathbf{Z}}\left\|e_{j}\right\|_{\alpha}^{-2} e_{j}(x) \overline{e_{j}(y)} .
$$

Computation gives

$$
\begin{aligned}
\left\|e_{k}\right\|_{\alpha}^{2} & =\int_{0}^{+\infty} r^{2 k} \exp \left(-\frac{\alpha}{2} \log ^{2} r^{2}\right) \frac{2 r d r}{r^{2}} \\
& =\int_{-\infty}^{+\infty} e^{k w-\frac{\alpha}{2} w^{2}} d w \\
& =\sqrt{\frac{2 \pi}{\alpha}} e^{k^{2} / 2 \alpha} .
\end{aligned}
$$

Therefore

$$
K_{\alpha}(x, \bar{y})=\sqrt{\frac{\alpha}{2 \pi}} \sum_{k \in \mathbf{Z}} e^{-k^{2} / 2 \alpha}(x \bar{y})^{k} .
$$

Now let $\xi \mapsto e^{\xi}$ be the covering map of $\mathbf{C}$ onto $\mathbf{C} \backslash\{0\}$ and denote $a(\xi, \bar{\eta})=$ $e^{-\frac{1}{2}(\xi+\bar{\eta})^{2}}$; this function, though not correctly defined on $\Omega \times \Omega$, is well-defined on $\mathbf{C} \times \mathbf{C}$. We have

$$
\begin{aligned}
K_{\alpha}\left(e^{\xi}, \overline{e^{\eta}}\right) a(\xi, \bar{\eta})^{\alpha} & =\sqrt{\frac{\alpha}{2 \pi}} \sum_{k} e^{-\frac{k^{2}}{2 \alpha}+k(\xi+\bar{\eta})-\frac{\alpha}{2}(\xi+\bar{\eta})^{2}} \\
& =\sqrt{\frac{\alpha}{2 \pi}} \sum_{k} \exp \left(-\frac{1}{2}\left[\sqrt{\alpha}(\xi+\bar{\eta})-\frac{k}{\sqrt{\alpha}}\right]^{2}\right) .
\end{aligned}
$$

By the Poisson summation formula,

$$
\sum_{k} \exp \left(-\frac{1}{2}\left[\sqrt{\alpha}(\xi+\bar{\eta})-\frac{k}{\sqrt{\alpha}}\right]^{2}\right)=\sqrt{2 \pi \alpha} \sum_{k} \exp \left[-2 \pi^{2} k^{2} \alpha+2 \pi i \alpha k(\xi+\bar{\eta})\right] .
$$

Thus

$$
\frac{K_{\alpha}\left(e^{\xi}, \overline{e^{\eta}}\right) a(\xi, \bar{\eta})^{\alpha}}{\alpha}=\sum_{k}\left(e^{-2 \pi^{2} k^{2}+2 \pi k i(\xi+\bar{\eta})}\right)^{\alpha} .
$$

Fix now $\eta \in \mathbf{C}, y=e^{\eta} \in \Omega$, and let $\mathcal{O}_{\eta}$ be the strip $\{\xi \in \mathbf{C}:|\operatorname{Im} \xi-\operatorname{Im} \eta|<\pi\}$. The mapping $\xi \mapsto e^{\xi}$ is then a bijection of $\mathcal{O}_{\eta}$ onto $\widetilde{\Omega} \subset \Omega$, where $\Omega \backslash \widetilde{\Omega}$ has measure zero. The Berezin transform on $\Omega$

$$
B_{\alpha} f(y)=\int_{\Omega} f(x) \frac{\left|K_{\alpha}(x, \bar{y})\right|^{2}}{K_{\alpha}(y, \bar{y})} e^{-\frac{\alpha}{2} \log ^{2}|x|^{2}} \frac{d x}{|x|^{2}}
$$

can be rewritten as

$$
B_{\alpha} f\left(e^{\eta}\right)=\int_{\mathcal{O}_{\eta}} f\left(e^{\xi}\right) \frac{\left|K_{\alpha}\left(e^{\xi}, \overline{e^{\eta}}\right)\right|^{2}}{K_{\alpha}\left(e^{\eta}, \overline{e^{\eta}}\right)} e^{-\frac{\alpha}{2}(\xi+\bar{\xi})^{2}} d \xi .
$$


Now, again, instead of quantizing $\Omega$, we can carry out the whole procedure in $\mathcal{O}_{\eta}$. The function $a(\xi, \bar{\eta})$ can be used to settle $\left(\mathrm{E}^{\prime}\right)$. The regularity assumption $\left(\mathrm{C}^{\prime}\right)$ is an immediate consequence of the fact that

$$
\frac{a(\xi, \bar{\xi}) a(\eta, \bar{\eta})}{|a(\xi, \bar{\eta})|^{2}}=e^{-|\xi-\eta|^{2}} \leq 1
$$

with equality occurring if and only if $\xi=\eta$. Finally, for $\xi \in \mathcal{O}_{\eta}$,

$$
|\operatorname{Re}[i(\xi+\bar{\eta})]|=|-\operatorname{Im}(\xi+\bar{\eta})|=|\operatorname{Im} \xi-\operatorname{Im} \eta|<\pi,
$$

whence

$$
\operatorname{Re}\left[-2 \pi^{2} k^{2}+2 \pi k i(\xi+\bar{\eta})\right]<-2 \pi^{2} k^{2}+2 \pi^{2} k=2 \pi^{2} k(1-k) \leq 0 \quad \text { for } k \neq 0,
$$

and it follows that the RHS of (2.46) is equal to $1+O\left(\gamma^{\alpha}\right)$, for some $\gamma \in(0,1)$; that is,

$$
\frac{K_{\alpha}\left(e^{\xi}, \overline{e^{\eta}}\right) a(\xi, \bar{\eta})^{\alpha}}{\alpha}=1+O\left(\gamma^{\alpha}\right) \quad \forall \xi \in \mathcal{O}_{\eta},
$$

where $0<\gamma=\gamma(\xi, \eta)<1$. This, again, is weaker than $\left(\mathrm{A}^{\prime \prime}\right)$, but, as in the preceding example, can be shown to be sufficient for establishing the analogue of Proposition 2.5 and hence of Theorem 2.10. We omit further details.

The potential $\Psi$ will certainly not exist globally in the case when the Kähler manifold $\Omega$ is compact. Moreover, in that case there are no nonconstant holomorphic functions on $\Omega$, so some further modifications are needed to define $A_{\alpha}^{2}$ in a sensible way - see e.g. [Ber2], Section 2. A different approach (considering sections of line bundles instead of functions) appears in [Pe1]; more recently, Berezin quantization on compact Kähler manifolds, and its connections with the geometric quantization of Kostant and Souriau, were investigated by Cahen, Gutt, and Rawnsley [CGR].

\section{Quantization on some Domains in $\mathbf{C}^{2}$}

In this section we shall apply the general procedure to a particular class of domains $\Omega \subset \mathbf{C}^{2}$ with a natural Kähler structure - namely, to pseudoconvex Reinhardt domains whose boundary enjoys certain smoothness and strong-pseudoconvexity properties.

Throughout this section, $F(t)$ will always stand for a non-increasing lower semicontinuous function from an interval $[0, B)$ into the extended positive reals $(0,+\infty]$ (the possibility $B=+\infty$ is not excluded). The Hartogs domain $\mathcal{D}_{F}$ corresponding to $F$ is, by definition,

$$
\mathcal{D}_{F}=\left\{x \in \mathbf{C}^{2}:\left|x_{1}\right|^{2}<B,\left|x_{2}\right|^{2}<F\left(\left|x_{1}\right|^{2}\right)\right\} .
$$

Recall that a domain $\Omega \subset \mathbf{C}^{2}$ is called Reinhardt if $y \equiv\left(y_{1}, y_{2}\right) \in \Omega$ whenever $x \equiv\left(x_{1}, x_{2}\right) \in \Omega$ and $\left|y_{1}\right|=\left|x_{1}\right|,\left|y_{2}\right|=\left|x_{2}\right|$. If the same holds even for all $y$ with $\left|y_{1}\right| \leq\left|x_{1}\right|$ and $\left|y_{2}\right| \leq\left|x_{2}\right|$, the Reinhardt domain is said to be complete. Clearly, the Hartogs domains $\mathcal{D}_{F}$ are complete Reinhardt domains. (The lower semicontinuity of $F$ ensures that $\mathcal{D}_{F}$ is an open set.) Conversely, it is easy to show that any complete Reinhardt domain is of the form $\mathcal{D}_{F}$ for some $F$. 
Throughout the rest of this section, we will be dealing exclusively with domains of the form $\mathcal{D}_{F}$. We shall first discuss how certain properties of $\mathcal{D}_{F}$ and of the natural Kähler structure on $\mathcal{D}_{F}$ (to be introduced below) can be expressed in terms of the function $F$. After that, we will deal with the quantization.

To each function $F$ we will associate a function $\phi=\phi_{F}$ by the recipe

$$
\phi(u)=\log F\left(e^{u}\right), \quad u \in(-\infty, \log B) .
$$

The function $\phi$ is a convenient means for describing when the domain $\mathcal{D}_{F}$ is pseudoconvex.

Proposition 3.1. If $\mathcal{D}_{F}$ is pseudoconvex, then either

(1) $F \equiv+\infty$ on $[0, B)$,

or

(2) $F$ is continuous on $[0, B)$, finite on $(0, B)$, and $\phi_{F}$ is concave on $(-\infty, \log B)$. Conversely, if $F$ satisfies (1) or (2), $\mathcal{D}_{F}$ is a pseudoconvex Reinhardt domain.

Proof. Being a complete Reinhardt domain, $\mathcal{D}_{F}$ is pseudoconvex iff it is logarithmically convex; the latter means that

$$
\log \mathcal{D}_{F}=\left\{(u, v) \in \mathbf{R}^{2}:\left(e^{u}, e^{v}\right) \in \mathcal{D}_{F}\right\}
$$

is a convex set in $\mathbf{R}^{2}$ [Hörm, Corollary 2.5.8]. In our case,

$$
\log \mathcal{D}_{F}=\left\{(u, v) \in \mathbf{R}^{2}: u<\log B, v<\phi(u)\right\}
$$

If $F \equiv+\infty$, then $\phi \equiv+\infty$ and $\log \mathcal{D}_{F}$ is a half-plane this corresponds to the case (1). Assume that $F\left(t_{0}\right)<+\infty$, whence also $\phi\left(t_{0}\right)<+\infty$, for some $t_{0}<B$ and $\log \mathcal{D}_{F}$ is convex. Let $p$ be a supporting line to $\log \mathcal{D}_{F}$ at the point $\left(t_{0}, \phi\left(t_{0}\right)\right)$. Then $p$ cannot be vertical (i.e. parallel to the $v$-axis) and the whole set $\log \mathcal{D}_{F}$ must lie below $p$. It follows that $\phi$ is finite everywhere, whence $F$ is finite on $(0, B)$. The convexity of $\log \mathcal{D}_{F}$ is then equivalent to $\phi$ being concave. Since a concave function on an interval is necessarily continuous, $\phi$ is continuous, and $F$ is continuous on $(0, B)$. As $F$ is non-increasing, we have $\lim _{t \rightarrow 0+} F(t) \leq F(0)$, and the lower semicontinuity of $F$ forces that equality must prevail. Thus, $F$ is even continuous on $[0, B)$. The converse part is obvious.

The case $F \equiv+\infty$ is of little interest (the corresponding $\mathcal{D}_{F}$ is biholomorphic to $\mathbf{D} \times \mathbf{C})$, and we will not consider it in the sequel.

Corollary 3.2. Assume that $F$ is continuous on $[0, B)$ and finite and $C^{2}$ on $(0, B)$. Then $\mathcal{D}_{F}$ is pseudoconvex iff $\left(t F^{\prime} / F\right)^{\prime} \leq 0$ on $(0, B)$.

Proof. The hypotheses imply that $\phi$ is finite and $C^{2}$ on $(-\infty, \log B)$. The concavity of $\phi$ is therefore equivalent to $\phi^{\prime \prime} \leq 0$. By (3.2),

$$
\phi^{\prime}(u)=\frac{d}{d u} \log F\left(e^{u}\right)=\frac{e^{u} F^{\prime}\left(e^{u}\right)}{F\left(e^{u}\right)},
$$

and

$$
\phi^{\prime \prime}(u)=\frac{d}{d u} \frac{e^{u} F^{\prime}\left(e^{u}\right)}{F\left(e^{u}\right)}=\left.\frac{d}{d t}\left(\frac{t F^{\prime}(t)}{F(t)}\right)\right|_{t=e^{u}} \cdot e^{u}
$$


Hence $\phi^{\prime \prime} \leq 0 \Longleftrightarrow\left(t F^{\prime} / F\right)^{\prime} \leq 0$, and the result follows from Proposition 3.1.

Recall that a real-valued function $\rho$ is called a defining function for $\Omega \subset \mathbf{C}^{n}$ at a point $z \in \partial \Omega$ if there exists a neighbourhood $U$ of $z$ such that

1. $\rho \in C^{2}(U)$

2. $\rho<0$ on $U \cap \Omega, \rho=0$ on $U \cap \partial \Omega$, and $\rho>0$ on $U \backslash \Omega$

3. $\operatorname{grad} \rho \neq 0$ on $\partial \Omega$.

The boundary $\partial \Omega$ is said to be strongly (or strictly) pseudoconvex at $z$ if the Levi form

$$
\mathcal{L}(\rho ; z)(X):=\sum_{j, k} \frac{\partial^{2} \rho(z)}{\partial z_{j} \partial \bar{z}_{k}} X_{j} \bar{X}_{k}, \quad X \in \mathbf{C}^{n}
$$

is positive for all non-zero vectors $X \in \mathbf{C}^{n}$ which satisfy

$$
\sum_{j} \frac{\partial \rho}{\partial z_{j}} X_{j}=0
$$

This definition is independent of the choice of the defining function $\rho$. For $\Omega=\mathcal{D}_{F}$, a characterization of strongly pseudoconvex boundary points is given in the next two propositions.

Proposition 3.3. Assume that $F$ is continuous on $[0, B)$ and finite and $C^{2}$ on $(0, B)$. Let $z \in \partial \mathcal{D}_{F}, 0<\left|z_{1}\right|^{2}<B$. Then $\partial \mathcal{D}_{F}$ is strongly pseudoconvex at the point $z \Longleftrightarrow\left(t F^{\prime} / F\right)^{\prime}<0$ at $t=\left|z_{1}\right|^{2}$.

Proof. The hypotheses imply that $\rho(z)=\left|z_{2}\right|^{2}-F\left(\left|z_{1}\right|^{2}\right)$ is a defining function for $\mathcal{D}_{F}$ at $z$. The Levi form (3.5) is given by

$$
\mathcal{L}(\rho ; z)(X)=\left|X_{2}\right|^{2}-\left(t F^{\prime}\right)^{\prime}\left(\left|z_{1}\right|^{2}\right)\left|X_{1}\right|^{2},
$$

and the condition $(3.6)$ reads

$$
\bar{z}_{1} F^{\prime}\left(\left|z_{1}\right|^{2}\right) X_{1}-\bar{z}_{2} X_{2}=0
$$

i.e. $X$ is a multiple of $\left(\bar{z}_{2}, \bar{z}_{1} F^{\prime}\left(\left|z_{1}\right|^{2}\right)\right)$. The positivity of $\mathcal{L}(\rho ; z)(X)$ is therefore equivalent to

$$
\left|z_{1}\right|^{2} F^{\prime}\left(\left|z_{1}\right|^{2}\right)^{2}-\left(t F^{\prime}\right)^{\prime}\left(\left|z_{1}\right|^{2}\right)\left|z_{2}\right|^{2}>0
$$

Since $\left|z_{2}\right|^{2}=F\left(\left|z_{1}\right|^{2}\right)$, this means

$$
t F^{\prime 2}-\left(t F^{\prime}\right)^{\prime} F>0
$$

or

$$
-F^{2} \cdot\left(\frac{t F^{\prime}}{F}\right)^{\prime}>0
$$

where $t=\left|z_{1}\right|^{2}$. Since $0<t<B$ and $F$ is finite and positive on $(0, B)$ by hypothesis, the result follows. 
Proposition 3.4. Assume that $F$ is continuous, finite and $C^{2}$ on $[0, B)$. Let $z$ be a point of $\mathcal{D}_{F}$ with $z_{1}=0$. Then $\partial \mathcal{D}_{F}$ is strongly pseudoconvex at $z \Longleftrightarrow$ $\left.\left(t F^{\prime} / F\right)^{\prime}\right|_{t=0}<0 \Longleftrightarrow F^{\prime}(0)<0$.

Proof. Under these hypotheses, the argument in the preceding proof applies also to the points $z \in \partial \mathcal{D}_{F}$ with $z_{1}=0$. The second equivalence is a consequence of the inequality

$$
\left.\left(\frac{t F^{\prime}}{F}\right)^{\prime}\right|_{t=0}=\frac{F^{\prime}(0)}{F(0)}
$$

Let us denote by $\mathfrak{B}$ the set of all pseudoconvex Reinhardt domains $\Omega \subset \mathbf{C}^{2}$ whose boundary is strongly pseudoconvex at all points $z \in \partial \Omega$ with $\left|z_{1}\right|^{2}<\sup _{x \in \Omega}\left|x_{1}\right|^{2}=$ $B$ and by $\mathfrak{F}$ the set of all non-increasing continuous functions $F(t)$ from an interval $[0, B)$ into $(0,+\infty]$ which are finite and $C^{2}$ on $\mathcal{I}$ and satisfy $\left(t F^{\prime} / F\right)^{\prime}<0$ for $t \in \mathcal{I}$, where $\mathcal{I}=(0, B)$ when $F(0)=+\infty$ and $\mathcal{I}=[0, B)$ when $F(0)<+\infty$. Our results so far can then be summarized as follows.

Theorem 3.5. $\mathfrak{B}=\left\{\mathcal{D}_{F} ; F \in \mathfrak{F}\right\}$.

Recall that a function $\Psi$ on a domain in $\mathbf{C}^{n}$ taking values in the interval $[-\infty,+\infty)$ is called plurisubharmonic $(P S H)$ if it is upper-semicontinuous and for each $v, x \in \mathbf{C}^{n}$, the function of one complex variable

$$
z \mapsto \Psi(x+z v)
$$

is subharmonic everywhere where it is defined. When $\Psi$ is $C^{2}$ on $\Omega$, then $\Psi$ is PSH on $\Omega$ iff its Levi (or Hessian) matrix

$$
\mathcal{L}(\Psi ; z)=\left(\frac{\partial^{2} \Psi(z)}{\partial z_{j} \partial \bar{z}_{k}}\right)_{j, k=1}^{n}
$$

is positive semidefinite: $\mathcal{L}(\Psi ; z) \geq 0 \forall z \in \Omega$. Alternatively, such a $\Psi$ is PSH on $\Omega$ iff it is a potential of a Riemannian pseudometric

$$
d s^{2}=\sum g_{j \bar{k}} d z_{j} d \bar{z}_{k}
$$

where

$$
g_{j \bar{k}}=\frac{\partial^{2} \Psi}{\partial z_{j} \partial \bar{z}_{k}}
$$

on $\Omega$. A function $\Psi$ which is $C^{2}$ on $\Omega$ and for which the Levi matrix $\mathcal{L}(\Psi ; z)$ is positive definite at each $z \in \Omega$ is said to be strictly plurisubharmonic $(s-P S H)$ on $\Omega$. This is equivalent to the Riemannian pseudometric (3.7) being nondegenerate: when $\Psi$ is s-PSH on all of $\Omega,(3.7)$ defines a Riemannian metric on $\Omega$.

Coming back to our domains $\Omega=\mathcal{D}_{F}$, introduce the functions

$$
a(z)=F\left(\left|z_{1}\right|^{2}\right)-\left|z_{2}\right|^{2}
$$


and

$$
\Psi(z)=\log \frac{1}{a(z)}
$$

on $\mathcal{D}_{F}$. We have $0<a \leq+\infty$ and $-\infty \leq \Psi<+\infty$. When $\Psi$ is s-PSH on $\Omega$ then, by what has just been said, (3.7) defines a natural Kähler metric on $\mathcal{D}_{F}$. The Kähler form is given by

$$
\omega_{F}=\sum_{j, k} g_{j \bar{k}} d z_{j} \wedge d \bar{z}_{k}
$$

where $g_{j \bar{k}}$ are given by (3.7).

It turns out that the (strict) plurisubharmonicity of $\Psi$ is closely related to the (strong) pseudoconvexity of $\mathcal{D}_{F}$. Before proving that, let us compute the elements of the corresponding Levi matrix $\mathcal{L}(\psi ; z)=\left(g_{j \bar{k}}\right)_{j, k=1}^{n}$ and its determinant (the Hessian ) $g=\operatorname{det}\left(g_{j \bar{k}}\right)$. We have

$$
\frac{\partial \Psi}{\partial z_{1}}=-\frac{1}{a} \cdot \frac{\partial a}{\partial z_{1}}=-\frac{1}{a} F^{\prime}\left(t_{1}\right) \bar{z}_{1}, \quad \frac{\partial \Psi}{\partial z_{2}}=-\frac{1}{a} \cdot \frac{\partial a}{\partial z_{2}}=\frac{\bar{z}_{2}}{a},
$$

where $t_{j}=\left|z_{j}\right|^{2}$. Therefore

and

$$
\begin{gathered}
g_{1 \overline{2}}=F^{\prime}\left(t_{1}\right) \bar{z}_{1} \frac{1}{a^{2}} \frac{\partial a}{\partial \bar{z}_{2}}=-\frac{\bar{z}_{1} z_{2} F^{\prime}\left(t_{1}\right)}{a^{2}}, \\
g_{2 \overline{1}}=\overline{g_{1} \overline{2}}=-\frac{z_{1} \bar{z}_{2} F^{\prime}\left(t_{1}\right)}{a^{2}} \quad \text { (by symmetry), } \\
g_{1 \overline{1}}=-\frac{F^{\prime}\left(t_{1}\right)}{a}-\frac{\bar{z}_{1}}{a} F^{\prime \prime}\left(t_{1}\right) z_{1}+F^{\prime}\left(t_{1}\right) \bar{z}_{1} \frac{1}{a^{2}} F^{\prime}\left(t_{1}\right) z_{1}, \\
g_{2 \overline{2}}=\frac{1}{a}-\frac{\bar{z}}{a^{2}}\left(-z_{2}\right)=\frac{a+t_{2}}{a^{2}}=\frac{F\left(t_{1}\right)}{a^{2}},
\end{gathered}
$$

The expression in the square brackets can be simplified to

$$
t_{1} F^{\prime 2} \cdot\left(F-t_{2}\right)-a F\left(F^{\prime}+t_{1} F^{\prime \prime}\right)=t_{1} F^{2} a-a F\left(t_{1} F^{\prime}\right)^{\prime}=-a F^{2} \cdot\left(\frac{t_{1} F^{\prime}}{F}\right)^{\prime},
$$

so we finally arrive at

$$
g=-\frac{F^{2}}{a^{3}} \cdot\left(\frac{t F^{\prime}}{F}\right)_{t=\left|z_{1}\right|^{2}}
$$

Observe that the normalized volume element corresponding to the metric (3.7) is thus given by

$$
d \mu(z)=\frac{F\left(\left|z_{1}\right|^{2}\right)^{2}}{a(z)^{3}} \cdot G\left(\left|z_{1}\right|^{2}\right) \frac{d z}{\pi^{2}}
$$

where $d z$ is the Lebesgue measure in $\mathbf{C}^{2}$ and

$$
G(t)=-\left(\frac{t F^{\prime}}{F}\right)^{\prime}
$$


Proposition 3.6. Assume that $F$ is continuous, finite and $C^{2}$ on $[0, B)$, and let $\Psi$ be given by (3.9). Then

1. $\Psi$ is $P S H$ on $\mathcal{D}_{F} \Longleftrightarrow\left(t F^{\prime} / F\right)^{\prime} \leq 0$ on $[0, B) \Longleftrightarrow \mathcal{D}_{F}$ is pseudoconvex,

2. $\Psi$ is s-PSH on $\mathcal{D}_{F} \Longleftrightarrow\left(t F^{\prime} / F\right)^{\prime}<0$ on $[0, B) \Longleftrightarrow \partial \mathcal{D}_{F}$ is strongly pseudoconvex at all points $z$ with $\left|z_{1}\right|^{2}<B$.

Proof. By the familiar criterion, the Levi matrix $\mathcal{L}(\Psi ; x)=\left(g_{j \bar{k}}\right)$ is positive definite if and only if

$$
g_{2 \overline{2}}(x)>0 \text { and } g(x)>0,
$$

and is positive semidefinite if and only if

$$
g_{2 \overline{2}}(x)>0 \text { and } g(x) \geq 0 .
$$

For bounded $F$ and $a$, the first halves of these conditions are always fulfilled by (3.15), while the second halves are in turn equivalent to

$$
\left(t F^{\prime} / F\right)^{\prime}<0 \text { and }\left(t F^{\prime} / F\right)^{\prime} \leq 0 \quad \text { at } t=\left|x_{1}\right|^{2}
$$

respectively, in view of (3.17). If we combine this with Propositions 3.2, 3.3 and 3.4 , the result follows immediately.

Lemma 3.7. Let $U$ be a neighbourhood of the origin in $\mathbf{C}$ and $h$ an upper semicontinuous function from $U$ into $\mathbf{R} \cup\{-\infty\}$ which is finite and subharmonic on $U \backslash\{0\}$ and satisfies $f(0)=-\infty$. Then $h$ is subharmonic on $U$.

Proof. We may assume that $U=$ D. Obviously,

$$
h(0)=-\infty \leq \frac{1}{\pi r_{0}^{2}} \int_{0}^{r_{0}} \int_{0}^{2 \pi} h\left(r e^{i \theta}\right) d \theta d r \quad \forall r_{0} \in(0,1),
$$

no matter what is the value of the double integral on the right-hand side. By [Hörm, Theorem 1.6.3], it follows that $h$ is subharmonic on all of $U$.

Proposition 3.8. Assume that $F$ is continuous on $[0, B)$, finite and $C^{2}$ on $(0, B)$, and $F(0)=+\infty$. Let $\Psi$ be given by (3.9) and denote $\widetilde{\mathcal{D}}=\left\{x \in \mathcal{D}_{F}, x_{1} \neq 0\right\}$. Then

1. $\Psi$ is $P S H$ on $\mathcal{D}_{F} \Longleftrightarrow\left(t F^{\prime} / F\right)^{\prime} \leq 0$ on $(0, B) \Longleftrightarrow \mathcal{D}_{F}$ is pseudoconvex,

2. $\Psi$ is s-PSH on $\widetilde{\mathcal{D}} \Longleftrightarrow\left(t F^{\prime} / F\right)^{\prime}<0$ on $(0, B) \Longleftrightarrow \partial \mathcal{D}_{F}$ is strongly pseudoconvex at all points $z$ with $\left|z_{1}\right|^{2}<B$.

Proof. Now things are complicated by the fact that $\Psi(x)=-\infty$ when $x_{1}=0$. (In particular, $\Psi$ is not $C^{2}$ at such points, and it does not make sense to speak of the strong-plurisubharmonicity of $\Psi$.) At all other points $\Psi$ is $C^{2}$, and the same argument as in the preceding proof shows that $\Psi$ is PSH on $\widetilde{\mathcal{D}}$ if and only if $\left(t F^{\prime} / F\right)^{\prime} \leq 0$ for $t \in(0, B)$, and s-PSH on $\widetilde{\mathcal{D}}$ iff $\left(t F^{\prime} / F\right)^{\prime}<0$ for $t \in(0, B)$; and, by Propositions 3.2 and 3.3 , these conditions are in turn equivalent to the indicated (strong) pseudoconvexity properties of the boundary $\partial \mathcal{D}_{F}$. To complete the proof, it therefore suffices to show that if $\Psi$ is PSH on $\widetilde{\mathcal{D}}$ then $\Psi$ is already PSH on all of $\mathcal{D}_{F}$.

To this aim, take $x \in \mathcal{D}_{F}$ with $x_{1}=0$ and $v \in \mathbf{C}^{n}$, and consider the function of one complex variable

$$
h(z)=\Psi(x+z v) .
$$


This function is defined on some neighbourhood $U$ of the origin and is continuous from $U$ into $\mathbf{R} \cup\{-\infty\}$. If $v_{1}=0$, then $h \equiv-\infty$, which is a subharmonic function. If $v_{1} \neq 0$, then $h$ is finite and subharmonic on $U \backslash\{0\}$ (since $\Psi$ is finite and PSH on $\widetilde{\mathcal{D}}$ ) and $h(0)=-\infty$. By the preceding lemma, $h$ is subharmonic on $U$. This completes the proof.

Let us now assume that the function $F(t)$, which has so far lived only on $[0, B)$, is actually defined on the whole disc $B \mathbf{D}=\{t \in \mathbf{C}:|t|<B\}$. The function $a(x)$ can then be extended to a function

$$
a(x, \bar{y})=F\left(x_{1} \bar{y}_{1}\right)-x_{2} \bar{y}_{2}
$$

on $\mathcal{D}_{F} \times \mathcal{D}_{F}$, whose restriction to the diagonal $x=y$ coincides with $a(x)$. Denote, as in Section 2,

$$
\psi(x \mid y)=\log \frac{a(x, \bar{x}) a(y, \bar{y})}{|a(x, \bar{y})|^{2}} .
$$

Recall that a point $y \in \mathcal{D}_{F}$ was called regular if $\psi(\cdot \mid y) \leq 0$ and

$$
\psi\left(x_{n} \mid y\right) \rightarrow 0 \Longrightarrow x_{n} \rightarrow y
$$

When $F(0)<+\infty$, we have seen that both the positive-definiteness of the Riemannian metric defined by the potential $\Psi$ and the strong pseudoconvexity of the boundary $\partial \mathcal{D}_{F}$ at all points $z$ with $\left|z_{1}\right|^{2}<B$ are equivalent to the inequality

$$
\left(t F^{\prime} / F\right)^{\prime}<0 \quad \text { on }[0, B) .
$$

It turns out that the regularity of all points of $\mathcal{D}_{F}$ also depends on this condition.

Proposition 3.9. Let $F$ be a function on BD taking values in $\mathbf{C} \cup\{\infty\}$ which is positive, finite, and $C^{2}$ on $[0, B)$, and let $a(x, \bar{y})$ be defined by (3.20). Then the following are equivalent:

(i) Each point $y \in \mathcal{D}_{F}$ is regular; that is,

$$
A(x \mid y) \stackrel{\text { def }}{=} \frac{a(x, \bar{x}) a(y, \bar{y})}{|a(x, \bar{y})|^{2}} \leq 1 \quad \forall x \in \mathcal{D}_{F},
$$

and $A\left(x_{n} \mid y\right) \rightarrow 1$ implies $x_{n} \rightarrow y$.

(ii) For all $x_{1}, y_{1} \in B^{1 / 2} \mathbf{D}$,

$$
F\left(x_{1} \mid y_{1}\right) \stackrel{\text { def }}{=} \frac{F\left(\left|x_{1}\right|^{2}\right) F\left(\left|y_{1}\right|^{2}\right)}{\left|F\left(x_{1} \bar{y}_{1}\right)\right|^{2}} \leq 1,
$$

and $F\left(x_{n, 1} \mid y_{1}\right) \rightarrow 1$ implies $x_{n, 1} \rightarrow y_{1}$.

(iii) $|F(t)| \geq F(|t|)$ for all $t \in B \mathbf{D}$, with equality iff $t \geq 0$, and $\left(t F^{\prime} / F\right)^{\prime}<0$ on $(0, B)$.

Proof. (i) $\Longrightarrow$ (ii). Immediate from $F\left(x_{1} \mid y_{1}\right)=A\left(\left(x_{1}, 0\right) \mid\left(y_{1}, 0\right)\right)$.

(ii) $\Longrightarrow$ (i). Denote

$$
w(x, \bar{y})=\frac{x_{2} \bar{y}_{2}}{F\left(x_{1} \bar{y}_{1}\right)}, \quad x, y \in \mathcal{D}_{F} .
$$


Then it follows from the definition of $A(x \mid y)$ and $F\left(x_{1} \mid y_{1}\right)$ that

$$
A(x \mid y)=F\left(x_{1} \mid y_{1}\right) \cdot \frac{(1-w(x, \bar{x}))(1-w(y, \bar{y}))}{|1-w(x, \bar{y})|^{2}} .
$$

By (3.22),

$$
|w(x, \bar{y})|^{2}=\frac{\left|x_{2}\right|^{2}\left|y_{2}\right|^{2}}{\left|F\left(x_{1} \bar{y}_{1}\right)\right|^{2}} \leq \frac{\left|x_{2}\right|^{2}}{F\left(\left|x_{1}\right|^{2}\right)} \frac{\left|y_{2}\right|^{2}}{F\left(\left|y_{1}\right|^{2}\right)}=w(x, \bar{x}) w(y, \bar{y}) \quad \forall x, y \in \mathcal{D}_{F} .
$$

Consequently,

$$
|1-w(x, \bar{y})| \geq 1-|w(x, \bar{y})| \geq 1-\sqrt{w(x, \bar{x}) w(y, \bar{y})} .
$$

Also, by the very definition of $\mathcal{D}_{F}$,

$$
w(x, \bar{x})=\frac{\left|x_{2}\right|^{2}}{F\left(\left|x_{1}\right|^{2}\right)} \in[0,1) \quad \forall x \in \mathcal{D}_{F} .
$$

A short computation therefore gives

$$
\frac{(1-w(x, \bar{x}))(1-w(y, \bar{y}))}{|1-\sqrt{w(x, \bar{x}) w(y, \bar{y})}|^{2}}=1-\left|\frac{\sqrt{w(x, \bar{x})}-\sqrt{w(y, \bar{y})}}{1-\sqrt{w(x, \bar{x}) w(y, \bar{y})}}\right|^{2} \leq 1,
$$

whence

$$
A(x \mid y) \leq F\left(x_{1} \mid y_{1}\right)\left[1-\left|\frac{\sqrt{w(x, \bar{x})}-\sqrt{w(y, \bar{y})}}{1-\sqrt{w(x, \bar{x}) w(y, \bar{y})}}\right|^{2}\right] \leq F\left(x_{1} \mid y_{1}\right) \leq 1,
$$

as asserted. Suppose now that $A\left(x_{n} \mid y\right) \rightarrow 1$. In view of the last formula, this implies that both $F\left(x_{n, 1} \mid y_{1}\right) \rightarrow 1$ and $w\left(x_{n}, \bar{x}_{n}\right) \rightarrow w(y, \bar{y})$. The former is equivalent to $x_{n, 1} \rightarrow y_{1}$ by hypothesis, and the latter then implies $\left|x_{n, 2}\right|^{2} \rightarrow\left|y_{2}\right|^{2}$. Thus, each cluster point $x$ of the sequence $x_{n}$ satisfies $x_{1}=y_{1}$ and $\left|x_{2}\right|=\left|y_{2}\right|$, i.e. lies in the interior of $\Omega$. By continuity, $A\left(x_{n} \mid y\right) \rightarrow 1$ implies $A(x \mid y)=1$. Using (3.25) once again, we see that

$$
F\left(x_{1} \mid y_{1}\right)=1, \quad w(x, \bar{x})=w(y, \bar{y}),
$$

and also equality must prevail in (3.24), which means that

$$
w(x, \bar{y}) \in[0,1]
$$

Since $x_{1}=y_{1}$ and $\left|x_{2}\right|=\left|y_{2}\right|$, the last condition implies that $x_{2} \bar{y}_{2} \geq 0$; thus $x=y$. We have shown that each cluster point of the sequence $x_{n}$ must coincide with $y$; but this means that $x_{n} \rightarrow y$.

(ii) $\Longrightarrow$ (iii). First, let $t \in B \mathbf{D}, t \neq 0$, and apply (3.22) to $y_{1}=|t|^{1 / 2}$ and $x_{1}=t|t|^{-1 / 2}$. This gives

$$
F\left(|t|^{2}\right) \leq|F(t)|^{2},
$$

with equality iff $x_{1}=y_{1}$, i.e. iff $t>0$. This proves the first part of (iii). Second, let $\phi=\phi_{F}$ be the function associated to $F$ by $(3.2)$, take $u, v \in(-\infty, \log B)$ and apply (3.22) to $x_{1}=e^{u / 2}$ and $y_{1}=e^{v / 2}$. This gives

$$
F\left(x_{1} y_{1}\right)^{2}=e^{2 \phi\left(\frac{u+v}{2}\right)} \geq F\left(\left|x_{1}\right|^{2}\right) F\left(\left|y_{1}\right|^{2}\right)=e^{\phi(u)+\phi(v)},
$$


or

$$
\phi\left(\frac{u+v}{2}\right) \geq \frac{\phi(u)+\phi(v)}{2},
$$

with equality iff $u=v$. In other words, $\phi$ is strictly concave, i.e. $\phi^{\prime \prime}<0$ on $(-\infty, \log B)$. By (3.4), this is equivalent to $\left(t F^{\prime} / F\right)^{\prime}<0$ on $(0, B)$.

(iii) $\Longrightarrow$ (ii). We shall temporarily extend the function $\phi$ and its derivative $\phi^{\prime}$ continuously to $[-\infty, \log B$ ) by setting (cf. (3.3))

$$
\phi(-\infty)=\log F(0), \quad \phi^{\prime}(-\infty)=0 .
$$

For $x_{1}, y_{1} \in B^{1 / 2} \mathbf{D}$ let $u, v \in[-\infty, \log B)$ be given by $u=\log \left|x_{1}\right|^{2}, v=\log \left|y_{1}\right|^{2}$. Then

$$
\begin{aligned}
F\left(x_{1} \mid y_{1}\right) & =\frac{F\left(\left|x_{1} \bar{y}_{1}\right|\right)^{2}}{\left|F\left(x_{1} \bar{y}_{1}\right)\right|^{2}} \cdot \frac{F\left(\left|x_{1}\right|^{2}\right) F\left(\left|y_{1}\right|^{2}\right)}{F\left(\left|x_{1} \bar{y}_{1}\right|\right)^{2}} \\
& =\frac{F\left(\left|x_{1} \bar{y}_{1}\right|\right)^{2}}{\left|F\left(x_{1} \bar{y}_{1}\right)\right|^{2}} \cdot e^{\phi(u)+\phi(v)-2 \phi\left(\frac{u+v}{2}\right)} .
\end{aligned}
$$

By hypothesis, $|F(t)| \geq F(|t|) \forall t \in B \mathbf{D}$ and $\phi$ is strictly concave; this implies that the last expression is less than or equal to one. Suppose now that $F\left(x_{n, 1} \mid y_{1}\right) \rightarrow 1$. Then necessarily

$$
\phi\left(u_{n}\right)+\phi(v)-2 \phi\left(\frac{u_{n}+v}{2}\right) \rightarrow 0
$$

and

$$
F\left(\left|x_{n, 1} \bar{y}_{1}\right|\right) /\left|F\left(x_{n, 1} \bar{y}_{1}\right)\right| \rightarrow 1
$$

Owing to the strict concavity of $\phi$,

$$
\frac{d}{d u}\left[\phi(u)+\phi(v)-2 \phi\left(\frac{u+v}{2}\right)\right]=\phi^{\prime}(u)-\phi^{\prime}\left(\frac{u+v}{2}\right)\left\{\begin{array}{l}
>0 \text { if } u<v \\
<0 \text { if } u>v
\end{array}\right.
$$

and, of course, $\phi(u)+\phi(v)-2 \phi\left(\frac{u+v}{2}\right)=0$ for $u=v$. Therefore (3.26) implies that $u_{n} \rightarrow v$, i.e. $\left|x_{n, 1}\right| \rightarrow\left|y_{1}\right|$. Thus each cluster point $x_{1}$ of the sequence $x_{n, 1}$ satisfies $\left|x_{1}\right|=\left|y_{1}\right|$, i.e. lies in the interior of $B \mathbf{D}$, and by (3.27) and continuity,

$$
F\left(\left|x_{1} \bar{y}_{1}\right|\right)=\left|F\left(x_{1} \bar{y}_{1}\right)\right| .
$$

By hypothesis, this means that $x_{1} \bar{y}_{1} \geq 0$, i.e. $x_{1}=y_{1}$. We have shown that each cluster point of the sequence $x_{n, 1}$ must coincide with $y_{1}$; but this means that $x_{n, 1} \rightarrow y_{1}$, q.e.d.

Now, following the Ansatz from Section 2, we will construct a quantization of $\left(\mathcal{D}_{F}, \omega_{F}\right)$. Thus, for the function $a(x, \bar{y})$ given by $(3.20)$ and the volume element given by (3.18), consider the Bergman space $A_{\alpha}^{2}$ of all holomorphic functions on $\Omega=\mathcal{D}_{F}$ square-integrable with respect to the measure

$$
a(z)^{\alpha} d \mu(z)=a(z)^{\alpha-3} F\left(\left|z_{1}\right|^{2}\right)^{2} G(z) \frac{d z}{\pi^{2}},
$$

$d z$ being the Lebesgue measure on $\mathbf{C}^{2}$. We will make the following assumptions on $F$ and $K_{\alpha}$. (The labelling (a)-(d) is not intended to correspond to $\left(\mathrm{A}^{\prime}\right)-\left(\mathrm{E}^{\prime}\right)$ of Section 2 or (A)-(D) in [Ber1].) Observe that, by virtue of Proposition 3.6, the first two of these assumptions ensure that $\partial \mathcal{D}_{F}$ is strongly pseudoconvex at all points $z$ with $z_{2} \neq 0$ and that $\omega$ is a Kähler metric on $\mathcal{D}_{F}$. 
Assumption (a). F is meromorphic on BD, with no poles on $[0, B)$.

Assumption (b). $F \in \mathfrak{F}$; that is (granted (a)), for $0 \leq t<B, F$ is finite and $F>0, F^{\prime}<0,\left(t F^{\prime} / F\right)^{\prime}<0$.

Assumption (c). $F(|t|) \leq|F(t)| \quad \forall t \in B \mathbf{D}$, with equality iff $t \geq 0$.

Assumption (d). There exists an infinite subset $E$ of the positive integers such that for all $\alpha \in E$,

$$
K_{\alpha}(x, \bar{y}) a(x, \bar{y})^{\alpha}=\alpha^{2}+B(x, \bar{y}) \alpha+C(x, \bar{y}, \alpha),
$$

where $B$ and $C$ are sesquianalytic in $x, y$ and bounded on $\Omega \times \Omega$ and $\Omega \times \Omega \times E$, respectively.

For each $\alpha \in E$, consider the algebras $\mathcal{A}_{h}$ of covariant symbols for $A_{\alpha}^{2}(h=1 / \alpha)$. Construct the algebra $\mathcal{A}$ and its linear subset $\widetilde{\mathcal{A}}$ as in Section 2. Then we have the following result.

Theorem 3.10. Suppose that (a)-(d) are fulfilled. Then the algebra $\mathcal{A}$ is a quantization of $\left(\mathcal{D}_{F}, \omega_{F}\right)$ satisfying the weak correspondence principle.

Proof. Owing to (a), the function $a(x, \bar{y})$ given by $(3.20)$ satisfies the condition $\left(\mathrm{E}^{\prime}\right)$ from Section 2 (with $\alpha_{0}=1$ ). By (b), (c) and Proposition 3.9, all points of $\mathcal{D}_{F}$ are regular, which is $\left(\mathrm{C}^{\prime}\right)$. Finally, $\left(\mathrm{A}^{\prime}\right)$ is immediate from $(\mathrm{d})$, and it only remains to apply Theorem 2.12 .

For practical purposes, we shall consider one more assumption. Denote

$$
c_{k}\left(F^{\alpha}\right)=\int_{0}^{B} t^{k} F(t)^{\alpha} G(t) d t
$$

where $G$ is given by the formula (3.19). The assumption is

Assumption $\left(\mathrm{d}^{\prime}\right)$. There exist an infinite subset $E$ of the positive integers and a real number $\gamma$ such that for all $\alpha \in E$,

$$
\sum_{k=0}^{\infty} t^{k} / c_{k}\left(F^{\alpha}\right)=(\alpha-1+\gamma) F(t)^{-\alpha} \quad \forall t \in B \mathbf{D} .
$$

Before clearing up the relation between $(d)$ and $\left(d^{\prime}\right)$, we derive some formulas for the reproducing kernels $K_{\alpha}$. The next proposition is probably well-known, but we give a proof here for completeness. ${ }^{7}$

Proposition 3.11. Let $\Omega$ be a complete Reinhardt domain in $\mathbf{C}^{2}$ and $\rho$ a positive locally integrable radial function on $\Omega$ (i.e. one depending only on $\left|z_{1}\right|$ and $\left|z_{2}\right|$ ) which is bounded away from zero on compact subsets of $\Omega$. Let $\mu$ be the measure $\rho(x) d x$, where $d x$ is the Lebesgue measure in $\mathbf{C}^{2}$. Then the evaluation functionals on the Bergman space $A^{2}(\Omega, \mu)$ are continuous and the reproducing kernel for $A^{2}(\Omega, \mu)$ is given by

$$
K(x, \bar{y})=\sum_{k, l \geq 0}\left(x_{1} \bar{y}_{1}\right)^{k}\left(x_{2} \bar{y}_{2}\right)^{l} /\left\|x_{1}^{k} x_{2}^{l}\right\|^{2}
$$

( with the convention that $1 /+\infty=0$ ).

\footnotetext{
${ }^{7}$ It has been called to the author's attention that another proof can be found in Skwarczynski $[\mathrm{Skw}]$
} 
Proof. The continuity of the evaluation functionals follows by the standard argument [Berg, p. 5] from the mean value property of analytic functions, the assumption that $\rho$ is bounded away from zero on compacts and locally integrable, and the Schwarz inequality. It remains to prove the formula (3.31).

Let $\mathcal{I} \subset \mathbf{N} \times \mathbf{N}(\mathbf{N}=$ nonnegative integers $)$ be the set of all indices $(k, l)$ for which $\left\|z_{1}^{k} z_{2}^{l}\right\|<+\infty$ and let $(k, l),\left(k_{1}, l_{1}\right) \in \mathcal{I}$. It's clear from the radiality of $\mu$ that $z_{1}^{k} z_{2}^{l} \perp z_{1}^{k_{1}} z_{2}^{l_{2}}$ unless $k=k_{1}$ and $l=l_{1}$. In other words, $z_{1}^{k} z_{2}^{l},(k, l) \in \mathcal{I}$, form an orthogonal system in $A^{2}$; we shall show that it is complete.

For $\theta, \sigma \in \mathbf{R}$, consider the operator

$$
U_{\theta, \sigma} f\left(\left(z_{1}, z_{2}\right)\right)=f\left(\left(e^{i \theta} z_{1}, e^{i \sigma} z_{2}\right)\right) .
$$

It is easy to see that $U_{\theta, \sigma}$ are unitary operators on $A^{2}$ and that, for each $f, U_{\theta, \sigma} f$ is a continuous $A^{2}$-valued function of $\theta$ and $\sigma$. It follows that for each $k, l \in \mathbf{N}$ the (Bochner) integral

$$
T_{k l} f=\frac{1}{4 \pi^{2}} \int_{0}^{2 \pi} \int_{0}^{2 \pi} e^{-k i \theta} e^{-l i \sigma} U_{\theta, \sigma} f d \theta d \sigma
$$

exists and defines a bounded linear operator $T_{k l}$ on $A^{2}$. As $U_{\theta, \sigma}^{*}=U_{-\theta,-\sigma}$ and as the $U$ 's clearly form a semigroup, it can be shown that the operators $T_{k l}$ satisfy

$$
T_{k l}^{*}=T_{k l} \quad \text { and } \quad T_{k l}^{2}=T_{k l}
$$

that is, they are projections on $A^{2}$ (possibly degenerating to zero operators). Let

$$
f(x)=\sum_{k, l \geq 0} f_{k l} x_{1}^{k} x_{2}^{l}
$$

be the Taylor expansion for $f \in A^{2}$ and let $P$ be a polydisc where (3.33) converges. In view of the continuity of the evaluation functionals, we have

$$
T_{k l} f(x)=\frac{1}{4 \pi^{2}} \int_{0}^{2 \pi} \int_{0}^{2 \pi} f\left(\left(e^{i \theta} x_{1}, e^{i \sigma} x_{2}\right)\right) e^{-k i \theta-l i \sigma} d \theta d \sigma=f_{k l} x_{1}^{k} x_{2}^{l}
$$

for each $x \in P$ - hence, for every $x \in \Omega$. Thus, $f_{k l} x_{1}^{k} x_{2}^{l} \in A^{2}$, and it follows that $T_{k l}=0$ for $(k, l) \notin \mathcal{I}$, while Range $\left(T_{k l}\right)=\mathbf{C} \cdot x_{1}^{k} x_{2}^{l}$ for $(k, l) \in \mathcal{I}$.

Now suppose that $f \in A^{2}$ is orthogonal to $x_{1}^{k} x_{2}^{l}$ for each $(k, l) \in \mathcal{I}$. Then $f$ is orthogonal to the range of each $T_{k l}$; hence, $T_{k l} f=0 \forall(k, l) \in \mathbf{N}$. By (3.34), $f_{k l}=0$ for all $k, l$, i.e. $f \equiv 0$. This proves the completeness of the orthogonal system $x_{1}^{k} x_{2}^{l}$, $(k, l) \in \mathcal{I}$.

The standard formula for the reproducing kernel [Berg, pp. 8-9] says that

$$
K(x, \bar{y})=\sum_{j} e_{j}(x) \overline{e_{j}(y)}
$$

where $\left\{e_{j}\right\}$ is any orthonormal basis of $A^{2}$. Using the orthogonal system obtained above, we get

$$
K(x, \bar{y})=\sum_{(k, l) \in \mathcal{I}}\left(x_{1} \bar{y}_{1}\right)^{k}\left(x_{2} \bar{y}_{2}\right)^{l} /\left\|x_{1}^{k} x_{2}^{l}\right\|^{2} .
$$

Granted the convention $1 /+\infty=0$, we can add the terms with $(k, l) \notin \mathcal{I}$ to the sum without doing any harm, and the required formula follows. 
Proposition 3.12. Let $F \in \mathfrak{F}$ (so that $\left.\mathcal{D}_{F} \in \mathfrak{B}\right)$. Then the reproducing kernel for the Bergman space $A_{\alpha}^{2}$ on $\mathcal{D}_{F}$ with respect to the measure (3.28) is given by

$$
K_{\alpha}(x, \bar{y})=(\alpha-2) \sum_{k, l \geq 0}\left(x_{1} \bar{y}_{1}\right)^{k}\left(x_{2} \bar{y}_{2}\right)^{l} \cdot\left(\begin{array}{c}
l+\alpha-2 \\
l
\end{array}\right) c_{k}\left(F^{l+\alpha}\right)^{-1} .
$$

Proof. Compute:

$$
\begin{aligned}
\left\|z_{1}^{k} z_{2}^{l}\right\|^{2} & =\int_{\Omega}\left|z_{1}^{k} z_{2}^{l}\right|^{2} a(z)^{\alpha} d \mu(z) \\
& =\int_{\Omega}\left|z_{1}^{k} z_{2}^{l}\right|^{2}\left[F\left(\left|z_{1}\right|^{2}\right)-\left|z_{2}\right|^{2}\right]^{\alpha-3} F\left(\left|z_{1}\right|^{2}\right)^{2} G\left(\left|z_{1}\right|^{2}\right) \frac{d z}{\pi^{2}} \\
& =\int_{0}^{B^{1 / 2}} \int_{0}^{F\left(r_{1}^{2}\right)^{1 / 2}} r_{1}^{2 k} r_{2}^{2 l}\left[F\left(r_{1}^{2}\right)-r_{2}^{2}\right]^{\alpha-3} F\left(r_{1}^{2}\right)^{2} G\left(r_{1}^{2}\right) 2 r_{2} d r_{2} 2 r_{1} d r_{1} \\
& =\int_{0}^{B} \int_{0}^{F\left(t_{1}\right)} t_{1}^{k} t_{2}^{l}\left[F\left(t_{1}\right)-t_{2}\right]^{\alpha-3} F\left(t_{1}\right)^{2} G\left(t_{1}\right) d t_{2} d t_{1} \\
& =\int_{0}^{B} \int_{0}^{1} t_{1}^{k} w^{l} F\left(t_{1}\right)^{l} \cdot F\left(t_{1}\right)^{\alpha-3}[1-w]^{\alpha-3} \cdot F\left(t_{1}\right)^{2} G\left(t_{1}\right) d w F\left(t_{1}\right) d t_{1} \\
& =\int_{0}^{1} w^{l}(1-w)^{\alpha-3} d w \cdot \int_{0}^{B} t_{1}^{k} F\left(t_{1}\right)^{l+\alpha} G\left(t_{1}\right) d t_{1} \\
& =\frac{l !(\alpha-3) !}{(l+\alpha-2) !} c_{k}\left(F^{l+\alpha}\right) .
\end{aligned}
$$

We have, in turn, used the formula (3.18) for $d \mu$; passed to the polar coordinates; made the substitution $r_{i}^{2}=t_{i}$; and made the substitution $t_{2}=w F\left(t_{1}\right)$. Substituting this into (3.31) gives the result.

We digress to make an interesting observation about the reproducing kernels.

Proposition 3.13. Denote, for a moment, $t_{i}=x_{i} \bar{y}_{i}$. Then

$$
\frac{1}{\alpha-2} \frac{d}{d t_{2}} K_{\alpha}=K_{\alpha+1}
$$

Proof. In view of (3.7), we have $K_{\alpha}(x, \bar{y})=(\alpha-2) \sum_{k, l \geq 0} t_{1}^{k} t_{2}^{l} / C_{k, l}(\alpha)$, where the numbers

$$
C_{k, l}(\alpha)=\frac{l !(\alpha-2) !}{(l+\alpha-2) !} c_{k}\left(F^{l+\alpha}\right)
$$

obey the recursion formula

$$
C_{k, l}(\alpha+1)=\frac{l !(\alpha-1) !}{(l+\alpha-1) !} c_{k}\left(F^{l+\alpha+1}\right)=\frac{\alpha-1}{l+1} C_{k, l+1}(\alpha) .
$$

Consequently,

$$
\begin{array}{r}
\frac{1}{\alpha-2} \frac{d}{d t_{2}} K_{\alpha}=\sum_{k, m=0}^{\infty} t_{1}^{k} t_{2}^{m-1} \cdot m / C_{k, m}(\alpha)=\sum_{k, l=0}^{\infty} t_{1}^{k} t_{2}^{l} \cdot(l+1) / C_{k, l+1}(\alpha) \\
=\sum_{k, l=0}^{\infty} t_{1}^{k} t_{2}^{l} \cdot(\alpha-1) / C_{k, l}(\alpha+1)=K_{\alpha+1},
\end{array}
$$

as asserted. 
For any $c \in \mathbf{C}$, the function ${ }^{8}$

$$
\begin{aligned}
H_{c}(x, \bar{y} ; \alpha) & =\frac{(\alpha-c-1) !}{(\alpha-3) !}\left(1-\frac{x_{2} \bar{y}_{2}}{F\left(x_{1} \bar{y}_{1}\right)}\right)^{c} a(x, \bar{y})^{-\alpha}, \quad x, y \in \mathcal{D}_{F} \\
& =\frac{(\alpha-c-1) !}{(\alpha-3) !}\left[1-t_{2} / F\left(t_{1}\right)\right]^{c} \cdot\left[F\left(t_{1}\right)-t_{2}\right]^{-\alpha}, \quad t_{i} \equiv x_{i} \bar{y}_{i}
\end{aligned}
$$

satisfies

$$
\frac{1}{\alpha-2} \frac{d}{d t_{2}} H_{c}(x, \bar{y} ; \alpha)=H_{c}(x, \bar{y} ; \alpha+1)
$$

i.e. $H_{c}$ is a solution to the recurrence relation (3.37). In all known cases when (d) holds, $K_{\alpha}$ is a (finite) linear combination of the functions $H_{c}$. It would be interesting to know if this fact generalizes in some way (perhaps with linear combinations replaced by infinite sums, etc.).

Returning to the main line of our argument, let us now investigate the consequences of the condition $\left(\mathrm{d}^{\prime}\right)$.

Proposition 3.14. Assume that $F \in \mathfrak{F}$ extends to a meromorphic function on $B \mathbf{D}$ and that $\left(\mathrm{d}^{\prime}\right)$ holds. Then the reproducing kernels for the spaces $A_{\alpha}^{2}$ on $\mathcal{D}_{F}$ are equal to

$$
K_{\alpha}(x, \bar{y})=(\alpha-2) a(x, \bar{y})^{-\alpha} \cdot[(\alpha-1)+\gamma(1-w)]
$$

where

$$
w=w(x, \bar{y})=\frac{x_{2} \bar{y}_{2}}{F\left(x_{1} \bar{y}_{1}\right)}
$$

Proof. Use $\left(\mathrm{d}^{\prime}\right)$ to carry out the summation over $k$ in (3.35). Denoting temporarily $x_{i} \bar{y}_{i}=t_{i}$, this gives

$$
\begin{aligned}
K_{\alpha}(x, \bar{y}) & =(\alpha-2) \sum_{l=0}^{\infty} t_{2}^{l}\left(\begin{array}{c}
l+\alpha-2 \\
l
\end{array}\right)(\alpha+l-1+\gamma) F\left(t_{1}\right)^{-l-\alpha} \\
& =(\alpha-2) \sum_{l=0}^{\infty}\left[(\alpha-1)\left(\begin{array}{c}
l+\alpha-1 \\
l
\end{array}\right)+\gamma\left(\begin{array}{c}
l+\alpha-2 \\
l
\end{array}\right)\right]\left[t_{2} / F\left(t_{1}\right)\right]^{l} F\left(t_{1}\right)^{-\alpha} \\
& =(\alpha-2) F\left(t_{1}\right)^{-\alpha}\left[(\alpha-1)\left(1-t_{2} / F\left(t_{1}\right)\right)^{-\alpha}+\gamma\left(1-t_{2} / F\left(t_{1}\right)\right)^{-\alpha+1}\right] \\
& =(\alpha-2)(\alpha-1) a(x, \bar{y})^{-\alpha}+\gamma(\alpha-2) a(x, \bar{y})^{-\alpha}\left[1-t_{2} / F\left(t_{1}\right)\right]
\end{aligned}
$$

which proves the formula (3.39).

\footnotetext{
${ }^{8}$ For $c$ non-integer, $(\alpha-c-1)$ ! is of course to be interpreted as $\Gamma(\alpha-c)$.
} 
Proposition 3.15. Assume that $F \in \mathfrak{F}$ extends to a meromorphic function on BD and that $\left(\mathrm{d}^{\prime}\right)$ holds. Then for each $\alpha \in E, \alpha>2, \alpha>1-\gamma$,

$$
\begin{gathered}
\mathbf{1} \in A_{\alpha}^{2}\left(\mathcal{D}_{F}\right) \Longleftrightarrow H^{\infty} \subset A_{\alpha}^{2}\left(\mathcal{D}_{F}\right) \Longleftrightarrow F(0)<+\infty, \\
x_{1} \in A_{\alpha}^{2}\left(\mathcal{D}_{F}\right) \Longleftrightarrow \lim _{t \rightarrow 0} \frac{-F^{\prime}(t)}{F(t)^{\alpha+1}}>0 .
\end{gathered}
$$

(The limit always exists and is nonnegative.) Here $x_{1}$ stands for the first coordinate function.

Proof. It's clear that $H^{\infty} \subset A_{\alpha}^{2} \Longleftrightarrow \mathbf{1} \in A_{\alpha}^{2}$. By (3.36),

$$
\|\mathbf{1}\|_{\alpha}^{2}=\frac{1}{\alpha-2} c_{0}\left(F^{\alpha}\right)
$$

By $\left(d^{\prime}\right)$,

$$
(\alpha+\gamma-1) F(0)^{-\alpha}=\sum_{k=0}^{\infty} t^{k} /\left.c_{k}\left(F^{\alpha}\right)\right|_{t=0}=1 / c_{0}\left(F^{\alpha}\right)
$$

or

$$
c_{0}\left(F^{\alpha}\right)=(\alpha+\gamma-1)^{-1} F(0)^{\alpha},
$$

and the first assertion follows. Similarly, from $\left(\mathrm{d}^{\prime}\right)$ we have

$$
1 / c_{1}\left(F^{\alpha}\right)=(\alpha+\gamma-1) \lim _{t \rightarrow 0} \frac{F(t)^{-\alpha}-F(0)^{-\alpha}}{t}=(\alpha+\gamma-1) \lim _{t \rightarrow 0} \frac{-\alpha F^{\prime}(t)}{F(t)^{\alpha+1}}
$$

(by the l'Hospital rule). Hence by (3.36),

$$
\left\|x_{1}\right\|_{\alpha}^{2}=\frac{1}{\alpha-2} c_{1}\left(F^{\alpha}\right)<+\infty \Longleftrightarrow 1 / c_{1}\left(F^{\alpha}\right)>0 \Longleftrightarrow \lim _{t \rightarrow 0} \frac{-F^{\prime}(t)}{F(t)^{\alpha+1}}>0,
$$

which gives the second assertion.

Proposition 3.16. Assume that (a), (b) and ( $\left.\mathrm{d}^{\prime}\right)$ are fulfilled. Then so is (c).

Proof. Since the hypotheses of Proposition 3.14 are satisfied, we can apply the formula (3.39) to $y=\left(|t|^{1 / 2}, 0\right), x=\left(t|t|^{-1 / 2}, 0\right)$, where $0 \neq t \in B \mathbf{D}$. Since all the $w$ 's vanish, we get

$$
\frac{\left|K_{\alpha}(x, \bar{y})\right|^{2}}{K_{\alpha}(x, \bar{x}) K_{\alpha}(y, \bar{y})}=\left[\frac{F\left(\left|x_{1}\right|^{2}\right) F\left(\left|y_{1}\right|^{2}\right)}{\left|F\left(x_{1} \bar{y}_{1}\right)\right|^{2}}\right]^{\alpha}=\left[\frac{F(|t|)}{|F(t)|}\right]^{2 \alpha} .
$$

As the LHS does not exceed one by the Schwarz inequality, the same is true for the RHS, and we conclude that

$$
F(|t|) \leq|F(t)|
$$

Suppose that equality occurs for some $t \neq 0$. Let $z=|t|^{1 / 2}$ and $\epsilon=t /|t|$. Tracing back, we see that there exist two points $y=(z, 0)$ and $x=(\epsilon z, 0)$ such that $K_{\alpha}(\cdot, \bar{x})$ 
and $K_{\alpha}(\cdot, \bar{y})$ are linearly dependent. This means that there exist $a, b \in \mathbf{C}$, not both zero, such that

$$
a f(x)+b f(y)=0 \quad \forall f \in A_{\alpha}^{2} .
$$

Now owing to (a) and (b) we have $F(0)<+\infty$ and

$$
\frac{F^{\prime}(0)}{F(0)}=\left.\left(\frac{t F^{\prime}}{F}\right)^{\prime}\right|_{t=0}<0
$$

By Proposition 3.15 it follows that $1 \in A_{\alpha}^{2}$ and $x_{1} \in A_{\alpha}^{2}$. Thus,

$$
a+b=0, \quad a z+b \epsilon z=0,
$$

or $z=\epsilon z$ and $t=|t|$. This means that equality can occur in (3.42) only when $t \geq 0$, and $(\mathrm{c})$ is proved.

Corollary 3.17. Assume that (a), (b) and ( $\left.\mathrm{d}^{\prime}\right)$ hold. Then so does (d).

Proof. It follows from Proposition 3.9 (ii) (or from the first equality in (3.41) above) that

$$
F\left(\left|x_{1}\right|^{2}\right) F\left(\left|y_{1}\right|^{2}\right) \leq\left|F\left(x_{1} \bar{y}_{1}\right)\right|^{2}, \quad \forall x, y \in \mathcal{D}_{F} .
$$

Thus

$$
|w(x, \bar{y})|^{2}=\frac{\left|x_{2}\right|^{2}\left|y_{2}\right|^{2}}{\left|F\left(x_{1} \bar{y}_{1}\right)\right|^{2}} \leq \frac{\left|x_{2}\right|^{2}}{F\left(\left|x_{1}\right|^{2}\right)} \cdot \frac{\left|y_{2}\right|^{2}}{F\left(\left|y_{1}\right|^{2}\right)}<1
$$

by the very definition of $\mathcal{D}_{F}$. That is,

$$
w(x, \bar{y}) \in \mathbf{D} \quad \forall x, y \in \mathcal{D}_{F} .
$$

Inserting this into (3.39), we get (d) at once, with $C(x, \bar{y}, \alpha)=2-2 \gamma(1-w)$ and $B(x, \bar{y})=\gamma(1-w)-3$.

Theorem 3.18. Assume that (a), (b) and $\left(\mathrm{d}^{\prime}\right)$ are fulfilled. Then the algebra $\mathcal{A}$ is a quantization of $\left(\mathcal{D}_{F}, \omega_{F}\right)$ satisfying the weak correspondence principle.

Proof. Proposition 3.16, Corollary 3.17, and Theorem 3.10.

Let us now (at last!) give some more examples of the Berezin quantization. In all of them, we will be considering $\Omega=\mathcal{D}_{F}$ with the Kähler form $\omega=\omega_{F}$ (3.10) for some function $F \in \mathfrak{F}$.

Example 3.19. $F(t)=(1-t)^{p}, p>0 ; B=1$. The domains $\mathcal{D}_{F}$ can then be written as

$$
\mathcal{D}_{F}=\left\{z \in \mathbf{C}^{2}:\left|z_{1}\right|^{2}+\left|z_{2}\right|^{2 / p}<1\right\}
$$

and represent a special case of the complex ellipsoids (or Thullen domains). (a) is clearly satisfied $-F \in \mathcal{O}(\mathbf{D})$. As for (b), we have $F(t)>0$,

$$
F^{\prime}(t)=-p(1-t)^{p-1}<0, \quad\left(\frac{t F^{\prime}}{F}\right)^{\prime}=-\frac{p}{(1-t)^{2}}<0
$$


on $[0,1)$, so (b) holds. (c) follows from the triangle inequality $|1-t| \geq 1-|t|$, but we won't actually need it, since we are going to show that $\left(\mathrm{d}^{\prime}\right)$ holds. By (3.29),

$$
\begin{aligned}
c_{k}\left(F^{\alpha}\right) & =\int_{0}^{1} t^{k}(1-t)^{\alpha p} \cdot \frac{p}{(1-t)^{2}} d t \\
& =p \cdot \frac{k !(\alpha p-2) !}{(k+\alpha p-1) !} .
\end{aligned}
$$

Consequently,

$$
\begin{aligned}
\sum_{k=0}^{\infty} t^{k} / c_{k}\left(F^{\alpha}\right) & =\frac{\alpha p-1}{p} \sum_{k=0}^{\infty}\left(\begin{array}{c}
k+\alpha p-1 \\
k
\end{array}\right) t^{k} \\
& =\left(\alpha-\frac{1}{p}\right) \cdot(1-t)^{-\alpha p} \\
& =\left(\alpha-\frac{1}{p}\right) F(t)^{-\alpha},
\end{aligned}
$$

so $\left(\mathrm{d}^{\prime}\right)$ is satisfied with $\gamma=1-\frac{1}{p}$. Thus, Theorem 3.18 applies and yields a quantization on the complex ellipsoids $\mathcal{D}_{F}$.

The reproducing kernels are, by (3.39), given by

$$
K_{\alpha}(x, \bar{y})=(\alpha-2)\left[(\alpha-1)+\left(1-\frac{1}{p}\right)\left(1-\frac{t_{2}}{\left(1-t_{1}\right)^{p}}\right)\right] \cdot\left[\left(1-t_{1}\right)^{p}-t_{2}\right]^{-\alpha}
$$

where, as usual, $t_{i}=x_{i} \bar{y}_{i}$. This formula has recently been obtained by Liu and Stoll [LS].

For $p=1, \mathcal{D}_{F}$ is just the unit ball $\mathbf{B}_{2}$ in $\mathbf{C}^{2}$, and

$$
K_{\alpha}(x, \bar{y})=(\alpha-2)(\alpha-1)\left(1-x_{1} \bar{y}_{1}-x_{2} \bar{y}_{2}\right)^{-\alpha}
$$

is the familiar "weighted" Bergman kernel for $\mathbf{B}_{2}$.

Example 3.20. $F(t)=e^{-t} ; B=+\infty$. This domain was considered by Springer [Spr]. As $F$ is an entire function, (a) is satisfied. For $t>0, F(t)>0, F^{\prime}(t)=$ $-F(t)<0$, and

$$
\left(t F^{\prime} / F\right)^{\prime}=-1<0
$$

so (b) holds. (c) follows from the fact that $|t| \geq \operatorname{Re} t$, but, again, need not be checked because we are going to show that $\left(\mathrm{d}^{\prime}\right)$ holds. By (3.29),

$$
c_{k}\left(F^{\alpha}\right)=\int_{0}^{+\infty} t^{k} e^{-\alpha t} d t=\frac{k !}{\alpha^{k+1}}
$$

whence

$$
\sum_{k=0}^{\infty} \frac{t^{k}}{c_{k}\left(F^{\alpha}\right)}=\alpha \sum_{k=0}^{\infty} \frac{(\alpha t)^{k}}{k !}=\alpha e^{\alpha t}=\alpha F^{-\alpha}
$$

and $\left(\mathrm{d}^{\prime}\right)$ is satisfied with $\gamma=1$. By Theorem 3.18, Berezin quantization can be carried out on $\mathcal{D}_{F}$.

The reproducing kernels for $A_{\alpha}^{2}$ are

$$
K_{\alpha}(x, \bar{y})=(\alpha-2)\left[e^{-x_{1} \bar{y}_{1}}-x_{2} \bar{y}_{2}\right]^{-\alpha} \cdot\left[(\alpha-1)+\left(1-x_{2} \bar{y}_{2} e^{x_{1} \bar{y}_{1}}\right)\right] .
$$


Example 3.21. $F(t)=c /(t+c), c>0 ; B=+\infty$. Again, (a) is clear, and (b) follows from

$$
F(t)>0, \quad F^{\prime}(t)=\frac{-c}{(t+c)^{2}}<0, \quad\left(\frac{t F^{\prime}}{F}\right)^{\prime}=\frac{-c}{(t+c)^{2}}<0
$$

for $0 \leq t<+\infty$. (c) can either be inferred from $\left(\mathrm{d}^{\prime}\right)$, or verified directly by the triangle inequality. As for $\left(\mathrm{d}^{\prime}\right)$, we have, by (3.29),

$$
c_{k}\left(F^{\alpha}\right)=\int_{0}^{+\infty} t^{k} \frac{c^{\alpha}}{(t+c)^{\alpha}} \cdot \frac{c}{(t+c)^{2}} d t= \begin{cases}+\infty & \text { if } k>\alpha, \\ \frac{k !(\alpha-k) !}{(\alpha+1) !} c^{k} & \text { if } 0 \leq k \leq \alpha .\end{cases}
$$

Thus,

$$
\sum_{k=0}^{\infty} t^{k} / c_{k}\left(F^{\alpha}\right)=\sum_{k=0}^{\alpha}(\alpha+1)\left(\begin{array}{l}
\alpha \\
k
\end{array}\right) t^{k} c^{-k}=(\alpha+1)\left(1+\frac{t}{c}\right)^{\alpha}=(\alpha+1) F^{-\alpha}
$$

and $\left(\mathrm{d}^{\prime}\right)$ holds with $\gamma=2$. Hence, $\left(\mathcal{D}_{F}, \omega_{F}\right)$ is Berezin quantizable.

The reproducing kernels are given by

$$
K_{\alpha}(x, \bar{y})=(\alpha-2)\left[(\alpha-1)+2\left(1-x_{2} \bar{y}_{2}\left(x_{1} \bar{y}_{1}+c\right) / c\right)\right] \cdot\left[\frac{c}{x_{1} \bar{y}_{1}+c}-x_{2} \bar{y}_{2}\right]^{-\alpha} \text {. }
$$

Remark 3.22. Let $\alpha>0, \beta>0, g \in A_{\beta}^{2}$, and consider the integral

$$
\int_{\Omega}|g(z)|^{2} \cdot|a(z, \bar{v})|^{2 \beta} \cdot a(z, \bar{z})^{\alpha} d \mu(z)
$$

with $\Omega=\mathcal{D}_{F}, F(t)=1 /(t+1)$, as above (for $c=1$ ) and $v \in \Omega$. Let $x \in \Omega$ be such that $x_{1} \neq 0$ and $g(x) \neq 0$, and take $v=\left(-1 / \bar{x}_{1}, 0\right)$. If $U$ is a small neighbourhood of $x$, then $|g(z)| \simeq|g(x)|>0$ etc. for $z \in U$, and $|a(z, \bar{v})|=\left|1-z_{1} / x_{1}\right|^{-1}$, so

$$
\begin{aligned}
\int_{U}|g(z)|^{2}|a(z, \bar{v})|^{2 \beta} & a(z, \bar{z})^{\alpha} d \mu(z) \\
& \simeq|g(x)|^{2} a(x, \bar{x})^{\alpha} \frac{F\left(\left|x_{1}\right|^{2}\right)^{2} G\left(\left|x_{1}\right|^{2}\right)}{a(x, \bar{x})^{3}}\left|x_{1}\right|^{2 \beta} \int_{U}\left|z_{1}-x_{1}\right|^{-2 \beta} d z \\
& \simeq \text { const. } \cdot \int_{0}^{\epsilon} t^{-\beta} d t=+\infty \quad \text { if } \beta \geq 1
\end{aligned}
$$

Thus there always exists $v \in \Omega$ such that the integral (3.44) is infinite for all $\alpha>0$, unless $g \equiv 0$ or $\beta<1$. If $\beta<1$, then $A_{\beta}^{2}=\{0\}$ in view of (3.36), so $g \equiv 0$ in either case.

In particular, if $g \in A_{\beta}^{2}$ separates two points of $\Omega$, then there always exists $v \in \Omega$ such that the integral (3.44) is infinite for any $\alpha>0$. This invalidates the argument in the (end of the) proof of Theorem 2.3 of [Ber1]. See the footnote ${ }^{5}$ before Theorem 2.10 above. 
Example 3.23. $F(t)=1 /(t+1)^{p}, p$ a positive integer; $B=+\infty$. Once more (a) is obvious, and

$$
F^{\prime}(t)=-p(t+1)^{-p-1}<0, \quad\left(\frac{t F^{\prime}}{F}\right)^{\prime}=\frac{-p}{(t+1)^{2}}<0 \quad \forall t \geq 0
$$

imply (b). (c) follows either from the triangle inequality or from $\left(d^{\prime}\right)$. As for $\left(d^{\prime}\right)$, we have, by a computation analogous to the one in the preceding example,

$$
c_{k}\left(F^{\alpha}\right)=p \cdot \frac{k !(\alpha p-k) !}{(\alpha p+1) !} \quad \text { for } 0 \leq k<\alpha p
$$

and equals $+\infty$ otherwise. Hence,

$$
\sum_{k=0}^{\infty} t^{k} / c_{k}\left(F^{\alpha}\right)=\frac{\alpha p+1}{p} \sum_{k=0}^{\alpha p}\left(\begin{array}{c}
\alpha p \\
k
\end{array}\right) t^{k}=\left(\alpha+\frac{1}{p}\right)(1+t)^{\alpha p}=\left(\alpha+\frac{1}{p}\right) F^{-\alpha},
$$

and $\left(\mathrm{d}^{\prime}\right)$ holds with $\gamma=1+\frac{1}{p}$. Thus $\left(\mathcal{D}_{F}, \omega_{F}\right)$ is quantizable.

The reproducing kernels are given by

$$
K_{\alpha}(x, \bar{y})=(\alpha-2)\left[(\alpha-1)+(1+1 / p)\left(1-x_{2} \bar{y}_{2}\left(1+x_{1} \bar{y}_{1}\right)^{p}\right)\right] \cdot\left[\left(1+x_{1} \bar{y}_{1}\right)^{-p}-x_{2} \bar{y}_{2}\right]^{-\alpha} .
$$

We close this section by an example of a different type. So far, we have always been able to compute the reproducing kernels explicitly, and they possessed the required asymptotic behaviour - that is, (d) was satisfied. The next example shows that (d) can break down, too. Let us first put down the following simple consequence of Proposition 2.1.

Proposition 3.24. Let $F$ be a function on BD taking values in $\mathbf{C} \cup\{\infty\}$ which is finite, positive and continuous on $[0, B)$, and let $a(x, \bar{y})$ be given by (3.20). Suppose that (d) holds. Then $|F(t)| \geq F(|t|)$ for all $t \in B \mathbf{D}$.

Proof. By Proposition 2.1, (d) implies that

$$
\frac{a(x, \bar{x}) a(y, \bar{y})}{|a(x, \bar{y})|^{2}} \leq 1 \quad \forall x, y \in \mathcal{D}_{F} .
$$

Take $y=\left(|t|^{1 / 2}, 0\right), x=\left(t|t|^{-1 / 2}, 0\right)$, where $0 \neq t \in B \mathbf{D}$. The last inequality then becomes

$$
\frac{F(|t|) F(|t|)}{|F(t)|^{2}} \leq 1
$$

and the assertion follows.

Example 3.25. $F(t)=t^{3}-3 t^{2}-\frac{1}{16} t+\frac{33}{16}, B=1$. Since $F$ is a polynomial, (a) obviously holds. For $t \in[0,1)$, we have

$$
\begin{aligned}
F(t) & =(t-1)\left(t+\frac{3}{4}\right)\left(t-\frac{11}{4}\right)>0 \\
F^{\prime}(t) & =3 t(t-2)-\frac{1}{16} \leq-\frac{1}{16}<0 \\
\frac{t F^{\prime}}{F} & =3+\frac{3 t^{2}+\frac{1}{8} t-\frac{99}{16}}{t^{3}-3 t^{2}-\frac{1}{16} t+\frac{33}{16}}
\end{aligned}
$$


and

$$
\left(\frac{t F^{\prime}}{F}\right)^{\prime}=\frac{\left(6 t+\frac{1}{8}\right)\left(t^{3}-3 t^{2}-\frac{1}{16} t+\frac{33}{16}\right)-\left(3 t^{2}+\frac{1}{8} t-\frac{99}{16}\right)\left(3 t^{2}-6 t-\frac{1}{16}\right)}{F(t)^{2}}<0
$$

because the numerator is equal to

$-3 t^{4}-\frac{1}{4} t^{3}+\frac{75}{4} t^{2}-\frac{99}{4} t-\frac{33}{256}=-3 t^{4}-\frac{1}{4} t^{3}+\frac{75}{4}\left(t^{2}-t\right)-6 t-\frac{33}{256} \leq-\frac{33}{256}<0$.

Thus, (b) is also fulfilled. On the other hand,

$$
|F(-3 / 4)|=0<F(3 / 4)=3 / 4
$$

and Proposition 3.24 implies that (d) cannot be satisfied. Thus we have an example of a pseudoconvex domain $\mathcal{D}_{F}$ for which (a) and (b) hold, whereas (c) and (d) do not; of course, there is no hope of performing Berezin quantization in this case.

\section{Appendix. Some other examples}

Let us briefly deal with the situation when $F$ behaves "badly" at the origin that is, when $F^{\prime}(0)$ vanishes, or when $F(0)=+\infty$ (so the Hartogs domain $\mathcal{D}_{F}$ is unbounded in the $z_{2}$ variable). The form $\omega_{F}$ then does not define a Kähler metric — that is, it either degenerates (i.e. one has only a pseudometric, not a metric the corresponding quadratic form is not positive definite) or has "cusp" singularities (blows up at points with $z_{1}=0$ ). For this reason, we have relegated this discussion to a separate appendix. On the other hand, it still makes perfectly good sense to investigate the validity of (2.17), which is of some interest in its own right. Besides, we obtain several examples which illustrate the limitations of our method and show how, in certain situations, things can go wrong.

Example 3.26. $F=1-t^{N}, N>1$ an integer; $B=1$. Clearly (a) is fulfilled, and as

$$
F^{\prime}=-N t^{N-1}, \quad\left(t F^{\prime} / F\right)^{\prime}=-N^{2} t^{N-1} /\left(1-t^{N}\right)^{2},
$$

(b) is likewise fulfilled, at all points $t \in(0, B)$. On the other hand,

$$
F(t)=F(\varepsilon t) \quad \forall t \in \mathbf{D}
$$

whenever $\varepsilon$ is an $N$-th root of unity, and thus (c) is violated. If $\left(\mathrm{d}^{\prime}\right)$ were fulfilled, then Proposition 3.15 would imply that $H^{\infty} \subset A_{\alpha}^{2}$ but $x_{1} \notin A_{\alpha}^{2}$, for all sufficiently large $\alpha$; as $x_{1} \in H^{\infty}$, this is a manifest contradiction, so $\left(\mathrm{d}^{\prime}\right)$ does not hold. Moreover, when we take $x=\left(x_{1}, x_{2}\right)$ and $y=\left(\varepsilon x_{1}, x_{2}\right)$, where $\varepsilon$ is an $N$-th root of unity, then

$$
\frac{a(x, \bar{x}) a(y, \bar{y})}{|a(x, \bar{y})|^{2}}=1 \text {, }
$$

and it even follows that no point of $\mathcal{D}_{F}$ with $x_{1} \neq 0$ is regular. Thus, even if we knew that (d) holds, we would not be able to apply Berezin's procedure $\left(\mathcal{D}_{F}, \omega_{F}\right)$.

Observe that the corresponding domain

$$
\mathcal{D}_{F}=\left\{x \in \mathbf{C}^{2}:\left|x_{2}\right|^{2}+\left|x_{1}\right|^{2 N}<1\right\}
$$


coincides, up to the "flip" $\left(x_{1}, x_{2}\right) \leftrightarrow\left(x_{2}, x_{1}\right)$, with the complex ellipsoid from Example 3.19, with $p=1 / N \in(0,1)$. Thus, while the metric defined on the ellipsoid by the potential

$$
\Psi(x)=-\log \left[\left(1-\left|x_{1}\right|^{2}\right)^{1 / N}-\left|x_{2}\right|^{2}\right]
$$

is quantizable, the pseudometric corresponding to the potential

$$
\Psi(x)=-\log \left[\left(1-\left|x_{2}\right|^{2 N}\right)-\left|x_{1}\right|^{2}\right]
$$

is not.

Example 3.27. A similar situation - i.e. (a) and (b) are fulfilled (the latter for $t \neq 0)$, but no point of $\mathcal{D}_{F}$ is regular - can be shown to prevail for $F=e^{-t^{N}}$ $(B=+\infty)$, or, more generally, for any $F(t)$ which depends only on $t^{N}$ for some integer $N>1$.

The next two examples are concerned with domains $\mathcal{D}_{F}$ for an unbounded function $F$. In this case, the function

$$
A(x \mid y)=\frac{a(x, \bar{x}) a(y, \bar{y})}{|a(x, \bar{y})|^{2}}
$$

is undefined when $x_{1}=0$ or $y_{1}=0$, and, consequently, so is $\psi(x \mid y)=\log A(x \mid y)$. This can easily be remedied as follows. Since we want our function $F$ to be meromorphic on $B \mathbf{D}$, we must have

$$
F(t)=F_{0}(t) / t^{p}
$$

where $p$ is a positive integer and $0<F_{0}(0)<+\infty$. In that case,

$$
A(x \mid y)=\frac{\left[F_{0}\left(\left|x_{1}\right|^{2}\right)-\left|x_{1}^{p} x_{2}\right|^{2}\right] \cdot\left[F_{0}\left(\left|y_{1}\right|^{2}\right)-\left|y_{1}^{p} y_{2}\right|^{2}\right]}{\left|F_{0}\left(x_{1} \bar{y}_{1}\right)-x_{1}^{p} x_{2} \bar{y}_{1}^{p} \bar{y}_{2}\right|^{2}} \quad \text { for } x_{1}, y_{1} \neq 0
$$

But the right-hand side clearly makes sense for $x_{1}, y_{1}=0$ as well, so we can use it to define $A(x \mid y)$ and $\psi(x \mid y)$ on all of $\mathcal{D}_{F} \times \mathcal{D}_{F}$. Then the following analogue of Proposition 3.9 holds.

Proposition 3.28. Let $F(t)=F_{0}(t) / t^{p}$, where $p$ is a positive integer and $F_{0}$ is a function from $B \mathbf{D}$ into $\mathbf{C} \cup\{\infty\}$ which is positive, finite, and $C^{2}$ on $[0, B)$, and let $A(x \mid y)$ be given by (3.45). Then the following are equivalent:

(i) all points $y \in \mathcal{D}_{F}$ with $y_{1} \neq 0$ are regular;

(ii) for all $x, y \in B^{1 / 2} \mathbf{D}$,

$$
F_{0}(x \mid y) \stackrel{\text { def }}{=} \frac{F_{0}\left(|x|^{2}\right) F_{0}\left(|y|^{2}\right)}{\left|F_{0}(x \bar{y})\right|^{2}} \leq 1
$$

and $F_{0}\left(x_{n} \mid y\right) \rightarrow 1$ implies $x_{n} \rightarrow y$;

(iii) $\left|F_{0}(t)\right| \geq F_{0}(|t|) \forall t \in B \mathbf{D}$, with equality iff $t \geq 0$, and $\left(t F_{0}^{\prime} / F_{0}\right)^{\prime}<0$ on $(0, B)$;

(iv) $|F(t)| \geq F(|t|) \forall t \in B \mathbf{D}$, with equality iff $t \geq 0$, and $\left(t F^{\prime} / F\right)^{\prime}<0$ on $(0, B)$. 
The points $x \in \mathcal{D}_{F}$ with $x_{1}=0$ are not regular.

Proof. For $a, b \in \mathcal{D}_{F_{0}}$, denote

$$
A_{0}(a \mid b)=\frac{\left[F_{0}\left(\left|a_{1}\right|^{2}\right)-\left|a_{2}\right|^{2}\right] \cdot\left[F_{0}\left(\left|b_{1}\right|^{2}\right)-\left|b_{2}\right|^{2}\right]}{\left|F_{0}\left(a_{1} \bar{b}_{1}\right)-a_{2} \bar{b}_{2}\right|^{2}},
$$

and for $x=\left(x_{1}, x_{2}\right) \in \mathcal{D}_{F}$ denote by $\hat{x}$ the point $\left(x_{1}, x_{1}^{p} x_{2}\right) \in \mathcal{D}_{F_{0}}$. Thus

$$
A(x \mid y)=A_{0}(\hat{x} \mid \hat{y}) \quad \forall x, y \in \mathcal{D}_{F} .
$$

Now observe that the hypotheses of Proposition 3.9 are satisfied for the function $F_{0}$. It follows that (ii) is equivalent to (iii) and both are, moreover, equivalent to

$$
A_{0}(a \mid b) \leq 1 \quad \forall a, b \in \mathcal{D}_{F_{0}} \text {, and } A_{0}\left(a_{n} \mid b\right) \rightarrow 1 \text { implies } a_{n} \rightarrow b .
$$

Thus, by (3.47), $A(x \mid y) \leq 1$ for all $x, y \in \mathcal{D}_{F}$, and $A\left(x_{n} \mid y\right) \rightarrow 1$ implies $A_{0}\left(\hat{x}_{n} \mid \hat{y}\right) \rightarrow$ 1 implies $\hat{x}_{n} \rightarrow \hat{y}$; if $y_{1} \neq 0$, this further implies that $x_{n} \rightarrow y$. It follows that all points $y \in \mathcal{D}_{F}$ with $y_{1} \neq 0$ are regular. Thus (ii) $\Longleftrightarrow$ (iii) $\Longrightarrow$ (i).

Conversely, assume that (i) holds. Then, in particular, for any $y \in \mathcal{D}_{F}$ with $y_{1} \neq 0$

$$
A(x \mid y) \leq 1 \quad \forall x \in \mathcal{D}_{F}, \text { with equality iff } x=y .
$$

Applying this to $x=\left(x_{1}, 0\right)$ and $y=\left(y_{1}, 0\right)$ shows that, for any $y_{1} \in B^{1 / 2} \mathbf{D}$ with $y_{1} \neq 0$,

$$
F_{0}\left(x_{1} \mid y_{1}\right) \leq 1 \quad \forall x_{1} \in B^{1 / 2} \mathbf{D}, \text { with equality iff } x_{1}=y_{1} .
$$

Now, firstly, take $y_{1}>0$ and $x_{1}=\epsilon y_{1},|\epsilon|=1$; we obtain

$$
\frac{F_{0}\left(\left|y_{1}\right|^{2}\right)^{2}}{\left|F_{0}\left(\epsilon\left|y_{1}\right|^{2}\right)\right|^{2}} \leq 1, \quad \text { with equality iff } \epsilon=1
$$

which is the first part of (iii). Secondly, take $x_{1}=e^{u / 2}, y_{1}=e^{v / 2}, u, v \in$ $(-\infty, \log B)$, and let $\phi_{0} \equiv \phi_{F_{0}}$ be the function associated to $F_{0}$ by (3.2). This gives

$$
F_{0}\left(x_{1} \mid y_{1}\right)=e^{\phi_{0}(u)+\phi_{0}(v)-2 \phi_{0}\left(\frac{u+v}{2}\right)} \leq 1 \quad \text { with equality iff } u=v
$$

In other words, $\phi_{0}$ is strictly concave on $(-\infty, \log B)$, whence $\phi_{0}^{\prime \prime}<0$, or $\left(t F_{0}^{\prime} / F_{0}\right)^{\prime}<$ 0 on $(0, B)$, which is the second part of (iii). Thus, (i) $\Longrightarrow$ (iii).

Since

$$
\frac{t F_{0}^{\prime}}{F_{0}}=\frac{t F^{\prime}}{F}-p
$$

the equivalence (iii) $\Longleftrightarrow$ (iv) is immediate.

Finally, for $x, y \in \mathcal{D}_{F}$ with $x_{1}=y_{1}=0$, we have by (3.45)

$$
A(x \mid y)=\frac{F_{0}(0) \cdot F_{0}(0)}{\left|F_{0}(0)\right|^{2}}=1 \quad \text { for all } x_{2} \text { and } y_{2} .
$$

Consequently, no point $y \in \mathcal{D}_{F}$ with $y_{1}=0$ can be regular. 
Example 3.29. $F(t)=1 / t e^{t} ; B=+\infty$. Then $F$ is meromorphic in $\mathbf{C}$, with the only pole at $t=0$. For $t>0$, we have $F(t)>0$,

$$
F^{\prime}(t)=-\left(\frac{1}{t^{2}}+\frac{1}{t}\right) e^{-t}<0, \quad\left(\frac{t F^{\prime}}{F}\right)^{\prime}=(-1-t)^{\prime}=-1<0,
$$

so (b) is fulfilled. Further,

$$
c_{k}\left(F^{\alpha}\right)=\int_{0}^{+\infty} t^{k-\alpha} e^{-\alpha t} d t= \begin{cases}+\infty & \text { if } k<\alpha \\ (k-\alpha) ! \alpha^{\alpha-k-1} & \text { if } k \geq \alpha\end{cases}
$$

Consequently,

$$
\sum_{k=0}^{\infty} t^{k} / c_{k}\left(F^{\alpha}\right)=\sum_{k=\alpha}^{\infty} \frac{t^{k} \alpha^{k-\alpha+1}}{(k-\alpha) !}=\alpha t^{\alpha} e^{\alpha t}=\alpha F(t)^{-\alpha},
$$

and we see that $\left(d^{\prime}\right)$ is satisfied with $\gamma=1$. The reproducing kernels are given by

$$
K_{\alpha}(x, \bar{y})=(\alpha-2)\left[\alpha-x_{2} \bar{y}_{2} x_{1} \bar{y}_{1} e^{x_{1} \bar{y}_{1}}\right] \cdot\left[e^{x_{1} \bar{y}_{1}} /\left(x_{1} \bar{y}_{1}\right)-x_{2} \bar{y}_{2}\right]^{-\alpha} .
$$

However, by Proposition $3.28, \mathcal{D}_{F}$ contains points which are not regular. Hence, though the reproducing kernels have the required asymptotic behaviour, Berezin's quantization cannot be done.

This situation admits an interpretation similar to that in Example 3.26. Namely, write $F(t)=F_{0}(t) / t$, where $F_{0}(t)=e^{-t}$. The mapping

$$
x=\left(x_{1}, x_{2}\right) \in \mathcal{D}_{F} \mapsto \hat{x}=\left(x_{1}, x_{1} x_{2}\right) \in \mathcal{D}_{F_{0}}
$$

is a biholomorphism of $\widetilde{\mathcal{D}}=\left\{x \in \mathcal{D}_{F}, x_{1} \neq 0\right\}$ onto $\widetilde{\mathcal{D}}_{0}=\left\{x \in \mathcal{D}_{F_{0}}, x_{1} \neq 0\right\}$. Moreover, the sets $\mathcal{D}_{F} \backslash \widetilde{\mathcal{D}}$ and $\mathcal{D}_{F_{0}} \backslash \widetilde{\mathcal{D}}_{0}$ are $A_{\alpha}^{2}$-negligible - that is, the restriction $f \mapsto f \mid \widetilde{\mathcal{D}}$ is an isometric isomorphism of $A^{2}\left(\mathcal{D}_{F}, a_{F}^{\alpha} d \mu\right)$ onto $A^{2}\left(\widetilde{\mathcal{D}}, a_{F}^{\alpha} d \mu\right)$, and similarly for $\mathcal{D}_{F_{0}}$ and $\widetilde{\mathcal{D}}_{0}$. It can easily be shown that this implies that the quantization of $\mathcal{D}_{F}$ with the Kähler potential

$$
\Psi=-\log a_{F}=-\log \left[1 /\left(\left|x_{1}\right|^{2} e^{\left|x_{1}\right|^{2}}\right)-\left|x_{2}\right|^{2}\right]
$$

is equivalent to the quantization of $\mathcal{D}_{F_{0}}$ with the Kähler potential

$$
\Psi=-\log \left[e^{-\left|x_{1}\right|^{2}}-\left|x_{1} x_{2}\right|^{2}\right]
$$

(We omit the details. ${ }^{9}$ ) But $\mathcal{D}_{F_{0}}$ is exactly the Springer domain from Example 3.20. Thus, the metric on the Springer domain corresponding to the potential

$$
\Psi=-\log \left[e^{-\left|x_{1}\right|^{2}}-\left|x_{2}\right|^{2}\right]
$$

is quantizable, whereas the one corresponding to the potential (3.48) is not. A similar comment can be made about the following example, which even exhibits (for $p=1$ ) an unquantizable singular Kähler metric on the unit ball $\mathbf{B}_{2}$ in $\mathbf{C}^{2}$.

\footnotetext{
${ }^{9}$ See Remark 5.7.
} 
Example 3.30. $F(t)=(1-t)^{p} / t, p>0 ; B=1$. Again, $F$ is analytic on $\mathbf{D}$ except for a simple pole at $t=0$. For $t>0, F(t)>0$,

$$
F^{\prime}(t)=-\frac{(1-t)^{p}}{t^{2}}-p \frac{(1-t)^{p-1}}{t}<0, \quad\left(\frac{t F^{\prime}}{F}\right)^{\prime}=\frac{-p}{(1-t)^{2}}<0,
$$

so (b) is satisfied. Further,

$$
c_{k}\left(F^{\alpha}\right)=p \int_{0}^{1} t^{k-\alpha}(1-t)^{p \alpha-2} d t=\frac{p(k-\alpha) !(p \alpha-2) !}{(k-\alpha+p \alpha-1) !} \quad \text { for } k \geq \alpha,
$$

and equals $+\infty$ for $k<\alpha$. Consequently,

$\sum_{k=0}^{\infty} t^{k} / c_{k}\left(F^{\alpha}\right)=\frac{1}{p} \sum_{j=0}^{\infty} t^{j+\alpha} \frac{(j+p \alpha-1) !}{j !(p \alpha-2) !}=\frac{p \alpha-1}{p} t^{\alpha}(1-t)^{-p \alpha}=\left(\alpha-\frac{1}{p}\right) F(t)^{-\alpha}$,

and $\left(\mathrm{d}^{\prime}\right)$ is fulfilled with $\gamma=1-\frac{1}{p}$. However, the points $x \in \mathcal{D}_{F}$ with $x_{1} \neq 0$ are not regular, by Proposition 3.28. Hence, again, $\left(\mathcal{D}_{F}, \omega_{F}\right)$ is not quantizable, even though the reproducing kernels

$$
\begin{gathered}
K_{\alpha}(x, \bar{y})=(\alpha-2)\left[(\alpha-1)+\left(1-\frac{1}{p}\right)\left(1-x_{2} \bar{y}_{2} x_{1} \bar{y}_{1}\left(1-x_{1} \bar{y}_{1}\right)^{-p}\right)\right] \\
\cdot\left[\left(1-x_{1} \bar{y}_{1}\right)^{p} /\left(x_{1} \bar{y}_{1}\right)-x_{2} \bar{y}_{2}\right]^{-\alpha}
\end{gathered}
$$

have the required asymptotic behaviour.

For $p=1,(3.49)$ reduces to

$$
K_{\alpha}(x, \bar{y})=(\alpha-2)(\alpha-1) \cdot a(x, \bar{y})^{-\alpha}
$$

for all integers $\alpha \geq 3$. This is Berezin's assumption (A). Apart from $\Omega=\mathbf{C}^{N}$ and $\Omega$ a bounded symmetric domain, this is the only situation we know where the assumption (A) is satisfied. (It must, however, be emphasized that this metric has cusps at all points with $z_{1}=0$.)

Example 3.31. $F=(1-\sqrt{t})^{2}, B=1$. This last example, announced already in Section 2, exhibits a singular Kähler manifold $\left(\mathcal{D}_{F}, \omega_{F}\right)$ for which $\left(\mathrm{A}^{\prime}\right)$ holds for all $x=y$ with $x_{1} \neq 0$, but fails for $x=y$ with $x_{1}=0$. Note that the domain $\mathcal{D}_{F}$ can be written as

$$
\mathcal{D}_{F}=\left\{z \in \mathbf{C}^{2}:\left|z_{1}\right|+\left|z_{2}\right|<1\right\}
$$

i.e. $\mathcal{D}_{F}$ is a "complex cube".

Since $F$ is continuous and decreasing on $[0,1)$ and

$$
\left(\frac{t F^{\prime}}{F}\right)^{\prime}=-\frac{1}{2 \sqrt{t}(1-\sqrt{t})^{2}}<0
$$

on $(0,1), \mathcal{D}_{F}$ is a pseudoconvex Reinhardt domain by Corollary 3.2, and Proposition 3.12 can be applied. We have

$$
c_{k}\left(F^{\alpha}\right)=\int_{0}^{1} t^{k}(1-\sqrt{t})^{2 \alpha-2} \frac{d t}{2 \sqrt{t}}=\int_{0}^{1} s^{2 k}(1-s)^{2 \alpha-2} d s=\frac{(2 k) !(2 \alpha-2) !}{(2 k+2 \alpha-1) !} .
$$


Consequently,

$$
\begin{aligned}
\sum_{k=0}^{\infty} t^{k} / c_{k}\left(F^{\alpha}\right) & =(2 \alpha-1) \sum_{k=0}^{\infty}\left(\begin{array}{c}
2 k+2 \alpha-1 \\
2 k
\end{array}\right) t^{k} \\
& =(2 \alpha-1) \sum_{j=0}^{\infty}\left(\begin{array}{c}
j+2 \alpha-1 \\
j
\end{array}\right) \frac{\sqrt{t}^{j}+(-\sqrt{t})^{j}}{2} \\
& =\frac{2 \alpha-1}{2}\left[(1-\sqrt{t})^{-2 \alpha}+(1+\sqrt{t})^{-2 \alpha}\right]
\end{aligned}
$$

Substituting this into (3.35) and setting, for brevity, $t_{i}=x_{i} \bar{y}_{i}$, we obtain

$$
\begin{aligned}
& K_{\alpha}(x, \bar{y})=(\alpha-2) \sum_{l=0}^{\infty}\left(\begin{array}{c}
l+\alpha-2 \\
l
\end{array}\right) t_{2}^{l}\left(l+\alpha-\frac{1}{2}\right) \sum_{\epsilon= \pm 1}\left(1-\epsilon \sqrt{t_{1}}\right)^{-2 l-2 \alpha} \\
&=(\alpha-2) \sum_{\epsilon= \pm 1}\left(1-\epsilon \sqrt{t_{1}}\right)^{-2 \alpha} \\
& \cdot \sum_{l=0}^{\infty}\left[(\alpha-1)\left(\begin{array}{c}
l+\alpha-1 \\
l
\end{array}\right)+\frac{1}{2}\left(\begin{array}{c}
l+\alpha-2 \\
l
\end{array}\right)\right] \frac{t_{2}^{l}}{\left(1-\epsilon \sqrt{t_{1}}\right)^{2 l}} \\
&=(\alpha-2) \sum_{\epsilon= \pm 1}\left(1-\epsilon \sqrt{t_{1}}\right)^{-2 \alpha} \\
& \cdot\left[(\alpha-1)\left(1-\frac{t_{2}}{\left(1-\epsilon \sqrt{t_{1}}\right)^{2}}\right)^{-\alpha}+\frac{1}{2}\left(1-\frac{t_{2}}{\left(1-\epsilon \sqrt{t_{1}}\right)^{2}}\right)^{1-\alpha}\right] \\
&=(\alpha-2) \sum_{\epsilon= \pm 1}\left[(\alpha-1)+\frac{1}{2}\left(1-\frac{t_{2}}{\left(1-\epsilon \sqrt{t_{1}}\right)^{2}}\right)\right] \cdot\left[\left(1-\epsilon \sqrt{t_{1}}\right)^{2}-t_{2}\right]^{-\alpha}
\end{aligned}
$$

When $x=y$ and $x_{1} \neq 0$, then $t_{1}>0$, so $0<\left(1-\sqrt{t_{1}}\right)^{2}-t_{2}<\left(1+\sqrt{t_{1}}\right)^{2}-t_{2}$, and $K_{\alpha}(x, \bar{x}) \cdot\left[\left(1-\left|x_{1}\right|\right)^{2}-\left|x_{2}\right|^{2}\right]^{\alpha}=(\alpha-2)(\alpha-1)+(\alpha-2) \cdot \frac{1}{2}\left(1-\frac{\left|x_{2}\right|^{2}}{\left(1-\left|x_{1}\right|\right)^{2}}\right)+o(1)$ as $\alpha \rightarrow+\infty$. Therefore (d), or (2.17), is fulfilled in this case. On the other hand, for $x_{1}=0$ we have

$$
K_{\alpha}(x, \bar{x}) \cdot\left[1-\left|x_{2}\right|^{2}\right]^{\alpha}=2(\alpha-2)(\alpha-1)+(\alpha-2)\left(1-\left|x_{2}\right|^{2}\right) .
$$

Thus, the function

$$
\lim _{\alpha \rightarrow+\infty} \frac{K_{\alpha}(x, \bar{x})\left[\left(1-\left|x_{1}\right|\right)^{2}-\left|x_{2}\right|^{2}\right]^{\alpha}}{\alpha^{2}}
$$

has a jump discontinuity - it equals one for $x_{1} \neq 0$ and 2 for $x_{1}=0$.

A similar situation can be shown to prevail on the unit disc $\mathbf{D}$ with the Kähler metric given by the potential $\log (1-|z|)$. In both cases, however, the metric has singularities. The author does not know of any similar example with a non-singular Kähler manifold. 
Problem. Given a Kähler manifold $(\Omega, \omega)$ of dimension $n$ with potential $\Psi$ and $a=e^{-\Psi}$, is it always true that the limit

$$
\lim _{\alpha \in \mathbf{N}, \alpha \rightarrow+\infty}\left(K_{\alpha}(x, \bar{x})\right)^{-1 / \alpha}:=\rho(x)
$$

exists and

$$
\lim _{\alpha \in \mathbf{N}, \alpha \rightarrow+\infty} \alpha^{-n} K_{\alpha}(x, \bar{x}) \rho(x)^{\alpha}=1
$$

for each $x \in \Omega$ ?

In the special case of $\Omega=\mathbf{D}$ and a rotation-invariant $\omega$, this problem has already been posed in [E2].

\section{An alternative approach}

On some Kähler manifolds $\Omega$ there may exist other "natural" measures apart from the Liouville measure $d \mu$. For instance, this is certainly the case when $\Omega$ is a domain in $\mathbf{C}^{n}$, where it is very straightforward to work with the Lebesgue measure $d z$ and try to construct the quantization using the chain of measures $a(z)^{\alpha} d z$ instead of $a(z)^{\alpha} d \mu(z)$. This will be done in the present section. As the exposition runs almost parallel to that in Section 2, we will proceed at a more rapid pace and skip some of the details.

Thus, let $\Omega$ be a domain in $\mathbf{C}^{n}, \omega=\sum g_{j \bar{k}} d z_{j} \wedge d \bar{z}_{k}$ a Kähler form on $\Omega, \Psi$ a (real-valued) potential for $\omega$, and $g(z)=\operatorname{det}\left(g_{j \bar{k}}(z)\right)$. For $\alpha \geq 0$, let $\widetilde{A}_{\alpha}^{2}$ be the Bergman space of all holomorphic functions on $\Omega$ which are square integrable with respect to the measure

$$
e^{-\alpha \Psi(x)} d x / \pi^{n},
$$

where $d x$ is the (2n-dimensional) Lebesgue measure in $\mathbf{C}^{n}$. It may well happen ${ }^{10}$ that $\widetilde{A}_{\alpha}^{2}=\{0\}$; in any case, however, it follows from the continuity and positivity of $e^{-\alpha \Psi}$ and from the mean value property of holomorphic functions that the evaluation functionals at all $x \in \Omega$ are continuous on $\widetilde{A}_{\alpha}^{2}$, and, consequently, $\widetilde{A}_{\alpha}^{2}$ possesses a reproducing kernel $\widetilde{K}_{\alpha}(x, \bar{y})$. The Berezin transform with respect to $\widetilde{A}_{\alpha}^{2}$ is defined as

$$
\widetilde{B}_{\alpha} f(y)=\int_{\Omega} f(x) \frac{\left|\widetilde{K}_{\alpha}(x, \bar{y})\right|^{2}}{\widetilde{K}_{\alpha}(y, \bar{y})} a(x, \bar{x})^{\alpha} \frac{d x}{\pi^{n}} .
$$

We make the following assumptions about the objects we have just introduced.

Assumption ( $\left.\mathrm{E}^{*}\right)$. The function

$$
a(x, \bar{x}):=e^{-\Psi(x)}
$$

extends to a sesquimeromorphic function $a(x, \bar{y})$ on $\Omega \times \Omega$.

Since

$$
g_{j \bar{k}}(z)=\frac{\partial^{2}}{\partial z_{j} \partial \bar{z}_{k}}[-\log a(z, \bar{z})]=\frac{1}{a^{2}} \frac{\partial a}{\partial z_{j}} \frac{\partial a}{\partial \bar{z}_{k}}-\frac{1}{a} \frac{\partial^{2} a}{\partial z_{j} \partial \bar{z}_{k}},
$$

${ }^{10}$ We remark, however, that when $\Omega$ is bounded and pseudoconvex, $\widetilde{A}_{\alpha}^{2}$ is nontrivial for all $\alpha \geq 0$; this is a theorem due to Bombieri (see [M], Proposition 1.3). 
it follows that the metric coefficients $g_{j \bar{k}}$ also extend to sesquimeromorphic functions $g_{j \bar{k}}(x, \bar{y})$ on $\Omega \times \Omega$, and, consequently, so does their determinant:

$$
g(x, \bar{y}):=\operatorname{det}\left(g_{j \bar{k}}(x, \bar{y})\right) .
$$

Introduce the (real-valued) function

$$
\gamma(x \mid y):=\frac{|g(x, \bar{y})|^{2}}{g(x, \bar{x}) g(y, \bar{y})}, \quad x, y \in \Omega
$$

and also keep the previous notation

$$
\psi(x \mid y)=\log \frac{a(x, \bar{x}) a(y, \bar{y})}{|a(x, \bar{y})|^{2}} .
$$

Note that $\gamma(x \mid y) \geq 0$ for all $x$ and $y$. Recall that a point $y \in \Omega$ was called regular if $\psi(\cdot \mid y) \leq 0$ and $\psi\left(x_{n} \mid y\right) \rightarrow 0$ implied $x_{n} \rightarrow y$.

Assumption ( $\left.\mathrm{C}^{*} 1\right)$. All points of $\Omega$ are regular.

Assumption $\left(\mathrm{C}^{*} 2\right) \cdot \gamma(x \mid y) \leq 1$ for all $x, y \in \Omega$.

Assumption $\left(\mathrm{A}^{*}\right)$. There exists an infinite subset $E$ of the positive integers such that for all $\alpha \in E$,

$$
\widetilde{K}_{\alpha}(x, \bar{y}) a(x, \bar{y})^{\alpha} / g(x, \bar{y})=c_{n} \alpha^{n}+B(x, \bar{y}) \alpha^{n-1}+C(x, \bar{y}, \alpha)
$$

where $c_{n} \in \mathbf{C}$ and $B, C$ are bounded functions, sesquianalytic in $(x, \bar{y})$, on $\Omega \times \Omega$ and $\Omega \times \Omega \times E$, respectively.

For $\alpha \in E$ and $h=1 / \alpha$, denote by $\mathcal{A}_{h}$ the algebra of covariant symbols for $\widetilde{A}_{\alpha}^{2}$, and construct the algebra $\mathcal{A}$ and its linear subset $\widetilde{\mathcal{A}}$ in the same way as in Section 2 . Then we have the following analogues of Theorems 2.5, 2.10, 2.11, 2.12 and 2.12A.

Theorem 4.1. Suppose that $\left(\mathrm{E}^{*}\right)$ and $\left(\mathrm{A}^{*}\right)$ are fulfilled, $y \in \Omega$ is regular and $\gamma(\cdot \mid y) \leq 1$. Let $f$ be any function on $\Omega$ which is $C^{3}$ in a neighbourhood of $y$ and for which the integral (4.2) exists for some $\alpha=\alpha_{1}$ (for instance, $f$ can be bounded and measurable). Then the Berezin transform (4.2) exists for all $\alpha \geq \alpha_{1}$ and satisfies

$$
\widetilde{B}_{\alpha} f(y)=f(y)+\alpha^{-1} \Delta f(y)+o\left(\alpha^{-1}\right)
$$

as $\alpha \rightarrow+\infty, \alpha \in E$, where $\Delta$ is the Laplace-Beltrami operator on $\Omega$ (given by $(2.23))$.

Proof. In view of $\left(\mathrm{A}^{*}\right)$, we have

$$
\frac{\left|\widetilde{K}_{\alpha}(x, \bar{y})\right|^{2}}{\widetilde{K}_{\alpha}(y, \bar{y})}=\frac{a(y, \bar{y})^{\alpha}}{|a(x, \bar{y})|^{2 \alpha}} \cdot \frac{|g(x, \bar{y})|^{2}}{g(y, \bar{y})} \cdot c_{n} \alpha^{n}\left[1+\frac{\widetilde{B}(x, y)}{\alpha}+\frac{\widetilde{C}(x, y, \alpha)}{\alpha^{2}}\right],
$$

where $\widetilde{B}(x, y)=c_{n}^{-1}[B(x, \bar{y})+B(y, \bar{x})-B(y, \bar{y})]$ and $\widetilde{C}(x, y, \alpha)$ are bounded functions on $\Omega \times \Omega$ and $\Omega \times \Omega \times E$, respectively. Therefore

(4.7) $\frac{\left|\widetilde{K}_{\alpha}(x, \bar{y})\right|^{2}}{\widetilde{K}_{\alpha}(y, \bar{y})} \cdot a(x, \bar{x})^{\alpha} \frac{d x}{\pi^{n}}$

$$
=e^{\alpha \psi(x \mid y)} \cdot c_{n} \alpha^{n}\left[1+\frac{\widetilde{B}(x, y)}{\alpha}+\frac{\widetilde{C}(x, y, \alpha)}{\alpha^{2}}\right] \cdot \gamma(x \mid y) d \mu(x)
$$


and

$$
\begin{aligned}
\widetilde{B}_{\alpha} f(y)= & c_{n} \alpha^{n} \int_{\Omega} f(x) \gamma(x \mid y) e^{\alpha \psi(x \mid y)} d \mu(x) \\
& +c_{n} \alpha^{n-1} \int_{\Omega} f(x) \widetilde{B}(x, y) \gamma(x \mid y) e^{\alpha \psi(x \mid y)} d \mu(x) \\
& +c_{n} \alpha^{n-2} \int_{\Omega} f(x) \widetilde{C}(x, y, \alpha) \gamma(x \mid y) e^{\alpha \psi(x \mid y)} d \mu(x) .
\end{aligned}
$$

The absolute value of the last summand on the RHS does not exceed

$$
\sup |\widetilde{C}(x, y, \alpha)| \cdot c_{n} \alpha^{n-2} \int_{\Omega}|f(x)| e^{\alpha \psi(x \mid y)} d \mu(x) .
$$

It follows from the hypotheses and from the boundedness of $\widetilde{B}$ and $\gamma$ that Lemma 2.2 can be applied to the first two summands in (4.8) and to the integral in (4.9). As $\gamma(y \mid y)=1$, this yields

$$
c_{n}^{-1} \widetilde{B}_{\alpha}(y)=f(y)+\frac{\Delta(\gamma(\cdot \mid y) f)(y)+\sigma(y) f(y)}{\alpha}+\frac{f(y) c_{n}^{-1} B(y, \bar{y})}{\alpha}+o\left(\alpha^{-1}\right)
$$

as $\alpha \rightarrow+\infty$. A lengthy but straightforward computation shows that

$$
\begin{aligned}
\left.\Delta_{x}[\gamma(x \mid y) f(x)]\right|_{x=y} & =\Delta f(y)+f(y) \cdot \Delta \ln g(y, \bar{y}) \\
& =\Delta f(y)-\frac{2}{3} \sigma(y) f(y)
\end{aligned}
$$

whence

$$
c_{n}^{-1} \widetilde{B}_{\alpha} f(y)=f(y)+\frac{\Delta f(y)+\left[\frac{1}{3} \sigma(y)+c_{n}^{-1} B(y, \bar{y})\right] f(y)}{\alpha}+o\left(\alpha^{-1}\right) .
$$

As $\widetilde{B}_{\alpha} \mathbf{1}=\mathbf{1}$, we have, in particular,

$$
c_{n}^{-1}=1+\left[\frac{1}{3} \sigma(y)+c_{n}^{-1} B(y, \bar{y})\right] \alpha^{-1}+o\left(\alpha^{-1}\right) .
$$

Therefore $c_{n}=1$,

$$
B(y, \bar{y})+\frac{1}{3} \sigma(y)=0,
$$

and the assertion of the theorem follows.

The formula (4.10) deserves to be stated separately.

Corollary 4.2. Assume that $\left(\mathrm{E}^{*}\right)$ and $\left(\mathrm{A}^{*}\right)$ are fulfilled and that $y$ is a regular point for which $\gamma(\cdot \mid y) \leq 1$. Then $c_{n}=1$ and

$$
B(y, \bar{y})=-\frac{1}{3} \Delta \ln g(y, \bar{y})
$$

In particular, if $\left(\mathrm{C}^{*} 1\right)$ and $\left(\mathrm{C}^{*} 2\right)$ also hold, then

$$
B(x, \bar{y})=-\frac{1}{3} \Delta \ln g(x, \bar{y})
$$

for all $x, y \in \Omega$. 
Theorem 4.3. Assume that $\left(\mathrm{E}^{*}\right),\left(\mathrm{A}^{*}\right),\left(\mathrm{C}^{*} 1\right)$ and $\left(\mathrm{C}^{*} 2\right)$ are fulfilled and let $f$, $g \in \widetilde{\mathcal{A}}$. Then

$$
(f * g)(h \mid z)=f(0 \mid z) g(0 \mid z)+o(1),
$$

and

$$
\frac{1}{h}(f * g-g * f)(h \mid z)=\frac{1}{i}\{f, g\}(0 \mid z)+o(1)
$$

as $\alpha=1 / h \rightarrow+\infty, \alpha \in E$, where $\{\cdot, \cdot\}$ is the Poisson bracket on $\Omega$.

Proof. Start as in the proof of Theorem 2.10, defining the functions $u, u_{1}$ and $u_{2}$ and writing down the analogue of the formula (2.37):

$$
\begin{aligned}
(f * g)(h \mid z)= & \int_{\Omega} u(0 \mid x, \bar{x}) \frac{\left|\widetilde{K}_{\alpha}(x, \bar{z})\right|^{2}}{\widetilde{K}_{\alpha}(z, \bar{z})} a(x, \bar{x})^{\alpha} \frac{d x}{\pi^{n}} \\
& +h \int_{\Omega} u_{1}(x, \bar{x}) \frac{\left|\widetilde{K}_{\alpha}(x, \bar{z})\right|^{2}}{\widetilde{K}_{\alpha}(z, \bar{z})} a(x, \bar{x})^{\alpha} \frac{d x}{\pi^{n}} \\
& +h^{2} \int_{\Omega} u_{2}(h \mid x, \bar{x}) \frac{\left|\widetilde{K}_{\alpha}(x, \bar{z})\right|^{2}}{\widetilde{K}_{\alpha}(z, \bar{z})} a(x, \bar{x})^{\alpha} \frac{d x}{\pi^{n}} .
\end{aligned}
$$

By $(\widetilde{\mathcal{A}} 2)$ again, the function $u_{2}$ satisfies

$$
h^{-n} \int_{\Omega}\left|u_{2}(h \mid x, \bar{x})\right| e^{\alpha \psi(x \mid z)} d \mu(x) \leq r(z)<+\infty \quad \forall h \in\left(0, h_{0}\right) .
$$

In view of the formula (4.7) and $\left(\mathrm{C}^{*} 2\right)$, this implies

$$
\int_{\Omega}\left|u_{2}(h \mid x, \bar{x})\right| \frac{\left|\widetilde{K}_{\alpha}(x, \bar{z})\right|^{2}}{\widetilde{K}_{\alpha}(z, \bar{z})} a(x, \bar{x})^{\alpha} \frac{d x}{\pi^{n}} \leq \widetilde{r}(z)<+\infty \quad \forall h \in\left(0, h_{0}\right),
$$

with $\widetilde{r}(z)$ independent of $h$. It follows that the third summand on the RHS of (4.13) is $O\left(h^{2}\right)$. A similar argument shows that it is legitimate to apply Theorem 4.1 to the first two summands in (4.13), and we obtain

$$
(f * g)(h \mid z)=u(0 \mid z, \bar{z})+h \cdot\left[\left.\Delta_{x} u(0 \mid x, \bar{x})\right|_{x=z}+u_{1}(z, \bar{z})\right]+o(h) .
$$

From here, (4.11) and (4.12) follow in the same way as in the proof of Theorem 2.10 .

Theorem 4.4. Assume that $\left(\mathrm{E}^{*}\right),\left(\mathrm{A}^{*}\right),\left(\mathrm{C}^{*} 1\right)$ and $\left(\mathrm{C}^{*} 2\right)$ are satisfied. Then for any two distinct points $z_{0}, z_{1} \in \Omega$ there exists a function $f \in \widetilde{\mathcal{A}}$ such that $f\left(0 \mid z_{0}\right) \neq f\left(0 \mid z_{1}\right)$.

Proof. We shall temporarily use $\|\cdot\|_{\alpha}$ and $\|\cdot\|_{\tilde{\alpha}}$ to denote the norms in $A_{\alpha}^{2}$ and $\widetilde{A}_{\alpha}^{2}$, respectively. As in the proof of Theorem 2.11, consider the function (denoted there by $g$ )

$$
h(z)=\left[\sqrt{a\left(z_{0}, \bar{z}_{0}\right)} / a\left(z, \bar{z}_{0}\right)\right]^{\alpha_{1}}
$$


and remember that $h$ was shown to belong to $A_{\alpha_{1}}^{2}$ when $\alpha_{1}$ is sufficiently large. Fix such an $\alpha_{1}$, and, as before, define the functions $\epsilon(h)$ and

$$
f(h \mid x)=\epsilon(h) h(x) \overline{h(x)} a(x, \bar{x})^{\alpha_{1}}=\epsilon(h) e^{\alpha_{1} \psi\left(x \mid z_{0}\right)} .
$$

We shall show that, once again, this function does the job we want. It has been checked in the proof of Theorem 2.11 that $f(0 \mid x)$ separates $z_{0}$ from $z_{1}$ and that $f$ satisfies the conditions $(\widetilde{\mathcal{A}} 1)$ and $(\widetilde{\mathcal{A}} 2)$. Thus it only remains to prove that $f(h \mid \cdot)$ is a covariant symbol of some bounded linear operator on $\widetilde{A}_{\alpha}^{2}(\alpha=1 / h)$, i.e. that the operator

$$
A_{h} F(x):=\int_{\Omega} f(h \mid x, \bar{y}) \widetilde{K}_{\alpha}(x, \bar{y}) \cdot F(y) a(y, \bar{y})^{\alpha} \frac{d y}{\pi^{n}}
$$

is bounded on $\widetilde{A}_{\alpha}^{2}$. By the Schwarz inequality,

$$
\begin{aligned}
\left|A_{h} F(x)\right|^{2} & =\left|\epsilon(h) h(x) \int_{\Omega} \overline{h(y)} a(x, \bar{y})^{\alpha_{1}} \widetilde{K}_{\alpha}(x, \bar{y}) F(y) a(y, \bar{y})^{\alpha} \frac{d y}{\pi^{n}}\right|^{2} \\
& \leq \epsilon(h)^{2} h(x)^{2}\|F\|_{\widetilde{\alpha}}^{2} \cdot \int_{\Omega}|h(y)|^{2} \cdot\left|\widetilde{K}_{\alpha}(x, \bar{y}) a(x, \bar{y})^{\alpha_{1}}\right|^{2} \cdot a(y, \bar{y})^{\alpha} \frac{d y}{\pi^{n}} .
\end{aligned}
$$

Owing to $\left(\mathrm{A}^{*}\right)$, we may suppose that $\alpha_{1}$ has been chosen so large that

$$
\left|\widetilde{K}_{\alpha}(x, \bar{y})\right| \leq 2 \alpha^{n}|a(x, \bar{y})|^{-\alpha} \cdot|g(x, \bar{y})| \quad \forall \alpha>\alpha_{1}, \forall x, y \in \Omega .
$$

In view of $\left(\mathrm{C}^{*} 1\right)$ and $\left(\mathrm{C}^{*} 2\right)$, we then have

$$
\begin{aligned}
\left|\widetilde{K}_{\alpha}(x, \bar{y}) a(x, \bar{y})^{\alpha_{1}}\right|^{2} & \leq 4 \alpha^{2 n}|a(x, \bar{y})|^{-2\left(\alpha-\alpha_{1}\right)} \cdot|g(x, \bar{y})|^{2} \\
& \leq 4 \alpha^{2 n} a(x, \bar{x})^{\alpha_{1}-\alpha} a(y, \bar{y})^{\alpha_{1}-\alpha} g(x, \bar{x}) g(y, \bar{y}) .
\end{aligned}
$$

The last integral therefore does not exceed

$$
\begin{gathered}
4 \alpha^{2 n} a(x, \bar{x})^{\alpha_{1}-\alpha} g(x, \bar{x}) \int_{\Omega}|h(y)|^{2} a(y, \bar{y})^{\alpha_{1}} g(y, \bar{y}) \frac{d y}{\pi^{n}} \\
=4 \alpha^{2 n} a(x, \bar{x})^{\alpha_{1}-\alpha} g(x, \bar{x})\|h\|_{\alpha_{1}}^{2}
\end{gathered}
$$

(because $\left.g(y, \bar{y}) d y / \pi^{n}=d \mu(y) !\right)$, and

$$
\begin{aligned}
\left\|A_{h} F\right\|_{\widetilde{\alpha}}^{2} & \leq \epsilon(h)^{2}\|F\|_{\tilde{\alpha}}^{2} \cdot 4 \alpha^{2 n}\|h\|_{\alpha_{1}}^{2} \int_{\Omega}|h(x)|^{2} a(x, \bar{x})^{\alpha_{1}-\alpha} g(x, \bar{x}) \cdot a(x, \bar{x})^{\alpha} \frac{d x}{\pi^{n}} \\
& =\epsilon(h)^{2} \cdot\|F\|_{\widetilde{\alpha}}^{2} \cdot 4 \alpha^{2 n}\|h\|_{\alpha_{1}}^{4} .
\end{aligned}
$$

Thus $\left\|A_{h}\right\| \leq 2 \alpha^{n} \epsilon(h)\|h\|_{\alpha_{1}}^{2}<+\infty$, and the proof is finished.

Theorem 4.5. Assume that $\left(\mathrm{E}^{*}\right),\left(\mathrm{A}^{*}\right),\left(\mathrm{C}^{*} 1\right)$ and $\left(\mathrm{C}^{*} 2\right)$ are fulfilled. Then the algebra $\mathcal{A}$ is a quantization of $(\Omega, \omega)$ satisfying the weak form of the correspondence principle.

Proof. Corollary to the preceding two theorems.

For $\Omega$ a bounded domain, we have also the following analogues of Theorems $2.11 \mathrm{~A}$ and $2.12 \mathrm{~A}$. Their proofs are completely identical to those of $2.11 \mathrm{~A}$ and $2.12 \mathrm{~A}$, so we will not repeat them here. 
Theorem 4.4A. Assume that $\Omega$ is bounded and $\left(\mathrm{E}^{*}\right)$ holds, and that $\left(\mathrm{A}^{*}\right)$ holds but with $B(x, \bar{y})$ and $C(x, \bar{y}, \alpha)$ merely bounded for each fixed $y \in \Omega$. Then for any two distinct points $z_{0}, z_{1} \in \Omega$ there exists $f \in \widetilde{\mathcal{A}}$ such that $f\left(0 \mid z_{0}\right) \neq f\left(0 \mid z_{1}\right)$.

Theorem 4.5A. Assume that $\Omega$ is bounded and $\left(\mathrm{E}^{*}\right),\left(\mathrm{C}^{*} 1\right)$ and $\left(\mathrm{C}^{*} 2\right)$ hold, and that $\left(\mathrm{A}^{*}\right)$ holds but with $B(x, \bar{y})$ and $C(x, \bar{y}, \alpha)$ merely bounded for each fixed $y \in \Omega$. Then the algebra $\mathcal{A}$ is a quantization of $(\Omega, \omega)$ satisfying the weak form of the correspondence principle.

Let us test our result on the domains from Examples 2.13-2.16.

Example 4.6. $\Omega=\mathbf{C}^{n}, \Psi(z)=|z|^{2}$. Then $g=\operatorname{det} \delta_{j k}=1$, and $d \mu(z)$ coincides with the normalized Lebesgue measure $d z / \pi^{n}$. Thus $A_{\alpha}^{2}=\widetilde{A}_{\alpha}^{2}, K_{\alpha}=\widetilde{K}_{\alpha}$ and $B_{\alpha}=\widetilde{B}_{\alpha}$, i.e. both quantization procedures coincide.

Example 4.7. $\Omega$ is a bounded symmetric domain in $\mathbf{C}^{n}$, with the Kähler form given by the potential $\Psi(x)=\log K(x, \bar{x})$, where $K(x, \bar{y})$ is the Bergman kernel function as in Example 2.14; in our present notation, $K$ is the same thing as $\widetilde{K}_{0}$. It is well known (cf. e.g. [Ber1], Theorem 5.1) that

$$
\operatorname{det}\left(\frac{\partial^{2}}{\partial z_{j} \partial \bar{z}_{k}} \log K(z, \bar{z})\right)=c K(z, \bar{z})
$$

for some constant $c=c(\Omega)$. It follows that

$$
g(x, \bar{y})=c \cdot K(x, \bar{y})=c / a(x, \bar{y})
$$

and

$$
a(x, \bar{x})^{\alpha} d \mu(x)=c \cdot a(x, \bar{x})^{\alpha-1} \frac{d x}{\pi^{n}} .
$$

Therefore $A_{\alpha}^{2}=\widetilde{A}_{\alpha-1}^{2}, K_{\alpha}=\frac{1}{c} \widetilde{K}_{\alpha-1}$, and $B_{\alpha}=\widetilde{B}_{\alpha-1}$. Once again, both quantization procedures are (essentially) identical.

Example 4.8. $\Omega$ a hyperbolic planar domain with the Poincaré metric. Let $\lambda(z)$ be the metric coefficient for the Poincaré metric, as defined in Example 2.15. Thus the potential is $\Psi=-2 \log \lambda$ and $a=\lambda^{2}$. A brief computation shows that

$$
g=2 \lambda^{-2}=2 / a .
$$

Consequently,

$$
a^{\alpha} d \mu=2 a^{\alpha-1} \frac{d x}{\pi}
$$

and it follows that $A_{\alpha}^{2}=\widetilde{A}_{\alpha-1}^{2}, K_{\alpha}=\frac{1}{2} \widetilde{K}_{\alpha-1}$, and $B_{\alpha}=\widetilde{B}_{\alpha-1}$. Thus, the two quantization procedures are again identical (up to the shift of the index, $\alpha \leftrightarrow \alpha-1$ ).

Example 4.9. $\Omega$ is the one-dimensional cylinder, or, equivalently, the punctured plane $\mathbf{C} \backslash\{0\}$; and the Kähler metric is given by $d s^{2}=d z d \bar{z} / z \bar{z}$. In other words, $g_{1 \overline{1}}=g=1 / z \bar{z}$. Consequently,

$$
\int_{\mathbf{C} \backslash\{0\}}|z|^{2 k} \cdot a(z, \bar{z})^{\alpha} \frac{d z}{\pi}=\int_{\mathbf{C} \backslash\{0\}}|z|^{2 k+2} a(z, \bar{z})^{\alpha} d \mu(z),
$$


or $\left\|z_{k}\right\|_{\tilde{\alpha}}^{2}=\left\|z^{k+1}\right\|_{\alpha}^{2}$. Using the results from Example 2.16, we see that

$$
\widetilde{K}_{\alpha}(x, \bar{y})=\sum_{k \in \mathbf{Z}}(x \bar{y})^{k} /\left\|z^{k}\right\|_{\widetilde{\alpha}}^{2}=\frac{1}{x \bar{y}} \sum_{j \in \mathbf{Z}}(x \bar{y})^{j} /\left\|z^{j}\right\|_{\alpha}^{2}=\frac{1}{x \bar{y}} K_{\alpha}(x, \bar{y}) .
$$

Hence,

$$
\begin{aligned}
\frac{\left|\widetilde{K}_{\alpha}(x, \bar{y})\right|^{2}}{\widetilde{K}_{\alpha}(y, \bar{y})} a(x, \bar{x})^{\alpha} \frac{d x}{\pi} & =\frac{|y|^{2}}{|x y|^{2}} \frac{\left|K_{\alpha}(x, \bar{y})\right|^{2}}{K_{\alpha}(y, \bar{y})} a(x, \bar{x})^{\alpha} \frac{d x}{\pi} \\
& =\frac{\left|K_{\alpha}(x, \bar{y})\right|^{2}}{K_{\alpha}(y, \bar{y})} a(x, \bar{x})^{\alpha} d \mu(x)
\end{aligned}
$$

and it follows from the definition of the Berezin transform (4.2) that, once again, $\widetilde{B}_{\alpha}=B_{\alpha}$.

Remarkably, both approaches happen to yield an identical outcome ( $\widetilde{B}_{\alpha}=B_{\alpha}$ or $\left.\widetilde{B}_{\alpha}=B_{\alpha+1}\right)$ in all the four examples above. The reason is that, in these cases, the corresponding metrics have "constant curvature", i.e. (more precisely) they are Kähler-Einstein metrics. We will say more about this in the next section.

To get nontrivial examples, let us consider the Hartogs domains $\mathcal{D}_{F}$ from Section 3. To make things easier, we restrict ourselves to the case when $F(0)<+\infty$. Recall that the Kähler potential is given by $\Psi(x)=-\log a(x, \bar{x})$, where $a(x, \bar{x})=$ $F\left(\left|x_{1}\right|^{2}\right)-\left|x_{2}\right|^{2}$. According to the formula (3.17), the determinant $g$ is given by

$$
g(x, \bar{y})=\frac{F\left(x_{1} \bar{y}_{1}\right)^{2}}{a(x, \bar{y})^{3}} \cdot G\left(x_{1} \bar{y}_{1}\right)
$$

where

$$
G(t)=-\left(\frac{t F^{\prime}}{F}\right)^{\prime}
$$

We impose the following assumptions.

Assumption $\left(\mathrm{a}^{*}\right) . F$ is a meromorphic function on the disc BD, with no poles on $[0, B)$.

Assumption (b*). $F>0, F^{\prime}<0$, and $G=\left(-t F^{\prime} / F\right)^{\prime}>0$ on $[0, B)$.

Assumption (c*1). $|F(t)| \geq F(|t|) \quad \forall t \in B \mathbf{D}$, with equality iff $t \geq 0$.

Assumption $\left(\mathrm{c}^{*} 2\right)$. $|G(t)| \leq G(|t|)$ on $B \mathbf{D}$, and $\left(t G^{\prime} / G\right)^{\prime} \geq 0$ on $(0, B)$.

Assumption $\left(\mathrm{d}^{*}\right)$. There exist an infinite subset $E$ of the positive integers and a number $\gamma$ such that

$$
\sum_{k=0}^{\infty} t^{k} / c_{k}^{*}\left(F^{\alpha}\right)=(\alpha+1+\gamma) F(t)^{-\alpha} G(t) \quad \forall \alpha \in E .
$$

Here $c_{k}^{*}$ is defined as

$$
c_{k}^{*}\left(F^{\alpha}\right):=\int_{0}^{B} t^{k} F(t)^{\alpha} d t
$$

Let us put down some consequences of these assumptions. 
Proposition 4.10. For any function $G$ on $B \mathbf{D}$ which is continuous on $[0, B)$ and $C^{2}$ on $(0, B),\left(c^{*} 2\right)$ is equivalent to

$$
\left|G\left(x_{1} \bar{y}_{1}\right)\right|^{2} \leq G\left(\left|x_{1}\right|^{2}\right) G\left(\left|y_{1}\right|^{2}\right) \quad \forall x_{1}, y_{1} \in B \mathbf{D} .
$$

Proof. The condition $\left(t G^{\prime} / G\right)^{\prime} \geq 0$ means that the function $\phi_{G}(u)=\log G\left(e^{u}\right)$ is convex on $(0, B)$; that is,

$$
G\left(a^{2}\right) G\left(b^{2}\right) \geq G(a b)^{2} \quad \forall a, b \in(0, B) .
$$

By continuity, this inequality prevails also for $a=0$ or $b=0$. Therefore the first part of $\left(c^{*} 2\right)$ implies that

$$
\left|G\left(x_{1} \bar{y}_{1}\right)\right|^{2} \leq G\left(\left|x_{1} \bar{y}_{1}\right|\right)^{2} \leq G\left(\left|x_{1}\right|^{2}\right) G\left(\left|y_{1}\right|^{2}\right) \quad \forall x_{1}, y_{1} \in B \mathbf{D},
$$

which is (4.18).

Conversely, if (4.18) holds, then taking $\left|x_{1}\right|=\left|y_{1}\right|$ shows that $G(|t|) \geq|G(t)|$, and taking $x_{1}, y_{1} \in(0, B)$ shows that $\phi_{G}$ is convex, which is equivalent to $\left(t G^{\prime} / G\right)^{\prime} \geq 0$ on $(0, B)$.

Corollary 4.12. If $\left(\mathrm{a}^{*}\right),\left(\mathrm{b}^{*}\right),\left(\mathrm{c}^{*} 1\right)$ and $\left(\mathrm{c}^{*} 2\right)$ hold, then $\gamma(x \mid y) \leq 1$ for all $x, y$.

Proof. According to the last proposition,

$$
\left|G\left(x_{1} \bar{y}_{1}\right)\right|^{2} \leq G\left(x_{1} \bar{x}_{1}\right) G\left(y_{1} \bar{y}_{1}\right) .
$$

Moreover, by (3.25) and (b*),

$$
\frac{a(x, \bar{x}) a(y, \bar{y})}{|a(x, \bar{y})|^{2}} \leq \frac{F\left(x_{1} \bar{x}_{1}\right) F\left(y_{1} \bar{y}_{1}\right)}{\left|F\left(x_{1} \bar{y}_{1}\right)\right|^{2}} .
$$

Therefore by (4.15)

$$
\begin{aligned}
\gamma(x \mid y) & =\frac{|g(x, \bar{y})|^{2}}{g(x, \bar{x}) g(y, \bar{y})} \\
& =\frac{a(x, \bar{x})^{3} a(y, \bar{y})^{3}}{|a(x, \bar{y})|^{6}} \cdot \frac{\left|F\left(x_{1} \bar{y}_{1}\right)\right|^{4}}{F\left(x_{1} \bar{x}_{1}\right)^{2} F\left(y_{1} \bar{y}_{1}\right)^{2}} \cdot \frac{\left|G\left(x_{1} \bar{y}_{1}\right)\right|^{2}}{G\left(x_{1} \bar{x}_{1}\right) G\left(y_{1} \bar{y}_{1}\right)} \\
& \leq \frac{a(x, \bar{x}) a(y, \bar{y})}{|a(x, \bar{y})|^{2}} \\
& \leq 1
\end{aligned}
$$

The last inequality follows from $\left(\mathrm{b}^{*}\right),\left(\mathrm{c}^{*} 1\right)$ and Proposition 3.9.

Proposition 4.13. Let $F$ be such that $\mathcal{D}_{F}$ is a complete Reinhardt domain. Then the reproducing kernels for $\widetilde{A}_{\alpha}^{2}$ are given by

$$
\widetilde{K}_{\alpha}(x, \bar{y})=(\alpha+1) \sum_{k, l=0}^{\infty}\left(x_{1} \bar{y}_{1}\right)^{k}\left(x_{2} \bar{y}_{2}\right)^{l} \cdot\left(\begin{array}{c}
l+\alpha+1 \\
l
\end{array}\right) / c_{k}^{*}\left(F^{l+\alpha+1}\right),
$$

where $c_{k}^{*}$ are the coefficients (4.17). 
Proof. As has already been mentioned, it follows from the continuity and positivity of $a(x, \bar{x})^{\alpha}$ and from the mean value property of analytic functions that the evaluation functionals on $\widetilde{A}_{\alpha}^{2}$ are continuous at each point $x \in \mathcal{D}_{F}$. Thus we are in a position to apply Proposition 3.11. A computation similar to (3.36) shows that

$$
\begin{aligned}
\left\|x_{1}^{k} x_{2}^{l}\right\|^{2} & =\int_{\Omega}\left|z_{1}\right|^{2 k}\left|z_{2}\right|^{2 l}\left[F\left(\left|z_{1}\right|^{2}\right)-\left|z_{2}\right|^{2}\right]^{\alpha} \frac{d z}{\pi^{2}} \\
& =\int_{0}^{B} \int_{0}^{F\left(t_{1}\right)} t_{1}^{k} t_{2}^{l}\left[F\left(t_{1}\right)-t_{2}\right]^{\alpha} d t_{2} d t_{1} \\
& =\int_{0}^{B} \int_{0}^{1} t_{1}^{k} w^{l} F\left(t_{1}\right)^{l+\alpha+1}(1-w)^{\alpha} d w d t_{1} \\
& =\frac{l ! \alpha !}{(l+\alpha+1) !} c_{k}^{*}\left(F^{l+\alpha+1}\right)
\end{aligned}
$$

and an application of the formula (3.31) completes the proof.

Corollary 4.14. Let $F$ be such that $\mathcal{D}_{F}$ is a complete Reinhardt domain and assume that $\left(\mathrm{d}^{*}\right)$ holds. Then

$$
\widetilde{K}_{\alpha}(x, \bar{y})=(\alpha+1)[(\alpha+2)+\gamma(1-w(x, \bar{y}))] \cdot a(x, \bar{y})^{-\alpha} g(x, \bar{y}),
$$

where $w(x, \bar{y})=x_{2} \bar{y}_{2} / F\left(x_{1} \bar{y}_{1}\right)$.

Proof. Use (d*) to carry out the summation over $k$ in (4.20); this yields $\left(t_{i} \equiv x_{i} \bar{y}_{i}\right.$, $\left.w=t_{2} / F\left(t_{1}\right)\right)$

$$
\begin{aligned}
\frac{\widetilde{K}_{\alpha}(x, \bar{y})}{\alpha+1} & =\sum_{l=0}^{\infty}\left(\begin{array}{c}
l+\alpha+1 \\
l
\end{array}\right) \cdot t_{2}^{l}(l+\alpha+2+\gamma) G\left(t_{1}\right) F\left(t_{1}\right)^{-l-\alpha-1} \\
& =G\left(t_{1}\right) F\left(t_{1}\right)^{-\alpha-1} \sum_{l=0}^{\infty}\left[(\alpha+2)\left(\begin{array}{c}
l+\alpha+2 \\
l
\end{array}\right)+\gamma\left(\begin{array}{c}
l+\alpha+1 \\
l
\end{array}\right)\right] \cdot \frac{t_{2}^{l}}{F\left(t_{1}\right)^{l}} \\
& =G\left(t_{1}\right) F\left(t_{1}\right)^{-\alpha-1} \cdot\left[(\alpha+2)(1-w)^{-\alpha-3}+\gamma(1-w)^{-\alpha-2}\right] \\
& =G\left(t_{1}\right) F\left(t_{1}\right)^{2}\left[(\alpha+2) a^{-\alpha-3}+\gamma \cdot(1-w) a^{-\alpha-3}\right] \\
4.23) \quad & g \cdot a^{-\alpha}[(\alpha+2)+\gamma(1-w)] \quad(\text { by }(4.15)),
\end{aligned}
$$

which is the desired formula.

Theorem 4.15. Assume that $\left(\mathrm{a}^{*}\right),\left(\mathrm{b}^{*}\right),\left(\mathrm{c}^{*} 1\right),\left(\mathrm{c}^{*} 2\right)$ and $\left(\mathrm{d}^{*}\right)$ are satisfied. Then the algebra $\mathcal{A}$ constructed from the algebras $\mathcal{A}_{h}$ of covariant symbols for $\widetilde{A}_{\alpha}^{2}, \alpha \in$ $E$, is a quantization of $\left(\mathcal{D}_{F}, \omega_{F}\right)$ satisfying the weak form of the correspondence principle.

Proof. By $\left(\mathrm{a}^{*}\right)$, the function $a(x, \bar{y})=F\left(x_{1} \bar{y}_{1}\right)-x_{2} \bar{y}_{2}$ satisfies (E*). By (b*), $\left(c^{*} 1\right)$ and Proposition 3.9(i), all points of $\mathcal{D}_{F}$ are regular, i.e. $\left(\mathrm{C}^{*} 1\right)$ is satisfied. By Corollary $4.12, \gamma(x \mid y) \leq 1$ on $\Omega \times \Omega$, i.e. $\left(\mathrm{C}^{*} 2\right)$ is fulfilled. Finally, by $\left(\mathrm{c}^{*} 1\right)$ and Proposition 3.9(ii),

$$
|w(x, \bar{y})|^{2}=\frac{\left|x_{2} \bar{y}_{2}\right|^{2}}{\left|F\left(x_{1} \bar{y}_{1}\right)\right|^{2}} \leq \frac{F\left(\left|x_{1}\right|^{2}\right) F\left(\left|x_{2}\right|^{2}\right)}{\left|F\left(x_{1} \bar{y}_{1}\right)\right|^{2}} \leq 1,
$$


and the formula (4.22) in the last corollary implies that $\left(\mathrm{A}^{*}\right)$ holds, with $B(x, \bar{y})=$ $3+(1-w) \gamma$ and $C(x, \bar{y}, \alpha)=2+\gamma(1-w)$. Consequently, an application of Theorem 4.5 is legitimate, and the assertion follows.

Example 4.16. $F(t)=(1-t)^{p}, p>0 ; B=1$. This is the "complex ellipsoid" from Example 3.19. The conditions $\left(\mathrm{a}^{*}\right),\left(\mathrm{b}^{*}\right)$ and $\left(\mathrm{c}^{*} 1\right)$ were already checked there, so we need only worry about $\left(c^{*} 2\right)$ and $\left(d^{*}\right)$. Since

$$
G(t)=\frac{p}{(1-t)^{2}}
$$

the triangle inequality immediately implies that $|G(t)| \leq G(|t|)$; moreover,

$$
\left(\frac{t G^{\prime}}{G}\right)^{\prime}=\left(\frac{2 t}{1-t}\right)^{\prime}=\frac{2}{(1-t)^{2}} \geq 0
$$

so $\left(c^{*} 2\right)$ is fulfilled. Further,

$$
c_{k}^{*}\left(F^{\alpha}\right)=\int_{0}^{1} t^{k}(1-t)^{\alpha p} d t=\frac{k !(\alpha p) !}{(k+\alpha p+1) !}
$$

whence

$$
\begin{aligned}
\sum_{k=0}^{\infty} t^{k} / c_{k}^{*}\left(F^{\alpha}\right) & =(\alpha p+1) \sum_{k=0}^{\infty}\left(\begin{array}{c}
k+\alpha p+1 \\
k
\end{array}\right) t^{k} \\
& =(\alpha p+1)(1-t)^{-\alpha p-2}=\left(\alpha+\frac{1}{p}\right) F^{-\alpha} G .
\end{aligned}
$$

Therefore $\left(\mathrm{d}^{*}\right)$ holds as well (with $\gamma=\frac{1}{p}-1$ ), and Theorem 4.5 applies.

By Corollary 4.14, the reproducing kernels are given by

$$
\widetilde{K}_{\alpha}(x, \bar{y})=(\alpha+1)\left[(\alpha+2)+\left(\frac{1}{p}-1\right)(1-w)\right] \cdot a(x, \bar{y})^{-\alpha-3} \cdot p\left(1-t_{1}\right)^{2 p-2} .
$$

Example 4.17. $F(t)=e^{-t}, B=+\infty$. This is the Springer domain from Example 3.20. Again, only $\left(\mathrm{c}^{*} 2\right)$ and $\left(\mathrm{d}^{*}\right)$ need to be checked. Since

$$
G(t)=1
$$

$\left(\mathrm{c}^{*} 2\right)$ is trivial. As for $\left(\mathrm{d}^{*}\right)$, we have

$$
c_{k}^{*}\left(F^{\alpha}\right)=\int_{0}^{+\infty} t^{k} e^{-\alpha t} d t=k ! / \alpha^{k+1},
$$

so

$$
\sum_{k=0}^{\infty} t^{k} / c_{k}^{*}\left(F^{\alpha}\right)=\alpha e^{\alpha t}=\alpha F^{-\alpha} G .
$$

Thus $\left(\mathrm{d}^{*}\right)$ holds with $\gamma=-1$, and Theorem 4.5 is applicable. 
Example 4.18. $F(t)=1 /(t+1)^{p}, p$ an integer $\geq 2, B=+\infty$ (the domain from Example 3.23). Again, only $\left(\mathrm{c}^{*} 2\right)$ and $\left(\mathrm{d}^{*}\right)$ have to be checked. This time we will start with $\left(\mathrm{d}^{*}\right)$ :

$$
\begin{aligned}
c_{k}^{*}\left(F^{\alpha}\right) & =\int_{0}^{+\infty} t^{k}(1+t)^{-p \alpha} d t \\
& =\int_{0}^{1}\left(\frac{1}{s}-1\right)^{k} s^{p \alpha-2} d s \quad\left(s=\frac{1}{1+t}\right) \\
& =\int_{0}^{1} s^{p \alpha-k-2}(1-s)^{k} d s= \begin{cases}+\infty & \text { if } k>p \alpha-2, \\
\frac{k !(p \alpha-k-2) !}{(p \alpha-1) !} & \text { if } 0 \leq k \leq p \alpha-2 .\end{cases}
\end{aligned}
$$

Hence,

$$
\begin{aligned}
\sum_{k=0}^{\infty} t^{k} / c_{k}^{*}\left(F^{\alpha}\right) & =(p \alpha-1) \sum_{k=0}^{p \alpha-2}\left(\begin{array}{c}
p \alpha-2 \\
k
\end{array}\right) t^{k}=(p \alpha-1)(1+t)^{p \alpha-2} \\
& =\left(\alpha-\frac{1}{p}\right) F^{-\alpha} \cdot \frac{p}{(1+t)^{2}}=\left(\alpha-\frac{1}{p}\right) F^{-\alpha} G
\end{aligned}
$$

which means that $\left(\mathrm{d}^{*}\right)$ is fulfilled with $\gamma=-1-\frac{1}{p}$.

On the other hand, the triangle inequality implies that $\left(\mathrm{c}^{*} 2\right)$ cannot hold (it even holds with the opposite sign!). Thus we must prove $\left(\mathrm{C}^{*} 2\right)$ - which is the only thing in the proof of Theorem 4.5 that $\left(\mathrm{c}^{*} 2\right)$ was needed for - directly. Observe that

$$
G(t)=\frac{p}{(1+t)^{2}}=p \cdot F(t)^{2 / p}
$$

Using (4.15) and (4.19), it follows that

$$
\begin{aligned}
\frac{|g(x, \bar{y})|^{2}}{g(x, \bar{x}) g(y, \bar{y})} & =\frac{a(x, \bar{x})^{3} a(y, \bar{y})^{3}}{|a(x, \bar{y})|^{6}} \cdot\left[\frac{\left|F\left(x_{1} \bar{y}_{1}\right)\right|^{2}}{F\left(x_{1} \bar{x}_{1}\right) F\left(y_{1} \bar{y}_{1}\right)}\right]^{2+2 / p} \\
& \leq\left[\frac{a(x, \bar{x}) a(y, \bar{y})}{|a(x, \bar{y})|^{2}}\right]^{3-2-2 / p} \\
& =e^{(1-2 / p) \psi(x \mid y)} \leq 1
\end{aligned}
$$

since $p \geq 2$ by hypothesis and $\psi \leq 0$ by $\left(\mathrm{C}^{*} 1\right)$. Hence, Theorem 4.5 can be applied.

It is interesting to see what happens for $p=1$. In that case, we only have, by virtue of the last inequality,

$$
\gamma(x \mid y) e^{\psi(x \mid y)} \leq 1
$$

This suggests trying to make things work under the weaker assumption

$$
\exists \beta>0: \quad \gamma(x \mid y) e^{\beta \psi(x \mid y)} \leq 1 \quad \forall x, y \in \Omega
$$

in the place of $\left(\mathrm{C}^{*} 2\right)$. It turns out that, indeed, the analogues of Theorems 4.1 and 4.3 still hold, and so does the analogue of Theorem 4.4 . 
Theorem 4.19. Assume that ( $\left.\mathrm{E}^{*}\right),\left(\mathrm{A}^{*}\right)$ and (4.24) are satisfied and $y \in \Omega$ is regular. Let $f$ be any function on $\Omega$ which is $C^{3}$ in a neighbourhood of $y$ and for which the integral defining the Berezin transform

$$
\widetilde{B}_{\alpha} f(y)=\int_{\Omega} f(x) \frac{\left|\widetilde{K}_{\alpha}(x, \bar{y})\right|^{2}}{\widetilde{K}_{\alpha}(y, \bar{y})} \cdot a(x, \bar{x})^{\alpha} \frac{d x}{\pi^{n}}
$$

converges absolutely for $\alpha=\alpha_{1}$. Then the integral exists for all $\alpha \geq \alpha_{1}, \alpha \in E$, and

$$
\widetilde{B}_{\alpha} f(y)=f(y)+\alpha^{-1} \Delta f(y)+o\left(\alpha^{-1}\right) \quad \text { as } \alpha \rightarrow+\infty,
$$

where $\Delta$ is the Laplace-Beltrami operator on $\Omega$.

Theorem 4.20. Assume that $\left(\mathrm{E}^{*}\right),\left(\mathrm{A}^{*}\right),\left(\mathrm{C}^{*} 1\right)$ and $(4.24)$ are fulfilled, and let $f, g \in \widetilde{\mathcal{A}}$, where $\widetilde{\mathcal{A}} \subset \mathcal{A}$ is defined by the conditions $(\widetilde{\mathcal{A}} 1)$ and $(\widetilde{\mathcal{A}} 2)$. Then

$$
(f * g)(h \mid z)=f(0 \mid z) g(0 \mid z)+o(1),
$$

and

$$
\frac{1}{h}(f * g-g * f)(h \mid z)=\frac{1}{i}\{f, g\}(0 \mid z)+o(1)
$$

as $\alpha \rightarrow+\infty, \alpha \in E$, where $\{\cdot, \cdot\}$ stands for the Poisson bracket.

Theorem 4.21. Assume that $\left(\mathrm{E}^{*}\right),\left(\mathrm{A}^{*}\right),\left(\mathrm{C}^{*} 1\right)$ and $(4.24)$ are satisfied. Then for any two distinct points $z_{0}, z_{1} \in \Omega$ there exists a function $f \in \widetilde{\mathcal{A}}$ such that $f\left(0 \mid z_{0}\right) \neq f\left(0 \mid z_{1}\right)$.

The proofs of Theorems 4.19 and 4.20 are but obvious modifications of those of Theorems 4.1 and 4.3, so we omit them. In the proof of Theorem 4.21, the second line of the estimate (4.14) has to be replaced by (we omit the factor $4 \alpha^{2 n}$ )

$$
\begin{aligned}
|a(x, \bar{y})|^{-2\left(\alpha-\alpha_{1}\right)}|g(x, \bar{y})|^{2} & \leq g(x, \bar{x}) g(y, \bar{y}) \cdot a(x, \bar{x})^{-\beta} a(y, \bar{y})^{-\beta}|a(x, \bar{y})|^{-2\left(\alpha-\alpha_{1}-\beta\right)} \\
& \leq g(x, \bar{x}) g(y, \bar{y}) \cdot a(x, \bar{x})^{\alpha_{1}-\alpha} a(y, \bar{y})^{\alpha_{1}-\alpha}
\end{aligned}
$$

for $\alpha>\alpha_{1}+\beta$, which is a consequence of (4.24) and ( $\left.\mathrm{C}^{*} 1\right)$. Also, the function $\epsilon(h)$ must be chosen to vanish already for $1 / h<\alpha_{1}+\beta$, instead of for $1 / h<\alpha_{1}$. The rest of the proof works without change.

\section{CONCluding REMARKS}

5.1. Biholomorphic invariance. Suppose that $(\Omega, \omega)$ and $(\hat{\Omega}, \hat{\omega})$ are two Kähler manifolds which are holomorphically equivalent, that is, there exists a biholomorphic mapping $\phi$ of $\Omega$ onto $\hat{\Omega}$ which sends $\omega$ into $\hat{\omega}$, i.e. for which

$$
\left(g_{j \bar{k}}\right)=J_{\phi} \cdot\left(\hat{g}_{j \bar{k}} \circ \phi\right) \cdot J_{\phi}^{*}
$$

where $J_{\phi}=\left(\partial \phi_{j} / \partial x_{i}\right)_{i, j=1}^{n}$ is the complex Jacobian matrix of $\phi$. In terms of Kähler potentials, (5.1) amounts to

$$
\frac{\partial^{2}}{\partial z_{j} \partial \bar{z}_{k}}(\hat{\Psi} \circ \phi-\Psi)=0 \quad \text { on } \Omega .
$$


Thus, if we have chosen $\Psi$ as a potential for $\omega$, then $\Psi \circ \phi^{-1}=: \hat{\Psi}$ can serve as a potential for $\hat{\omega}$, and the function

$$
\hat{a}(\phi(x), \overline{\phi(y)}):=a(x, \bar{y})
$$

then satisfies the assumption (E) whenever $a(x, \bar{y})$ satisfies it. Since, by (5.1), $g=\left|\operatorname{det} J_{\phi}\right|^{2} \cdot(\hat{g} \circ \phi)$, we have

$$
d \hat{\mu}(\phi(x))=d \mu(x)
$$

and it follows that the mapping $f \mapsto f \circ \phi$ is a Hilbert space isomorphism of $A_{\alpha}^{2}(\hat{\Omega})$ onto $A_{\alpha}^{2}(\Omega)$, for any $\alpha>0$. Consequently,

$$
\hat{K}_{\alpha}(\phi(x), \overline{\phi(y)})=K_{\alpha}(x, \bar{y})
$$

and the definition of the Berezin transform implies that

$$
\left(\hat{B}_{\alpha} F\right) \circ \phi=B_{\alpha}(F \circ \phi)
$$

for all $\alpha>0$ and any function $F$ on $\hat{\Omega}$. In other words, we see that the Berezin transform as well as the whole quantization procedure are invariant under biholomorphic equivalence.

5.2. Automorphisms and invariant metrics. Denote by $\operatorname{Aut}(\Omega)$ the group of all biholomorphic automorphisms of $\Omega$. A Kähler form $\omega=\sum_{j, k} g_{j \bar{k}} d x_{j} \wedge d \bar{x}_{k}$ is said to be invariant if

$$
g_{j \bar{k}}=J_{\phi} \cdot\left(g_{j \bar{k}} \circ \phi\right) \cdot J_{\phi}^{*} \quad \text { on } \Omega
$$

for all $\phi \in \operatorname{Aut}(\Omega)$, i.e. when $\omega$ is invariant under all biholomorphic automorphisms of $\Omega$. In view of (5.2), a sufficient condition for invariance is that there exist for each $\phi \in \operatorname{Aut}(\Omega)$ a non-vanishing holomorphic function $H_{\phi}$ on $\Omega$ such that

$$
\Psi \circ \phi=\Psi-\log \left|H_{\phi}\right|^{2}
$$

or, in terms of $a=e^{-\Psi}$,

$$
a \circ \phi=a \cdot\left|H_{\phi}\right|^{2} \quad \text { on } \Omega \text {. }
$$

If $\Omega$ is simply connected, this condition is also necessary. In view of the observations made in the previous paragraph, invariance of $\omega$ implies that the Berezin transform as well as the whole quantization procedure commute (in the obvious sense) with the action of $\operatorname{Aut}(\Omega)$.

Invariant forms are clearly very aesthetic objects to deal with. According to familiar facts from Riemannian geometry, the Kähler forms from Examples 2.14 (bounded symmetric domains), 2.15 (hyperbolic plane domains) and 2.16 (the punctured plane) are invariant, whereas that from Example $2.13\left(\mathbf{C}^{n}\right)$ is invariant only for a certain subgroup of $\operatorname{Aut}(\Omega)$ (the rigid motions group). For the domains appearing in the examples in Section 3, the automorphism groups seem to be either unknown or trivial (i.e. reduced to the rotations around coordinate axes, cf. Sunada 
[Su] and the theorem of H. Cartan [BM, Theorems I.1 and I.8]), with the exception of Example 3.19 (complex ellipsoids). We shall show that for complex ellipsoids, the Kähler form $\omega_{F}$ is invariant.

Recall that the automorphisms of the complex ellipsoid $\mathcal{D}_{F}, F(t)=(1-t)^{p}$, $p \neq 1$, are given by

$$
\phi(x)=\left(\frac{x_{1}-b}{1-\bar{b} x_{1}}, \varepsilon \frac{\left(1-|b|^{2}\right)^{p / 2}}{\left(1-\bar{b} x_{1}\right)^{p}} x_{2}\right), \quad x=\left(x_{1}, x_{2}\right) \in \mathcal{D}_{F},
$$

where $b \in \mathbf{D}$ and $|\varepsilon|=1$. The function $a$ is given by $a(x)=\left(1-\left|x_{1}\right|^{2}\right)^{p}-\left|x_{2}\right|^{2}$; thus,

$$
\begin{aligned}
a \circ \phi(x) & =\left[\frac{\left(1-\left|x_{1}\right|^{2}\right)\left(1-|b|^{2}\right)}{\left|1-\bar{b} x_{1}\right|^{2}}\right]^{p}-\frac{\left(1-|b|^{2}\right)^{p}}{\left|1-\bar{b} x_{1}\right|^{2 p}}\left|x_{2}\right|^{2} \\
& =\frac{\left(1-|b|^{2}\right)^{p}}{\left|1-\bar{b} x_{1}\right|^{2 p}} \cdot a(x),
\end{aligned}
$$

so (5.4) is satisfied with

$$
H_{\phi}(x)=\frac{\left(1-|b|^{2}\right)^{p / 2}}{\left(1-\bar{b} x_{1}\right)^{p}}
$$

which proves the invariance of $\omega_{F}$. Incidentally, it can be shown that the function $H_{\phi}$ is equal to $\left(\operatorname{det} J_{\phi}\right)^{\frac{p}{p+2}}$; it follows from this that $a(x)$ obeys the same transformation law as

$$
\widetilde{K}_{0}(x, \bar{x})^{-p /(p+2)}
$$

( $\widetilde{K}_{0}$ is the notation from Section 4 - the Bergman kernel function with respect to the Lebesgue measure). Hence $\widetilde{K}_{0} \cdot a^{\frac{p+2}{p}}$ is invariant under $\operatorname{Aut}\left(\mathcal{D}_{F}\right)$. Now with the usual notation

$$
w(x)=\frac{\left|x_{2}\right|^{2}}{F\left(\left|x_{1}\right|^{2}\right)}=1-\frac{a(x)}{\left(1-\left|x_{1}\right|^{2}\right)^{p}}, \quad x \in \mathcal{D}_{F},
$$

formula (5.5) reads

$$
w \circ \phi=w .
$$

Conversely, it can be shown that any two points $x, y \in \mathcal{D}_{F}$ with $w(x)=w(y)$ satisfy $y=\phi(x)$ for some $\phi \in \operatorname{Aut}\left(\mathcal{D}_{F}\right)$. In other words, the level-sets of $w(x)$ are the orbits of $\operatorname{Aut}\left(\mathcal{D}_{F}\right)$; consequently,

$$
\widetilde{K}_{0}(x, \bar{x}) \cdot a(x, \bar{x})^{(p+2) / p}
$$

must be a function of $w(x)$ only. Similar argument shows that the functions

$$
K_{\alpha}(x, \bar{x}) a(x, \bar{x})^{\alpha}, \quad \widetilde{K}_{\alpha}(x, \bar{x}) a(x, \bar{x})^{\alpha} / g(x, \bar{x}), \quad a(x, \bar{x})^{(p+2) / p} g(x, \bar{x})
$$

are likewise invariant under $\operatorname{Aut}\left(\mathcal{D}_{F}\right)$ and, hence, are functions of $w(x)$ only (for each fixed $\alpha$ ). This, of course, agrees with our formulas from Examples 3.19 and 4.16. Similar comments can be made for a general Kähler metric invariant under $\operatorname{Aut}(\Omega)$. 
5.3. Poincaré metrics. In the Examples 4.6-4.9, we have seen that both Berezin transforms $B_{\alpha}$ and $\widetilde{B}_{\alpha}$ happened to amount to the same thing. Let us consider this more closely. Recall that the Ricci tensor $R_{j \bar{k}}$ of the Kähler metric $\sum g_{j \bar{k}} d x_{j} d \bar{x}_{k}$ is defined by

$$
R_{j \bar{k}}=\frac{\partial^{2} \log g}{\partial x_{j} \partial \bar{x}_{k}} .
$$

If the Ricci tensor is a constant multiple of the fundamental tensor $g_{j \bar{k}}$,

$$
R_{j \bar{k}}=\kappa \cdot g_{j \bar{k}}
$$

then the metric is called a Kähler-Einstein metric. Suppose that this is the case. If $\kappa \neq 0$, then

$$
\Psi(x)=\frac{1}{\kappa} \log g(x)
$$

can serve as the Kähler potential, and the corresponding function $a$ equals

$$
a(x)=g(x)^{-1 / \kappa} .
$$

Thus $a(x)^{\alpha} d \mu(x)=a(x)^{\alpha-\frac{1}{\kappa}} d x / \pi^{n}$, and, consequently,

$$
B_{\alpha}=\widetilde{B}_{\alpha-1 / \kappa},
$$

i.e. the two Berezin transforms, as well as both quantization procedures, are (in the obvious sense) identical.

When $\kappa=0$ and $\Omega$ is simply connected, the equations

$$
\frac{\partial^{2} \log g}{\partial x_{j} \partial \bar{x}_{k}}=0
$$

imply that $\log g$ is the real part of an analytic function, i.e. $g=|H|^{2}$ for some zero-free analytic function $H$ on $\Omega$. Thus $d \mu(x)=|H(x)|^{2} d x / \pi^{n}$, and it follows that the mapping

$$
f \mapsto f \cdot H
$$

is a Hilbert space isomorphism of $A_{\alpha}^{2}$ onto $\widetilde{A}_{\alpha}^{2}$, for any $\alpha>0$. Consequently,

$$
\widetilde{K}_{\alpha}(x, \bar{y})=H(x) \overline{H(y)} K_{\alpha}(x, \bar{y}),
$$

hence

$$
\frac{\left|\widetilde{K}_{\alpha}(x, \bar{y})\right|^{2}}{\widetilde{K}_{\alpha}(y, \bar{y})} d x=\frac{\left|K_{\alpha}(x, \bar{y})\right|^{2}}{K_{\alpha}(y, \bar{y})} d \mu(x),
$$

and it follows that

$$
\widetilde{B}_{\alpha}=B_{\alpha}
$$

for all $\alpha$, and we arrive at the same conclusion as in the preceding paragraph. (We won't discuss the case when $\kappa=0$ and $\Omega$ is not simply connected.)

Apparently, Kähler-Einstein metrics are a very canonical object and deserve further study regarding the possibility of applying the Berezin quantization to them. When $\Omega$ is a bounded pseudoconvex domain in $\mathbf{C}^{n}$, a deep theorem of Cheng and 
Yau ([ChY], [BFG, Chapter 11], [MY]) asserts that there exists a unique KählerEinstein metric (called the Poincaré metric) $\sum_{j, k} g_{j \bar{k}} d x_{j} d \bar{x}_{k}$ on $\Omega$ for which (5.8) holds with, say, $\kappa=1$ and which satisfies

$$
g=\operatorname{det}\left(g_{j \bar{k}}\right)=\frac{1}{u(z)^{n+1}},
$$

where the function $u(z)$ is positive on $\Omega$ and has a zero of order exactly one on $\partial \Omega$. Note that, since the Kähler-Einstein condition (5.8) is invariant under biholomorphic maps, it follows from the uniqueness of the Poincaré metric that it is invariant under $\operatorname{Aut}(\Omega)$ in the sense discussed above.

If $\Omega$ is a bounded pseudoconvex domain in $\mathbf{C}^{n}$ and $\omega$ is the Kähler form corresponding to the Poincaré metric (with $\kappa=1$ ), then

$$
\Psi=\log g
$$

can serve as the potential; the corresponding function $a=e^{-\Psi}$ is thus equal to

$$
a(z)=u(z)^{n+1}
$$

Our assumption $\left(\mathrm{E}^{\prime}\right)$ now requires that $u(z)$ be extendable to a sesquimeromorphic function $u(x, \bar{y})$ on $\Omega \times \Omega$. Although the function $u(z)$ is known to be $C^{\omega}$ on $\Omega$ $[\mathrm{DK}],{ }^{11}$ it seems unclear whether such an extension exists; it is certainly tempting to conjecture that it does, and, likewise, to conjecture that the condition $\left(\mathrm{A}^{\prime \prime}\right)$ is fulfilled for the corresponding reproducing kernels. In view of (5.8), (2.23), and Corollary 2.6, $c_{n}=1$ and

$$
B(x, \bar{x})=-\frac{3}{2} \Delta \log g(x)=-\frac{3}{2} \sum_{j, k} g^{\bar{k} j} g_{j \bar{k}}=-\frac{3}{2} n,
$$

which is independent of $x$, so $\left(\mathrm{A}^{\prime \prime}\right)$ reads just

$$
K_{\alpha}(x, \bar{y}) a(x, \bar{y})^{\alpha}=\alpha^{n}-\frac{3}{2} n \alpha^{n-1}+O\left(\alpha^{n-2}\right)
$$

with the $O$ uniform in $x$ for each fixed $y$. Though it may be too far-fetched to expect this to hold, we conjecture that, in the analogy with the situation in Example 2.15 (formula (2.44)),

$$
K_{\alpha}(x, \bar{y}) a(x, \bar{y})^{\alpha}=P(\alpha)+O\left(\gamma^{\alpha}\right) \quad \forall x, y \in \Omega
$$

for any bounded pseudoconvex domain $\Omega \subset \mathbf{C}^{n}$ with the Poincaré metric, where $P(\alpha)$ is a polynomial in $\alpha$ with leading term $\alpha^{n}$ and $0<\gamma=\gamma(x, \bar{y})<1$.

Example 5.4. For any $c \leq 0$,

$$
\mathcal{D}_{c}=\left\{z \in \mathbf{C}^{2}: \operatorname{Re} z_{1}>\left|z_{2}\right|^{2}+c \log \left|z_{2}\right|^{2}\right\}
$$

\footnotetext{
${ }^{11}$ For strongly pseudoconvex domains with $C^{5}$ boundary, this was already proved in [ChY].
} 
is an (unbounded) strongly pseudoconvex domain in $\mathbf{C}^{2}$, and

$$
\left(\operatorname{Re} z_{1}-\left|z_{2}\right|^{2}-c \log \left|z_{2}\right|^{2}\right)^{-3}
$$

serves as a potential of the Poincaré metric on $\mathcal{D}_{c} \cdot{ }^{12}$ (For $c>0$, the metric is still Kähler-Einstein, but it degenerates at points with $z_{2}=0$.) What are the reproducing kernels?

For $c=0, \mathcal{D}_{0}$ is just the unbounded (tube domain) realization of the unit ball (via the Cayley transform), so we get nothing new; for general $c$, the behaviour of the kernels is unknown.

5.5. Curvature. The number

$$
R:=\sum_{j, k} g^{\bar{k} j} R_{j \bar{k}}=\sum_{j, k} g^{\bar{k} j} \frac{\partial^{2} \log g}{\partial z_{j} \partial \bar{z}_{k}}=\Delta \log g \quad \text { (by 2.23) }
$$

is known as the scalar curvature of the Kähler form $\omega$ [BFG, Section V.2]. From Corollaries 2.6 and 4.2, we therefore obtain the following geometric property which is implied by our assumptions:

Proposition 5.6. Assume that $\left(\mathrm{E}^{\prime}\right),\left(\mathrm{A}^{\prime}\right)$ and $\left(\mathrm{C}^{\prime}\right) \quad\left(\right.$ or $\left(\mathrm{E}^{*}\right),\left(\mathrm{A}^{*}\right),\left(\mathrm{C}^{*} 1\right)$ and $\left.\left(\mathrm{C}^{*} 2\right)\right)$ are fulfilled. Then the scalar curvature $R(x)$ extends to a bounded sesquianalytic function $R(x, \bar{y})$ on $\Omega \times \Omega$.

The above corollaries can also be used for obtaining formulas for the scalar curvature in those cases where we were able to compute the reproducing kernels explicitly (Examples 2.13 and 2.14, 3.19-3.21, 3.23, 3.29-3.30, and 4.16-4.18). For the domains $\mathcal{D}_{F}$ in Sections 3 and 4 , the curvature $R(z)$ depends, remarkably, only on the expression $w(z)=\left|z_{2}\right|^{2} / F\left(\left|z_{1}\right|^{2}\right)$. (For the complex ellipsoids in Example 3.19 this, of course, follows directly from (5.6) and from the fact that curvature is a biholomorphic invariant.)

5.7. Exact vs. asymptotic formulas for the reproducing kernels. Examples 3.19 and 3.30 (with $p=1$ ) and 2.13, 2.14 were the only ones we have encountered for which the assumption $\left(\mathrm{A}^{\prime}\right)$ was fulfilled with $B(x, \bar{y})$ and $C(x, \bar{y}, \alpha)$ constant:

$$
K_{\alpha}(x, \bar{y})=a(x, \bar{y})^{-\alpha} \cdot\left(\text { a polynomial in } \alpha \text { with leading term } \alpha^{n}\right) .
$$

It turns out, moreover, that Example 3.30 is actually only a "consequence" of Example 3.19. Let us explain this in more detail.

We will agree to call a set $E \subset \Omega$ negligible if $E$ has Lebesgue measure zero and for any open set $U$ intersecting $E$, every square-integrable (with respect to the Lebesgue measure) analytic function on $U \backslash E$ extends to an analytic function on all of $U$. Observe that the positivity and continuity of $a(x, \bar{x})$ implies that for any negligible set $E \subset \Omega$ and any $\alpha>0$, the restriction mapping $\left.f \mapsto f\right|_{\Omega \backslash E}$ is an isomorphism of $A_{\alpha}^{2}\left(\Omega, a^{\alpha} d \mu\right)$ onto $A_{\alpha}^{2}\left(\Omega \backslash E, a^{\alpha} d \mu\right)$. A domain $\hat{\Omega}$ will be said to be subordinate to $\Omega$ if there exist negligible sets $E \subset \Omega, \hat{E} \subset \hat{\Omega}$ and a holomorphic

\footnotetext{
${ }^{12}$ Note that, as $\mathcal{D}_{c}$ is unbounded, the Cheng-Yau theorem does not apply in this case. It seems to be rather unclear what is the situation regarding the existence of Kähler-Einstein metrics on unbounded domains.
} 
mapping $\phi: \hat{\Omega} \rightarrow \Omega$ such that $\phi$ is a biholomorphism of $\hat{\Omega} \backslash \hat{E}$ onto $\Omega \backslash E$. (N.B.: $\phi$ is defined on all of $\hat{\Omega}$.) It follows from the change-of-variable formula

$$
\int_{\hat{\Omega} \backslash \hat{E}}|f \circ \phi|^{2}(a \circ \phi)^{\alpha} d \hat{\mu}=\int_{\Omega \backslash E}|f|^{2} a^{\alpha} d \mu
$$

and from the above observation that the mapping $f \mapsto f \circ \phi$ is a Hilbert space isomorphism of $A_{\alpha}^{2}\left(\Omega, a^{\alpha} d \mu\right)$ onto $A_{\alpha}^{2}\left(\hat{\Omega}, \hat{a}^{\alpha} d \hat{\mu}\right)$, granted we define $\hat{a}(x, \bar{y})$ on $\hat{\Omega}$ by

$$
\hat{a}(x, \bar{y}):=a(\phi(x), \overline{\phi(y)})
$$

(and, consequently, $\hat{g}_{j \bar{k}}$ by $\left(\hat{g}_{j \bar{k}}\right)=J_{\phi} \cdot\left(g_{j \bar{k}} \circ \phi\right) \cdot J_{\phi}^{*}$, as in (5.1), so that $d \hat{\mu}(x)=$ $d \mu(\phi(x)))$. The reproducing kernels therefore satisfy

$$
\hat{K}_{\alpha}(x, \bar{y})=K_{\alpha}(\phi(x), \overline{\phi(y)})
$$

and it's immediate that the validity of $(5.11)$ for $\hat{K}_{\alpha}$ follows from the validity of (5.11) for $K_{\alpha}$ (i.e. if things go well on $\Omega$, they also go well on $\hat{\Omega}$ ).

Now apply this argument to $\Omega=\mathcal{D}_{F_{0}}$ and $\hat{\Omega}=\mathcal{D}_{F}$, where $F_{0}(t)=(1-t)^{p}$ and $F(t)=F_{0}(t) / t$. The mapping

$$
\phi:\left(x_{1}, x_{2}\right) \in \mathcal{D}_{F} \longmapsto\left(x_{1}, x_{1} x_{2}\right) \in \mathcal{D}_{F_{0}}
$$

is a biholomorphism of $\mathcal{D}_{F} \backslash \hat{E}$ onto $\mathcal{D}_{F_{0}} \backslash E$, where the subsets $E, \hat{E}$ consist of all points $x$ with $x_{1}=0$. A simple argument based on the Laurent expansions in the $x_{2}$-variable shows that both $E$ and $\hat{E}$ are negligible. Finally, the function $\hat{a}(x, \bar{y})$ given by (5.12) differs from $a_{F}(x, \bar{y})$ only by a multiplicative factor $x_{1} \bar{y}_{1}$, which can be disposed of by using an equivalent Kähler potential on $\Omega$ (cf. Proposition 2.7). Thus, the validity of $\left(\mathrm{A}^{\prime}\right)$ on the complex ellipsoid $\mathcal{D}_{F_{0}}$ (Example 3.19) implies its validity for $\mathcal{D}_{F}$ (Example 3.30). In particular, for $p=1$, the validity of (5.11) for the domain $\mathcal{D}_{F}, F=(1-t) / t$, follows from its validity for the unit ball in $\mathbf{C}^{2}$.

Remark 5.8. Slightly adapted (in the spirit of Examples 2.15 and 2.16), the above argument can be used also for holomorphic coverings of one domain $\Omega$ onto a subset $\hat{\Omega} \backslash \hat{E}$ of another domain $\hat{\Omega}$, where $\hat{E}$ is negligible. As an application, observe that the mapping $\left(z_{1}, z_{2}\right) \mapsto\left(z_{2}, e^{-z_{1} / 2}\right)$ is a holomorphic covering of the "tube" domain $\mathcal{D}_{0}$ from Example 5.4 onto the Springer domain (Example 3.20) with the axis $z_{2}=0$ removed. Composing with the Cayley transform, we see that the Springer domain with the axis removed is covered by the unit ball. From the validity of (5.11) on the ball, we can therefore deduce that (5.10) must hold for the induced metric, given by $\Psi=-\log a, a(x)=\left(-\left|x_{1}\right|^{2}-\log \left|x_{2}\right|^{2}\right)^{3}$, on the Springer domain. (This can also be checked by direct computation, using Lerch's inversion formula, in a similar way as in the last section of [EP].) We omit the details.

Thus, generally speaking, we still don't have any other example of a Kähler manifold satisfying (5.11) than $\Omega=\mathbf{C}^{n}$ and $\Omega$ a bounded symmetric domain. Is it true that every Kähler manifold for which (5.11) holds is subordinate to one of these two? 


\section{REFERENCES}

[BFG] M. Beals, C. Fefferman, and R. Grossman, Strictly pseudoconvex domains in $\mathbf{C}^{n}$, Bull. Amer. Math. Soc. 8 (1983), 125-326. MR 85a:32025

[Ber1] F.A. Berezin, Quantization, Math. USSR Izvestiya 8 (1974), 1109-1163. MR 52:16404

[Ber2] F.A. Berezin, Quantization in complex symmetric spaces, Math. USSR Izvestiya 9 (1975), 341-379. MR 58:22691

[BC1] C.A. Berger and L.A. Coburn, Toeplitz operators on the Segal-Bargmann space, Trans. Amer. Math. Soc. 301 (1987), 813-829. MR 88c:47044

[BC2] C.A. Berger and L.A. Coburn, Heat flow and Berezin-Toeplitz estimates, Amer. J. Math. 116 (1994), 563-590. MR 95g:47038

[BCZ] C.A. Berger, L.A. Coburn and K.H. Zhu, Function theory on Cartan domains and the Berezin-Toeplitz symbol calculus, Amer. J. Math. 110 (1988), 921-953. MR 89m:32053

[Berg] S. Bergman, The kernel function and conformal mapping, Math. Surveys, vol. 5, AMS, Providence, 1950. MR 12:402

[BM] S. Bochner, W.T. Martin, Several complex variables, Princeton, 1948. MR 10:366

[BLU] D. Borthwick, A. Lesniewski, H. Upmeier, Non-perturbative deformation quantization on Cartan domains, J. Funct. Anal. 113 (1993), 153-176. MR 94d:47065

[CGR] M. Cahen, S. Gutt, J. Rawnsley, Quantization of Kähler manifolds, I: Geometric interpretation of Berezin's quantization, J. Geom. Physics 7 (1990), 45-62; II, Trans. Amer. Math. Soc. 337 (1993), 73-98; III, Letters in Math. Phys. 30 (1994), 291-305. MR 92e:58082, 93i:58063; 95c:58082

[ChY] S.Y. Cheng, S.-T. Yau, On the existence of a complete Kähler-Einstein metric on noncompact complex manifolds and the regularity of Fefferman's equation, Comm. Pure Appl. Math. 33 (1980), 507-544. MR 82f:53074

[C1] L.A. Coburn, Berezin-Toeplitz quantization, in: Algebraic methods in Operator Theory, Birkhäuser, 1994, pp. 101-108. MR 95f: 47046

[C2] L.A. Coburn, Deformation estimates for the Berezin-Toeplitz quantization, Comm. Math. Phys. 149 (1992), 415-424. MR 93j:47038

[CX] L.A. Coburn, J. Xia, Toeplitz algebras and Rieffel deformations, Comm. Math. Phys. 168 (1995), 23-38.

[DK] D.M. DeTurck, J.L. Kazdan, Regularity theorems in geometry, Ann. Sci. École Norm. Sup. 14 (1981), 249-260. MR 83f:53018

[E1] M. Engliš, Density of algebras generated by Toeplitz operators on Bergman spaces, Arkiv för Matematik 30 (1992), 227-243. MR 95e:47036

[E2] M. Engliš, Asymptotics of reproducing kernels on a plane domain, Proceedings Amer. Math. Soc. 123 (1995), 3157-3160.

[E3] M. Engliš, Asymptotics of the Berezin transform and quantization on planar domains, Duke Math. J. 79 (1995), 57-76.

[EP] M. Engliš, J. Peetre, On the correspondence principle for the quantized annulus, Math. Scand. (to appear).

[FK1] J. Faraut, A. Korányi, Function spaces and reproducing kernels on bounded symmetric domains, J. Funct. Anal. 88 (1990), 64-89. MR 90m:32049

[FK2] J. Faraut, A. Korányi, Analysis on symmetric cones, Clarendon Press, Oxford, 1994.

[Helg] S. Helgason, Differential geometry and symmetric spaces, Academic Press, New York, 1962. MR 26:2986

[Hörm] L. Hörmander, An introduction to complex analysis in several variables, Van Nostrand, Princeton, 1966. MR 34:2933

[JP] M. Jarnicki, P. Pflug, Invariant distances and metrics in complex analysis, Walter de Gruyter, 1993. MR 94k:32039

[KL] S. Klimek, A. Lesniewski, Quantum Riemann surfaces, I: The unit disc, Comm. Math Phys. 146 (1992), 103-122; II: The discrete series, Letters in Math. Phys. 24 (1992), 125-139. MR 93g:58009; 93k:58011

[Kra] I. Kra, Automorphic functions and Kleinian groups, Benjamin, 1972. MR 50:10242

[Krn] S.G. Krantz, Convexity in complex analysis, in: Several complex variables and complex geometry, part 1 (E. Bedford, J.P. D’Angelo, R.E. Greene, S.G. Krantz, eds.), Proc. Symposia Pure Math., vol. 52, 1994, pp. 119-138. 
[LS] S.H. Liu, M. Stoll, Projections on spaces of holomorphic functions on certain domains in $\mathbf{C}^{2}$, Complex Variables 17 (1992), 223-233. MR 92m:32041

[M] N. Mok, Complete Kähler-Einstein metrics on bounded domains locally of finite volume at some boundary points, Math. Ann. 281 (1988), 23-30. MR 89m:32029

[MY] N. Mok, S.-T. Yau, Completeness of Kähler-Einstein metric on bounded domains and the characterization of domains of holomorphy by curvature conditions, Proc. Symposia in Pure Mathematics, vol. 39, part 1, 1983, pp. 41-59. MR 85j:53068

[Mor] C. Moreno, *-products on some Kähler manifolds, Letters Math. Phys. 11 (1986), 361372. MR 88c: 58021

[Pe1] J. Peetre, The Berezin transform and Ha-plitz operators, J. Operator Theory 24 (1990), 165-168. MR 91k:47058

[Pe2] J. Peetre, Correspondence principle for quantized annulus, Romanowski polynomials, Morse potential, J. Funct. Anal. 117 (1993), 377-400. MR 94m:47056

[Rie1] M.A. Rieffel, Quantization and $C^{*}$-algebras, Contemp. Math., vol. 167, AMS, 1994, pp. 66-97. MR 95h:46108

[Rie2] M.A. Rieffel, Deformation quantization for $\mathbf{R}^{d}$ actions, Memoirs Amer. Math. Soc. 106 (1993), no. 506. MR 94d:46072

[Skw] M. Skwarczynski, Biholomorphic invariants related to the Bergman function, Dissertationes Math. 173 (1980). MR 82e:32038

[Spr] G. Springer, Pseudo-conformal transformations onto circular domains, Duke Math. J. 18 (1951), 411 - 424. MR 12:817

[Su] T. Sunada, Holomorphic equivalence problem for bounded Reinhardt domains, Math. Ann. 235 (1978), 111-128. MR 58:1211

MÚ AV ČR, Žitná 25, 11567 PRAgue 1, CZECh Republic

E-mail address: englis@csearn.bitnet 